\title{
Simulating Pluto's Atmosphere with Hybrid Fluid/Kinetic Models
}

\author{
A dissertation \\ Presented to \\ the Faculty of the School of Engineering and Applied Science \\ University of Virginia
}

In Partial Fulfillment

of the requirements for the Degree

Doctor of Philosophy

by

Justin Erwin

May

2013 
(C) 2013 Justin Erwin 


\section{Abstract}

Recent and planned spacecraft exploration of the planets and moons of our solar system have greatly increased interest in atmospheric escape. The Cassini spacecraft is currently improving our understanding of the upper atmosphere of Saturn's large moon Titan, in 2014 the Maven spacecraft will begin to orbit Mars to study its upper atmosphere and atmospheric loss, and in 2015 the New Horizons spacecraft will have a close flyby encounter with Pluto. My motivation has been to produce an accurate model of Pluto's atmosphere, which includes atmospheric loss by escape. The results will be both predictive for and tested against data obtained during the New Horizon encounter. By accurately describing the present loss rates, one can hope to eventually be able to extrapolate back in time in order to describe the long-term evolution of Pluto's atmosphere. Doing this accurately for a planet for which we will have in situ spacecraft data will then guide our ability to model atmospheres for a large number of exoplanets observed orbiting other stars for which there is only remote sensing data.

Constraints on Pluto's atmosphere have been obtained through modeling and a few stellar occultation events in the last few decades. These have set a surface pressure range of 6.5-24 microbar of the primarily nitrogen atmosphere, with a methane mixing ratio of $\sim 0.5 \%$. Carbon monoxide has been detected as a trace species out to $\sim 4$ planetary radii, suggesting an atmosphere that is much more extended than predicted. Typically hydrodynamic models have been applied to describe escape from planetary bodies such as Pluto and Titan. Such models require solving the fluid equations out to very large distances from the planet in order to enforce boundary conditions. However, it is known 
through molecular kinetic simulations that at some finite distance above the surface the fluid equations fail to describe the gas properties accurately as the atmosphere transitions into the largely collisionless exosphere. To accurately capture the nature of the escape and structure of Pluto's thermosphere and exosphere, I have developed a model of Pluto's upper atmosphere by connecting a fluid model, using radiative heating models relevant for the thermosphere and stratosphere, to a molecular kinetic model. Using this hybrid model I have shown that the atmosphere is much more extended than previously predicted and the escape is not supersonic, as in comet or stellar atmospheres. Rather, it is closer to the evaporative Jeans model of escape.

Pluto's lower atmosphere or upper atmosphere have typically been modeled separately, without considering the interactions between these two regimes. Therefore, I have developed a self-consistent model of Pluto's full atmosphere, including both the stratospheric radiative heating and cooling that prevail in the lower atmosphere, as well as the UV heating and the cooling by atmospheric escape. These reach a sensitive balance in the upper atmosphere. The stratospheric processes included non-LTE IR radiative heating and cooling models for methane and carbon monoxide that have previously been used to describe Pluto's lower atmosphere and to constrain the surface conditions (i.e. pressure, mixing ratios, etc.) to fit observations. The resulting atmosphere is highly extended, with the exobase altitude and escape rate most dependent on the net UV heating. I find that adiabatic cooling due to the escaping atmosphere is important throughout the entire atmosphere, whereas it is usually ignored in the lower atmosphere where conduction and IR processes are the dominant heating/cooling mechanisms. Furthermore, the effects of the surface condition on the escape process and its evolution 
through Pluto's orbit and the Sun's solar cycle are considered. The results have been made available to the New Horizons team in preparation for atmospheric observations during its flyby in 2015. The results of my 1D surface to exosphere model will also be available to others to validate the assumptions of the highly complex 2D and 3D GCM models of Pluto's lower atmosphere. 


\section{APPROVAL SHEET}

The dissertation

is submitted in partial fulfillment of the requirements

for the degree of

Doctor of Philosophy

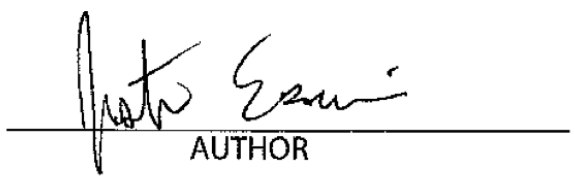

The dissertation has been read and approved by the examining committee:

\section{Advisor}

Accepted for the School of Engineering and Applied Science:

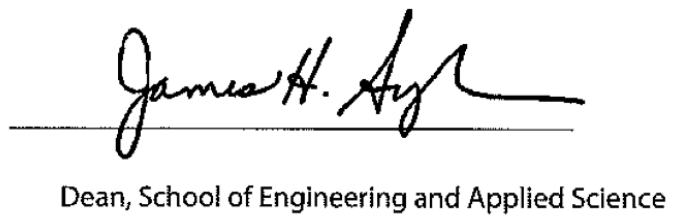

May

2013 


\section{CONTENTS}

CONTENTS

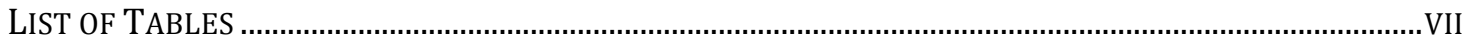

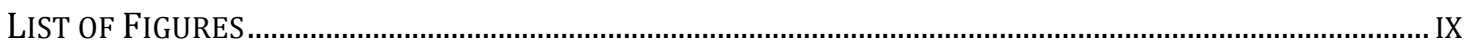

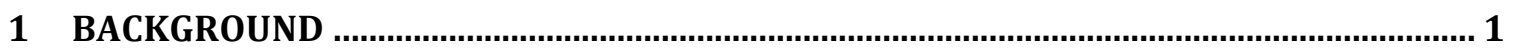

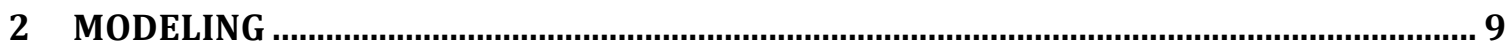

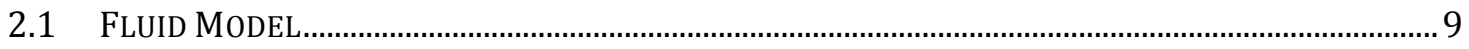

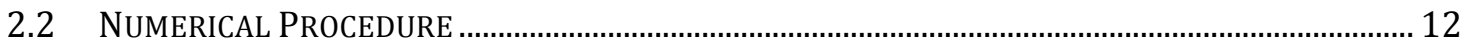

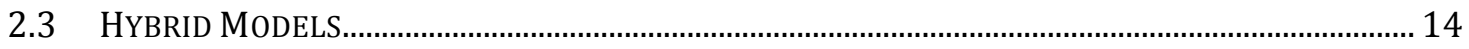

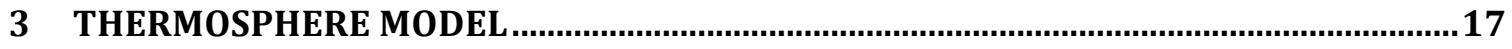

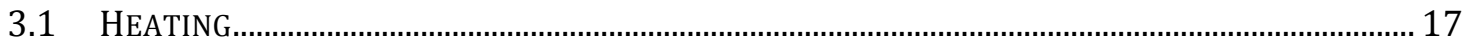

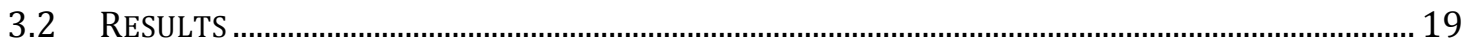

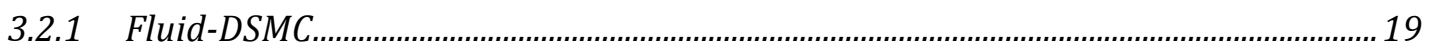

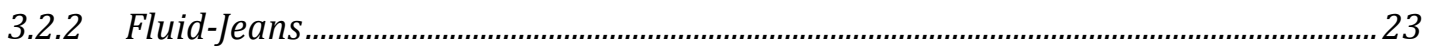

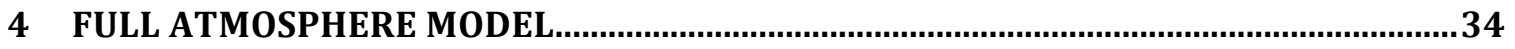

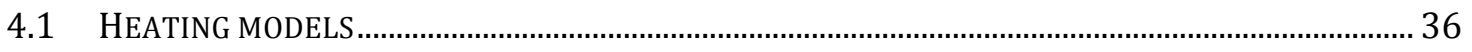

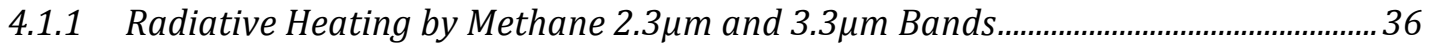

4.1.2 Non-LTE Radiative Cooling by Methane 7.6

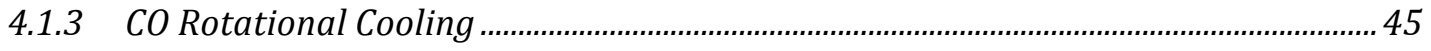

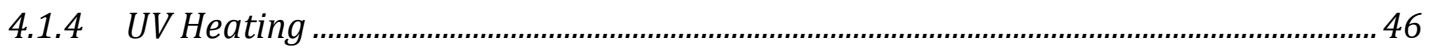

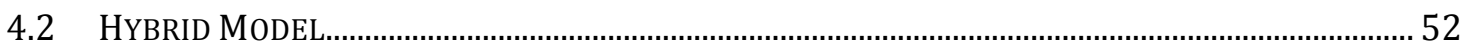

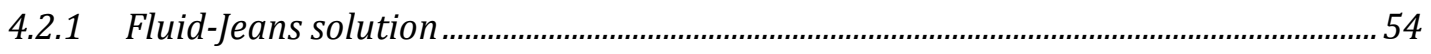

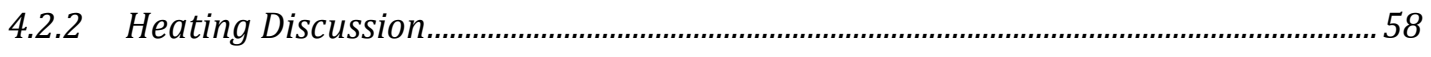


4.2.3 Fluid-DSMC Results........................................................................................................ 61

4.2.4 Surface Pressure, Mixing Ratio, and Orbital Distance................................................ 63

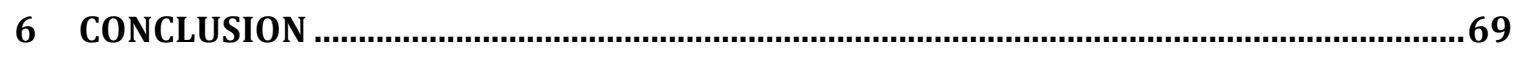

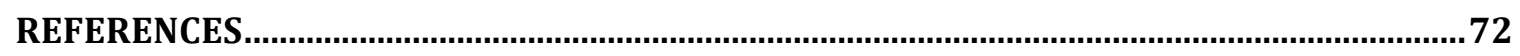

APPENDIX A: THERMALLY DRIVEN ATMOSPHERIC ESCAPE: TRANSITION FROM

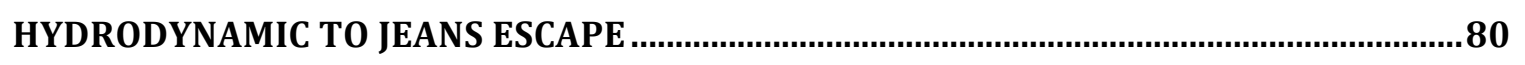

APPENDIX B: THERMALLY DRIVEN ESCAPE FROM PLUTO'S ATMOSPHERE: A

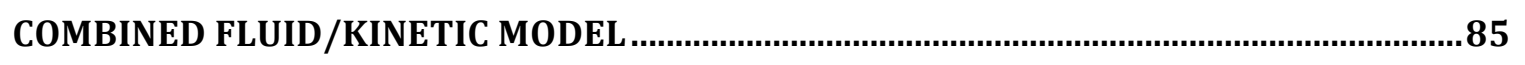

APPENDIX C: MOLECULAR-KINETIC SIMULATIONS OF ESCAPE FROM THE EX-

PLANET AND EXOPLANETS: CRITERION FOR TRANSONIC FLOW …................................ 93 


\section{List of Tables}

Table 1: Fluid-DSMC results. $E_{\mathrm{esc}}=\phi_{\mathrm{E}} / \phi$ is the average energy carried off per molecule; $r_{x}$ is given as a ratio to $r_{p}=1150 \mathrm{~km} . \phi_{\mathrm{J}}$ is computed using exobase values from the solution, $\phi_{\mathrm{L}}$ is computed using $\mathrm{Q}$ from the solution and $\mathrm{r}=1450$ $\mathrm{km}$.

Table 2: Results of the Fluid-Jeans model. The first results uses Jeans escape rate values for $\phi$ and $\phi_{\mathrm{E}}$, while the second set uses twice the Jeans escape rate values. The exobase radius, $r_{x}$, is given a ratio relative to $r_{p}=1150 \mathrm{~km}$ 25

Table 3: Fluid-Jeans model results for solar maximum case. Results are given for $1 \times$, $2 \times$, and $3 \times$ the Jeans escape values as an upper boundary condition. The last column ( $3 \times$ Jeans $)$ is an estimate of the enhancement, inferred from the FluidDSMC results in Table 1

Table 4: (Left) Escape rate $\phi$ (solid line) plotted versus net heat deposited Q from the Fluid-Jeans model. Overlaid are escape rates from the Fluid-DSMC model as black diamonds, and from the SHE model (Strobel, 2008) as x's. Also plotted is the energy limited escape rate $\phi_{\mathrm{L}}=\mathrm{Q} / \mathrm{U}\left(\mathrm{r}_{0}\right)$ (dashed line). (Right) Comparison energy loss mechanisms versus net heat deposited from the Fluid-Jeans model

Table 5: Comparing the Fluid-Jeans model, Hunten and Watson (1982), McNutt (1989) and energy-limited escape (ELE). For each model, the values for each given are the escape rate $\phi$, the location of the heating layer in terms of the lower boundary $r_{h} / r_{0}$ (in the case of the Fluid-Jeans model I give the location of the peak heating, which is where the slant optical depth is unity), and the net heating $Q$ are shown. The ELE 
model was applied in 4 ways: (a) using $\mathrm{Q}$ from the Fluid-Jeans model, (b) $\mathrm{r}_{\mathrm{h}}$ is altitude of unit parallel optical depth, (c) $r_{h}$ is the altitude of unit vertical optical

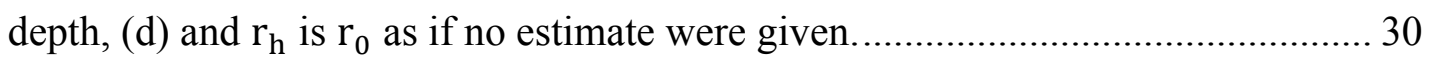

Table 6: UV and EUV absorption cross section and energy flux values from

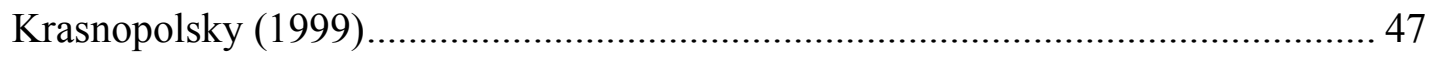

Table 7: Effective cross section and effective energy fluxes computed from the SSI for March 30, 2003 (a solar minimum like case) scaled to $30 \mathrm{AU}$ for the sub-ranges 0$80 \mathrm{~nm}$ and $80-140 \mathrm{~nm}$. 50

Table 8: Values for effective cross section and effective solar energy flux given in the 3 sub-ranges for case of solar minimum, mean, and maximum-like conditions. 51

Table 9: Base case parameters for full atmosphere model. 52

Table 10: Values from the solutions from the fluid-Jean full atmospheric model. 56

Table 11: Comparison of energy loss mechanism through upper and lower boundaries. 57

Table 12: The integrated heating rates from the different heating mechanisms. 58

Table 13: Characteristic values from fluid-DSMC simulation of Pluto's full atmosphere. 62 


\section{List of Figures}

Figure 3-1: Temperature (right) and density (left) profiles for the hybrid Fluid-DSMC model of Pluto, for the cases of zero heating and solar minimum (from Tucker et al., 2012), and our new result for solar mean. The temperate profiles are plotted with the scale labeled below; the density profiles are plotted on a logarithmic scale, with the scale labeled above. The exobase altitudes are marked for each case by $r_{x}$. The DSMC solutions are time-averaged after reaching steady state to smooth out random oscillations.

Figure 3-2: Temperature (right) and density (left) profiles for solar mean conditions, comparing the Fluid-DSMC solution of the present study with that of the SHE from Strobel (2008), along with the Fluid-Jeans. Marked on the Fluid-DSMC solution is the exobase $\left(r_{\mathrm{x}}\right)$ as well as the transition between the fluid model and DSMC $\left(r_{t}\right.$, where $\left.\mathrm{Kn}_{\mathrm{rt}}=0.1\right)$. Marked on the SHE model are the exobase $\left(\mathrm{r}_{\mathrm{x}}\right)$ and the sonic point $\left(\mathrm{r}_{\mathrm{s}}\right)$. 20

Figure 3-3: Temperature profile found in the Fluid-Jeans model for the 4 special cases discussed: zero heating, solar minimum, solar mean, and solar maximum. The top of each curve represents the exobase altitude.

Figure 4-1: Absorption cross-section for nitrogen and methane from 0 to $140 \mathrm{~nm}$. Solar spectral irradiance for the same range from March 30, 1999 retrieved from http://lasp.colorado.edu/lisird/see/. 48 
Figure 4-2: Conductivity for nitrogen for a temperature range relevant to Pluto. Stevens et al. (1992), Hubbard et al. (1990), and Bird (1994) are power laws, while Lemmon et al. (2004) is a detailed parameterization.

Figure 4-3: Temperature profiles of solutions to fluid-Jeans full atmosphere model....... 55

Figure 4-4: Density profile for Fluid-Jeans solutions. 55

Figure 4-5: Heating and cooling rates in the lower atmosphere for the fluid-Jeans solution with solar minimum UV activity. By the sign convention for the cooling rates, a solid line denotes local cooling and a dotted denotes local heating.

Figure 4-6: Heating and cooling rates for the fluid-Jeans solution with solar minimum UV activity. By the sign convention for the cooling rates, a solid line denotes local cooling and a dotted denotes local heating. 60

Figure 4-7: Temperature profiles from fluid-DSMC model of Pluto's full atmosphere. . 62

Figure 4-8: Temperature profiles in the lower atmosphere for surface pressures between 0.8 and 2.2Pa. 64

Figure 4-9: Density profiles in the lower atmosphere found by varying the methane mixing ratio. Also plotted as a dotted line is the best-fit solution from Zalucha et al. (2011) to the Siding Spring stellar occultation. The surface pressure was set to 1.32Pa 65

Figure 4-10: Temperature solutions for carbon monoxide mixing ratio between 0.0 and $0.1 \%$. 66

Figure 4-11: Integrated heating rates through Pluto's eccentric orbit. The solar UV activity was fixed to the solar minimum case. 


\section{Background}

Recent spacecraft exploration of the planets and moons in our solar system and the rapid increase in the discovery of exoplanets has increased interest in atmospheric escape from planetary bodies. The Cassini spacecraft is currently improving our understanding of atmospheric escape from Titan, while in 2015 the New Horizons (NH) spacecraft will flyby Pluto and perform occultation observations of its and Charon's atmosphere (Guo and Farquhar, 2005), and the Maven mission will orbit Mars studying the composition of its escaping atmosphere (Lin and Jakosky, 2012). Furthermore, newly discovered exoplanets, with radii ranging from about Earth to a few times Jupiter, and their atmospheres are also modeled (Yelle, 2004; Lammer et al., 2009; Murray-Clay et al., 2009). Here I carry out simulations of Pluto's upper atmosphere, including atmospheric loss by thermal escape, that can be tested against data to be obtained during the $\mathrm{NH}$ encounter. By accurately describing the present loss rates, one can in principle learn about the evolution of Pluto's atmosphere. In addition, doing this accurately for a planet for which I will have in situ spacecraft data can, by scaling, guide our ability to model exoplanet atmospheres for which there will only be remote sensing data.

Although discovered in 1930, it wasn't until the 1980's that the existence of Pluto's atmosphere was confirmed. NASA's Kuiper Airborne Observatory gave the first definitive evidence of the atmosphere in 1988 when the atmosphere caused a gradual dimming in the light curve as Pluto transited in front of a star, referred to as a stellar occultation (Elliot et al., 1989). From these light curves and some assumptions of its 
atmospheric composition, it was determined that Pluto has a temperature inversion near its surface caused by radiative absorption of methane (Yelle et al., 1989). Its surface temperature is near $40 \mathrm{~K}$, but the temperature of its atmosphere increases with altitude to about $100 \mathrm{~K}$.

Due to Pluto's low gravity and large distance from the Sun, its atmosphere is quite different from those of the terrestrial planets. However, Pluto is large enough to retain an atmosphere over planetary time scales. The most similar known object in our solar system is Triton, a moon of Neptune. Triton is comparable in size to Pluto and has a primarily nitrogen atmosphere with methane, carbon monoxide and carbon dioxide (Cruikshank et al., 1993). Many of the observational and modeling techniques initially used on Triton, and validated against the Voyager 2 flyby, were subsequently applied to Pluto (Owen et al., 1993; Strobel and Xhu, 1996; Krasnopolsky, 1999).

Spectral measurements have been used to determine the composition of Pluto's surface and atmosphere. Owen (1993) detected the presence of carbon monoxide and nitrogen ices, and confirmed the presence of methane ice in the 1.4-2.4 micron region. That study also determined that nitrogen must be more abundant on the surface by at least a factor of 50, and therefore must dominate the atmospheric composition. Lellouch et al. (2011) used high-resolution spectrometry to further constrain the relative abundance of these atmospheric gases.

The temperature and density structure of Pluto's lower atmosphere has been fit to the light curves of stellar occultation. Following the initial detection (Elliot et al., 1989), numerous subsequent observations have expanded our knowledge of the temporal and geographic variations of the atmosphere (Sicardy et al., 2003; Young et al., 2008; Young 
et al., 2010). Unlike Triton, Owen et al. (1993) found no evidence for carbon dioxide in Pluto's atmosphere. Lellouch et al. (2009) used both spectroscopy and stellar occultation to constrain the surface pressure to between 6.5 and 24 microbar, and found that a methane mixing ratio of $0.5 \%$ was adequate to explain the inverted temperature structure and the $\sim 100 \mathrm{~K}$ temperature in the upper atmosphere. Lellouch et al. (2011) recently refined their methane mixing ratio and determined a carbon monoxide mixing ratio of $0.05 \%$. Greaves et al. (2011) reported carbon monoxide emission out to 3 planetary radii above the surface, suggesting a significantly extended atmosphere consistent with our preliminary modeling (Tucker et al., 2011)

Strobel et al. (1996) developed a radiative-conductive model of Pluto's lower atmosphere, balancing radiative absorption and emissions by methane and carbon monoxide with conductive heat transport. The resulting temperature structure compared well with those inferred from stellar occultation. Zalucha et al. (2011) used this radiative conductive model to constrain the free parameters (surface pressure, radius, temperature and mixing ratios) by fitting to stellar occultations. They found similar values to those given in Lellouch et al. (2009) and Lellouch et al. (2011). Zalucha et al. (2012) recently used a global circulation model of the lower atmosphere, combined with a simplified radiative model, to investigate the wind structure.

The previous models of atmospheric escape for Pluto used the concept of hydrodynamic escape by adapting the critical solution described by Parker (1964). In that paper Parker described the expanding stellar corona and stellar wind assuming the temperature and pressure go to zero at infinity, and showed that for this to happen the 
bulk velocity must increase past the isothermal speed of sound at a critical point that is dependent on the temperature and gravity.

This model was subsequently adapted for planetary atmospheres to include heating by solar radiation (Hunten and Watson, 1982; McNutt, 1989) and was applied to Pluto (Krasnopolsky, 1999; Strobel, 2008) and Titan (Strobel, 2009). It is often referred to as the slow hydrodynamic escape (SHE) model. The model requires solving the fluid equations out to very large distances from the planet to enforce the necessary boundary conditions. However, it is known that at some finite distance from the planetary surface the equations of fluid dynamics fail to describe the flow of mass, momentum and energy in an atmosphere (Johnson, 2010, Volkov et al., 2011). The region of validity of the fluid equation is often defined using the Knudsen number, $K n=\ell / H$, the ratio of the mean free path of the molecules, $\ell=(\sqrt{2} \sigma n)^{-1}$, to the density scale height, $H=\frac{k_{b} T}{m g(r)}$, of the atmosphere, where $\sigma$ is the collisional cross-section of the $\mathrm{N}_{2}$ molecule, $n$ the local number density, $k_{b}$ the Boltzmann constant, $T$ the temperature, $m$ the mass of an $\mathrm{N}_{2}$ molecule, and $g(r)=\frac{G M}{r^{2}}$ the local gravitational acceleration. The fluid equations properly capture the physics in region where $K n \ll 1$ so that many collisions occur over relevant length scales keeping the gas in local thermal equilibrium.

The alternative to organized outflow is evaporative escape, by which the atmosphere loses gas on a molecule-by-molecule basis driven by conductive heat flow from below. The standard analytic model was originally developed by Jeans (1925) and is referred to as Jeans escape. The escape rates are found to depend predominately on the Jeans parameter $\lambda=\frac{U(r)}{k_{b} T}$, the ratio of gravitational, $U(r)=\frac{G M m}{r}$, to thermal energy of 
the molecules. For large Jeans parameters (i.e. when gravitational energy dominates thermal energy) the escape rate is obtained by integrating the velocity distribution over the portion of molecules that are moving upward with speed in excess of the escape velocity. Assuming a Maxwell-Boltzmann velocity distribution, the molecular escape rate $\phi$ and energy escape rate $\phi_{E}$ from an altitude $r$ are given by

$$
\begin{gathered}
\langle\phi\rangle_{J}=\frac{1}{4} n \bar{v}(1+\lambda) \exp (-\lambda) \\
\left\langle\phi_{E}\right\rangle_{J}=\langle\phi\rangle_{J}\left(\frac{C_{p}}{k_{b}}-\frac{3}{2}+\frac{1}{1+\lambda}\right) k_{b} T
\end{gathered}
$$

Here $\bar{v}=\sqrt{8 k_{b} T / m \pi}$ is the mean molecular speed, and $C_{p}=\frac{7}{2} k_{b}$ is the specific heat at constant pressure per molecule. To be consistent with the fluid equations, I also consider expressions with a drifting Maxwell-Boltzmann to include the bulk velocity $u$ in the velocity distribution (Yelle, 2004; Tian et al., 2009; Volkov et al., 2011). Typically these equations are applied at a level called the exobase where $K n \sim 1$, as there are few collisions above this level to inhibit a molecule from escaping.

Chassefière (1996), Yelle (2004) and Tian (2008) have used Jeans escape as a boundary condition in place of the hydrodynamic escape boundary condition in modeling planetary atmospheres that have significant escape rates. In modeling the response of the Earth's thermosphere to EUV heating, Tian used the Jeans escape expression to determine the velocity at the upper boundary (the exobase in their case); however at the upper boundary a zero temperature gradient was applied to neutrals, and a fixed heat gradient was applied to electrons separately. In modeling extra-solar gas giants at small orbital distance, Yelle (2004) also used Jeans escape, with a drifting Maxwell-Boltzmann 
velocity distribution, to get the gas flow velocity at his upper boundary. However, this was applied at a fixed upper boundary of 3 planetary radii rather than at the exobase.

To capture the non-equilibrium nature of rarified flow, Bird $(1963 ; 1994)$ and others developed Monte Carlo models for describing the molecular behavior of the gas. These have been shown to correctly represent solutions to the Boltzmann equations. One uses representative particles along with a collisional cross section model to simulate the behavior of the gas including its flows. The method of Bird is called the Direct Simulated Monte Carlo (DSMC) model in which the particles move along physical paths while collisions are treated as a random process. Density, temperature, and bulk velocity of the gas are recovered by averaging over the positions and velocities of the representative molecules. This model has been applied to many instances of rarified flow, including the expanding gas from a comet (Tenishev et al., 2008), the tenuous atmospheres of moons (Walker et al., 2010), and the spreading of a neutral torus around planets (Cassidy et al., 2010). Tucker et al. (2009; 2012) used this method to model escape from Titan.

Such modeling is an integral part of the work that will constitute my thesis as described below. To describe the gas, representative particles with weights are tracked in the gravitational field of the planet subject to mutual collisions. This results in a density and representative velocity distribution function versus distance from the planet, from which the properties of the atmosphere can be calculated at each altitude. Such a model can in principle describe the behavior of a gas at any density, but in practice, as the atmospheric density varies significantly over altitude, its application is limited by numbers of representative molecules that can be tracked, so is most efficiently used to describe the region in the vicinity of the exobase. 
Using the DMSC method Volkov et al. (2011) demonstrated earlier that for a monatomic or diatomic gas in the absence of heating above some lower boundary for $\lambda \gtrsim 3$ the escape is Jeans-like at the exobase (i.e. $\phi /\langle\phi\rangle_{J} \sim 1.5$ for the range of Jeans parameters studied). For our model of Pluto's upper atmosphere, the lower boundary of the simulated domain has $\lambda=22.8$, and the calculated exobase values exceed 4. Using the above results as guidance, I model the principal component in Pluto's atmosphere to obtain a description relevant to the $\mathrm{NH}$ encounter. I did this in order to test energy-limited escape, and to better understand the transition from Jeans to hydrodynamic escape.

The concept of energy-limited escape, which I will examine here, is heavily used in modeling escape from early terrestrial planet atmospheres (Tian et al., 2009) and the growing body of data on exoplanet atmospheres (Lammer et al., 2009; Valencia et al., 2010). EUV radiation, assuming that kinetic and thermal energy terms are small compared to gravity, and the thermal conduction is inefficient, the molecular loss rate is often approximated as

$$
\phi_{L} \simeq \frac{Q}{U(r)}
$$

Here $Q$ is the EUV energy supplied in the upper atmosphere, and $U(r)$ is the gravitational potential energy. Without doing a detailed heating model one can use $Q=\pi r_{E U V}^{2} \varepsilon_{U V} F_{E U V}$, where $r_{E U V}$ is the mean radius at which the radiation is absorbed and $\varepsilon_{U V}$ and $F_{E U V}$ are the heating efficiency and solar energy flux respectively. The estimated escape rate depends critically on $r_{E U V}$, which is sometimes assumed to be close to the visual radius so $r_{E U V}=r_{P}$. Also important is that atmospheric radius from which 
the molecules being removed from the gravitational well are being re-supplied; again the visual radius is often chosen so that $U\left(r_{p}\right)$ is used. These aspects will be examined.

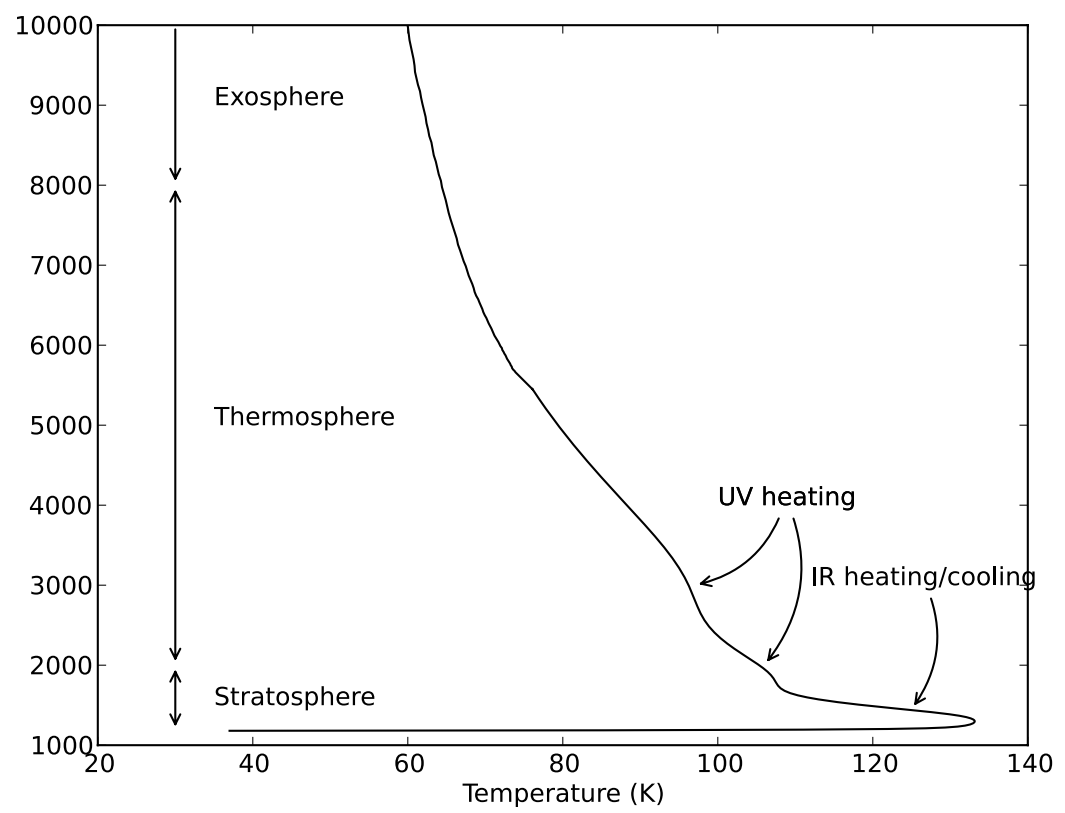

Figure 1-1: The full description of Pluto's atmosphere using the fluid-DSMC model.

Finally, I will develop a model of Pluto's full atmosphere from the surface to well past the exobase using the hybrid fluid-DSMC model that will be introduced. I include the Strobel et al. (1996) model of non-LTE IR heating and cooling of methane and carbon monoxide important in the stratosphere. UV heating by nitrogen and methane included in the thermosphere is shown to be the source of energy for the escaping molecules. The DSMC model describes the upper thermosphere and exosphere where the atmosphere changes from being collisional to near free molecular flow. 


\section{Modeling}

\subsection{Fluid Model}

The steady state equations of mass, momentum, and energy have been used to study hydrodynamic escape. They can be solved to give the radial dependence of number density $n$, outward bulk velocity $u$, and temperature $T$ in the region in which the atmosphere is collisional. However, as we have shown (Tucker et al., 2012; Volkov et al., 2011) they cannot be used by themselves to determine the escape rate unless the Jeans parameter is very small in which case the solutions are somewhat insensitive to the boundary conditions at infinity. The equations, neglecting viscosity can be written as

$$
\begin{gathered}
4 \pi r^{2} n u=\phi \\
n m \frac{d\left(\frac{1}{2} u^{2}\right)}{d r}+\frac{d p}{d r}=-n m g(r) \\
\frac{d}{d r}\left(\phi\left(C_{p} T+\frac{1}{2} m u^{2}-U(r)\right)-4 \pi r^{2} \kappa(T) \frac{d T}{d r}\right)=4 \pi r^{2} q(r)
\end{gathered}
$$

Here $\phi$ is the molecular escape rate through the one dimensional atmosphere, $p$ is the pressure (related via the equation of state, $p=n k_{b} T$ ), $m$ is the mass of a $\mathrm{N}_{2}$ molecule,

$\kappa(T)$ is the conductivity, $C_{p}=\frac{7}{2} k_{b}$ is the specific heat at constant pressure, and $q(r)$ is the net heating/cooling rate per unit volume. In Eq. (2.3), the first term on the left side is the work done by adiabatic expansion and the second is the divergence of the thermal conduction flux. 
For the conductivity, I use the power law $\kappa(T)=\kappa_{0} T^{\omega}$ to approximate the temperature dependence. Some authors use an empirical fit for the conductivity, e.g. Hunten and Watson (1982) used $\omega=1.12$, while McNutt (1989) used $\omega=1$ since it simplifies the analytic solutions to Eq. (2.3) In this chapter I use $\kappa_{0}=9.37 \times 10^{-5} \mathrm{~J} \mathrm{~m}^{-1}$ $\mathrm{K}^{-1}$ and $\omega=1$ to compare with Strobel (2008) and because this is consistent with the variable hard sphere model for collisions between $\mathrm{N}_{2}$ molecules used in the DSMC model of the exosphere (Tucker et al., 2012; Volkov et al., 2011).

The lower boundary of our fluid domain is set at $1450 \mathrm{~km}$, consistent with the occultation results and the assumptions of Strobel (2008). To determine an escape rate and enforce the upper boundary condition, the exobase needs to be within the simulation domain. Since we showed earlier (Tucker et al., 2012) that the atmosphere is highly extended, the upper boundary is set to $16000 \mathrm{~km}$ to accommodate the solar maximum case. The radial grid requires fine spatial resolution for the first $3000 \mathrm{~km}$ where the bulk of the heating occurs; however, a coarse grid is sufficient above this. Therefore, I use a grid that is equally spaced in $r^{-1}$. With $N_{r}=1600$ radial grid points in $r$, the lower boundary radial step size, $\Delta r$, of order $1 \mathrm{~km}$ and the upper boundary $\Delta r$ of order $100 \mathrm{~km}$.

Previously, I directly solved the steady state Eqs. (2.1-2.3) by iteratively solving them along with the heating and the DSMC escape simulations until a consistent solution was found (Tucker et al., 2012). Here I reintroduce the time-dependence into the energy equation and iterate through time until convergence is achieved. This is found to be more numerically stable for the boundary conditions and the higher heating cases that I will consider here. I make the substitution $\xi=T^{\omega+1}$ as in (Strobel et al., 1996; Zalucha et al., 2011) in the time-dependent form of Eq. (2.3) to obtain a linear time-dependent PDE: 


$$
\begin{aligned}
\frac{d T}{d t}=\frac{(\omega+1) T^{\omega}}{n C_{p}} & \left(q(r)-\frac{\phi}{4 \pi r^{2}} \frac{d}{d r}\left(\frac{1}{2} m u^{2}-U(r)\right)\right)-\left(\frac{2 \kappa_{0} T^{\omega}}{n r C_{p}}-\frac{\phi}{4 \pi r^{2} n}\right) \frac{d \xi}{d r} \\
+ & \frac{\kappa_{0} T^{\omega}}{n C_{p}} \frac{d^{2} \xi}{d r^{2}}
\end{aligned}
$$

This equation is solved using the implicit, finite difference time stepping scheme described in Section (2.3). This second-order equation needs two boundary conditions. The first is the fixed lower boundary temperature $T\left(r_{0}\right)=88.2 \mathrm{~K}$, and the second is a restriction on the energy flux leaving the atmosphere from the top boundary $r_{t}$

$$
\left.4 \pi r^{2} \frac{\kappa_{0}}{\omega+1} \frac{d \xi}{d r}\right|_{r_{t}}=\phi\left(C_{p} T+\frac{1}{2} m u^{2}-U(\mathrm{r})\right)_{r_{t}}-\phi_{E}
$$

This condition is derived by integrating Eq. (2.3) from the exobase to the upper boundary and using $\phi_{E}$ for the energy flux from the atmosphere. This quantity is determined by the escape model, the details of which will be explained in Section (2.3)

The time stepping advances the temperature alone. To update density and bulk velocity, I first update the values of $u$ using Eq. (2.1) and the previous values of $n$. The new values of $u$ are then used to update $n$ using the momentum equation (2.2) reexpressed as

$$
p(r)=p_{0} \exp \left(\int_{r_{0}}^{r} \frac{\frac{1}{2} m \frac{d\left(u^{2}\right)}{d r}+m g(r)}{k_{b} T\left(r^{\prime}\right)} d r^{\prime}\right)
$$

Since Eqs. (2.1) and (2.6) are coupled through $n$ and $u$, I iteratively solve them together to find a consistent solution. A few iterations are sufficient to converge on a selfconsistent solution for $T, n$, and $u$. With the profiles updated, the last action of the time step is to recalculate the heating rates. 


\subsection{Numerical Procedure}

Here I describe the numerical equations and techniques used to solve the thermal diffusion equation on an unequally spaced grid. I define a grid $r_{m}$, where $m=1, \ldots, M$, the grid spacing $\Delta r_{m}=r_{m+1}-r_{m}$, the average grid spacing at $r_{m}$ as $\overline{\Delta r_{m}}=\left(r_{m+1}-\right.$ $\left.r_{m-1}\right) / 2$, and the finite difference approximation to $\xi\left(r_{m}\right)$ as $\xi_{m}$.

First I describe approximate the derivatives $\xi^{\prime}(r)$ and $\xi^{\prime \prime}(r)$ using the unequally spaced grid. Using the points $\xi_{m+1}, \xi_{m}, \xi_{m-1}$ to approximate $\xi^{\prime}(r)$, one might use a central difference scheme $\xi^{\prime}(r) \sim\left(\xi_{m+1}-\xi_{m-1}\right) /\left(2 \overline{\Delta r_{m}}\right)$; however, this scheme is not second order accurate, $O\left({\overline{\Delta r_{m}}}^{2}\right)$, as it would be in the case of equal spacing. The error term is proportional to $\left(\Delta r_{m}-\Delta r_{m-1}\right) \xi "(\tilde{r})$. While the quantity $\left(\Delta r_{m}-\Delta r_{m-1}\right)$ might be small compared to ${\overline{\Delta r_{m}}}^{2}$, the effect of this error in the model is to overestimate the conductivity. To correct for this I use the finite differences:

$$
\begin{aligned}
\xi^{\prime}(r) & =\frac{\Delta r_{m-1}^{2}\left(\xi_{m+1}-\xi_{m}\right)+\Delta r_{m}^{2}\left(\xi_{m}-\xi_{m-1}\right)}{2 \Delta r_{m} \Delta r_{m-1} \overline{\Delta r_{m}}} \\
\xi^{\prime \prime}(r) & =\frac{\Delta r_{m-1}\left(\xi_{m+1}-\xi_{m}\right)-\Delta r_{m}^{2}\left(\xi_{m}-\xi_{m-1}\right)}{\Delta r_{m} \Delta r_{m-1} \overline{\Delta r_{m}}}
\end{aligned}
$$

The error term on $\xi^{\prime}(r)$ is $\Delta r_{m} \Delta r_{m-1} \xi^{\prime \prime \prime}(\tilde{r})$, which is second order accurate and does not affect the conductivity. The error term for $\xi^{\prime}(r)$ is $\left(\Delta r_{m}-\Delta r_{m-1}\right) \xi^{\prime \prime \prime}(\tilde{r})$. While the finite difference approximation for $\xi^{\prime \prime}$ is not second order accurate, this is the minimal error term one can get from the 3 points used. It is important that I capture conduction correctly as to ensure a constant energy flux through the upper domain where there is zero heating and the grid spacing is large. 
I adapt the implicit scheme proposed by (Zalucha et al., 2011), using the same notation, to solve the diffusion equation

$$
\frac{d \xi}{d t}=A+B \xi+C \frac{d \xi}{d r}+G \frac{d^{2} \xi}{d r^{2}}+S
$$

on a domain $r \in\left(r_{0}, r_{t}\right)$. The coefficients $A, B, C, G$ and source term $S$ are functions of $r$ and $t$. The boundary conditions for the Fluid-Jeans model can be expressed as

$$
\begin{aligned}
& \xi\left(r_{0}\right)=\xi_{0} \\
& \left.\frac{d \xi}{d r}\right|_{r_{t}}=F_{t}
\end{aligned}
$$

I make two modifications to Zalucha's scheme. First, the finite difference expressions for an unequally spaced grid defined in Eqs. (2.7) and (2.8) are substituted into (2.9), and second, the finite difference formulation at the upper boundary will satisfy the energy flow restriction of Eq. (2.11) as well as the diffusion equation (2.9).

Implementing the time grid $t_{i}=i \Delta t$ for $i=0,1, \ldots$ and the notation $\xi\left(r_{m}, t_{i}\right)=\xi_{m}^{i}$. An implicit scheme is obtained by substituting the finite differences in Eqs. (2.7) and (2.8), evaluated at the advanced time step, into the diffusion equation in Eq. (2.9). For the time derivative, I use $\left(\xi_{m}^{i+1}-\xi_{m}^{i}\right) / \Delta t$. Solving for the advanced time step then requires solving a tridiagonal system of equations

$$
\alpha_{m} \xi_{m-1}^{i+1}+\lambda_{m} \xi_{m}^{i+1}+\omega_{m} \xi_{m+1}^{i+1}=q_{m}
$$

The lower boundary condition $\xi_{1}^{i}=\xi_{0}$ is then expressed as $\alpha_{1}=0, \lambda_{1}=1, \omega_{1}=0$, $q_{1}=\xi_{0}$. The interior point $(m=2, \ldots, M-1)$ are expressed as 


$$
\begin{gathered}
\alpha_{m}=\frac{\Delta t}{\Delta r_{m-1} \overline{\Delta r_{m}}}\left(\frac{\Delta r_{m}}{2} C_{m}-G_{m}\right) \\
\lambda_{m}=1-\Delta t\left(B_{m}+\frac{1}{\Delta r_{m-1} \Delta r_{m}}\left(\left(\Delta r_{m}-\Delta r_{m-1}\right) C_{m}-2 G_{m}\right)\right) \\
\omega_{m}=\frac{-\Delta t}{\Delta r_{m} \overline{\Delta r_{m}}}\left(\frac{\Delta r_{m-1}}{2} C_{m}+G_{m}\right) \\
q_{m}=\xi_{m}^{i}+\Delta t S_{m}
\end{gathered}
$$

The procedure for interior points is modified to enforce the condition on $\frac{d \xi}{d r}$ at the upper boundary. Eq. (2.11) is substituted directly into Eq. (2.9) for $\xi^{\prime}$. Using Eq. (2.8) with $m=M$ for $\xi^{\prime \prime}(r)$ at the upper boundary introduces the phantom point $\xi_{M+1}$, but by equating Eq. (2.7) to Eq. (2.11) I can remove it from the equation. The result is expressed as

$$
\begin{gathered}
\alpha_{M}=-\Delta t \frac{1+\Delta r_{M} / \Delta r_{M-1}}{\Delta r_{M-1} \overline{\Delta r_{M}}} \\
\lambda_{M}=1-\Delta t\left(B_{M}+\frac{1+\Delta r_{M} / \Delta r_{M-1}}{\Delta r_{M-1} \overline{\Delta r_{M}}} G_{M}\right) \\
\omega_{M}=0 \\
q_{M}=\xi_{m}^{i}+\Delta t\left(S_{m}+F_{t}\left(C_{M}+\frac{2}{\Delta r_{M-1}} G_{M}\right)\right)
\end{gathered}
$$

\subsection{Hybrid Models}

The hybrid model described here entails finding a fluid model that is consistent with a kinetic model of escape, as opposed to homogeneous boundary condition at infinity as in the usual hydrodynamic models. The two models are coupled since the kinetic model determines the escape rate $\phi$ and energy escape rate $\phi_{E}$, but depends on the solution of the fluid model. While the temperature found through Eq. (2.4) depends on $\phi$, the upper boundary conditions in Eq. (2.5) depend on both $\phi$ and $\phi_{E}$. Furthermore, the density and bulk velocity found through the coupled Eqs. (2.1) and (2.6) depend on $\phi$ and the 
temperature structure. In order to obtain $\phi$ and $\phi_{E}$, I use two models of escape in my hybrid simulations.

(i) The first hybrid model I use is to combine the fluid model of the thermosphere with a DSMC model of the exosphere as was done in Tucker et al. (2012). This I refer to as the Fluid-DSMC model. I refer readers to Tucker et al. (2012) for the details of the DSMC model used here, with the only change from that paper is my use of the time stepping method in the fluid model instead of the steady state fluid solution. This has been found to converge better in the presence of high heating, giving us the ability to find a FluidDSMC solution for solar mean conditions as described in the results section below.

The fluid equations and the DSMC simulation are coupled as the fluid equations depend on the escape rate $\phi$ and energy escape rate $\phi_{E}$ obtained from the DSMC simulation, while the DSMC simulation depends on the calculated temperature $T$, density $n$, and location $r$ of its lower boundary. The Fluid-DSMC solution is the self-consistent solution obtained through iteration.

I begin with an estimate of $\phi$ and $\phi_{E}$, and time step the fluid equation until convergence is obtained giving a first estimate of the fluid solution. I locate that $\mathrm{r}$ where $K n=0.1$ occurs in the fluid solution, and use the values of $T$ and $n$ at that $r$ to begin a DSMC simulation. This value of $K n$ is far enough below the exobase, in the collision-dominated regime, to act as a lower boundary for the single component DSMC simulation, above which heating can be ignored as the heating efficiency is assumed to go to zero. The DSMC results for escape $\phi$ and $\phi_{E}$ are then used in the next fluid solution. After a few 
exchanges between the fluid and DSMC models, a consistent solution is found for each specific heating case. I have solutions for no heating, solar minimum, and solar mean heating. Although non-thermal processes in the exobase region, $K n>0.1$, contribute to and can dominate the atmospheric loss rate (e.g. photo-disassociation, atmospheric sputtering), I ignore these here, consistent with previous work on escape from Pluto.

(ii) Since each DSMC simulation is quite time consuming, in the second hybrid model I find a fluid solution that is consistent with Jeans-like escape from the exobase., That is, in each iteration I find the exobase, $K n=1$, and update the escape rate and energy escape rate based on the Jeans equations (1.1-1.2) by using $\phi=K \times\langle\phi\rangle_{J}$ and $\phi_{E}=K \times\left\langle\phi_{E}\right\rangle_{J}$. Using $K=1$ results is normal Jeans escape, while $K>1$ is used to better reflect the results of molecular kinetic modeling that shows Jeans escape consistently underestimates the rate for the range of $\lambda$ of interest here (Merryfield and Shizgal, 1994; Volkov et al., 2011; Tucker et al., 2012). A consistent solution is obtained by stepping in time until convergence is obtained, which I define as when the 2-norm of the change in temperature, $\Delta T_{i}$, multiplied by the time step size divided by the number of radial grid points, $\frac{\Delta t}{N_{r}} \sqrt{\sum \Delta T_{i}^{2}}$, is less than $10^{-5}$.

The zero heating case was initiated using an isothermal profile and time stepped to steady state. Then I incrementally increased the solar flux rates to obtain the other 3 profiles in turn. As the exobase altitude increased with increase in the heating rate, the upper boundary altitude had to be increased to $16000 \mathrm{~km}$ (or about $14 r_{p}$ ) to model the solar maximum condition. 


\section{Thermosphere Model}

\subsection{Heating}

The radiative heating and cooling model is adapted from Strobel (2008), using the same fixed mixing ratios of $\chi_{N 2}=0.97, \chi_{C H 4}=0.03$, and $\chi_{C O}=0.00046$ for computing the heating and cooling rates. The energy fluxes and effective cross section for FUV absorption by $\mathrm{CH}_{4}$ and EUV absorption by $\mathrm{N}_{2}$ are given in Krasnopolsky (1999) for the various levels of solar activity at Pluto's perihelion (i.e. 30 AU). I obtain globally averaged heating rates for the FUV/EUV by applying Lambert-Beer's Law for a planeparallel atmosphere separately for each species $s$. Assuming an incoming energy flux $F_{S}^{\infty}$, the energy flux at a given altitude is given by

$$
F_{S}(r)=\frac{1}{2} \mu F_{S}^{\infty} \exp \left(-\tau_{s}(r) / \mu\right)
$$

where $\tau_{s}(r)=\int_{r}^{\infty} \sigma_{s} n_{s}(r) d r$ is the vertical optical depth, $\sigma_{s}$ is the absorption cross section of species $s, n_{s}$ is the number density of species $s$, and $\mu=\cos 60^{\circ}=0.5$ is used to approximate spherically averaged heating (Apruzese, 1980; Strobel et al., 1996). Then the heating rate for each species is given by

$$
q_{s}(r)=\frac{d F_{s}}{d r}=\sigma_{s} n_{s}(r) F_{s}^{\infty} \exp \left(-\tau_{s}(r) / \mu\right)
$$

Further heating and cooling mechanisms are provided by methane near-IR absorption and CO rotational line emission. In Strobel et al. (1996) the methane near-IR heating was treated as an non-LTE process, and the CO rotational line emission was 
shown to be an LTE process for pressures greater than $10^{-5} \mathrm{~Pa}$ while for larger $r$ it can become an non-LTE process. Parameterizations of these processes are given in Strobel, (2008), fit to the detailed radiative-transfer model of Pluto's lower atmosphere of Strobel et al. (1996). The methane heating rate per molecule is given as $8 \times 10^{-22} \exp \left(-\frac{\left(\lambda_{0}-\lambda\right)}{1.6}\right)$ $\operatorname{erg~s}^{-1}$, where $\lambda=\frac{G M m}{r k_{b} T}$ and $\lambda_{0}=\lambda\left(r_{0}\right)$. The CO rotational cooling rate per molecule is given as $8 \times 10^{-20}\left(\frac{T}{105}\right)^{2.45} \mathrm{erg} \mathrm{s}^{-1}$.

These calculations assume a fixed efficiency for the various processes, even though I know that this assumption will break down when the gas becomes tenuous. To accommodate this, I assume the heating rate is zero (i.e. the heating efficiency drops to zero) above $K n=0.1$. Furthermore, with the heating per molecule constant, including heating up to the top boundary can cause numerical instabilities even though the heating rate per unit volume is small. In describing the heating, a cut-off is often used where the tangential line-of-sight optical depth is unity (Strobel, 2008), which in our case lies below the $K n=0.1$ level.

To aid in convergence to steady state, I estimate the heating $q$ at the advanced time step using a Taylor expansion in $\xi$ as $q\left(r, \xi^{i+1}\right)=q\left(r, \xi^{i}\right)+\frac{d q}{d \xi}\left(\xi^{i+1}-\xi^{i}\right)$. This can help dissipate numerical oscillations between time steps, speeding up convergence. In calculating the derivative for the FUV and EUV heating I assume $\frac{d n}{d t}=0$ and $\frac{d \tau}{d t}=\frac{\tau}{T}$ as the optical depth $\tau \approx \sigma n H$ with $H$ depending linearly on $T$. 


\subsection{Results}

\subsubsection{Fluid-DSMC}

With the time-dependent solver, which is an improvement on the steady state iterations used in Tucker et al. (2012), I am able to model higher solar heating rates up to and including those at solar mean conditions. In Figure (3-1) I present the two previously calculated solutions found in that paper for zero heating and solar minimum heating along with a new Fluid-DSMC hybrid result for solar mean heating. This allows us to bracket the expected heating rates for the $\mathrm{NH}$ encounter. The hybrid solution includes rotational/ translational energy exchange consistent with the assumed thermal conductivity (see Tucker et al., 2012). Vibrational energy exchange of N2 is not included since the characteristic temperature is not in excess of $3000 \mathrm{~K}$ (Bird, 1993).

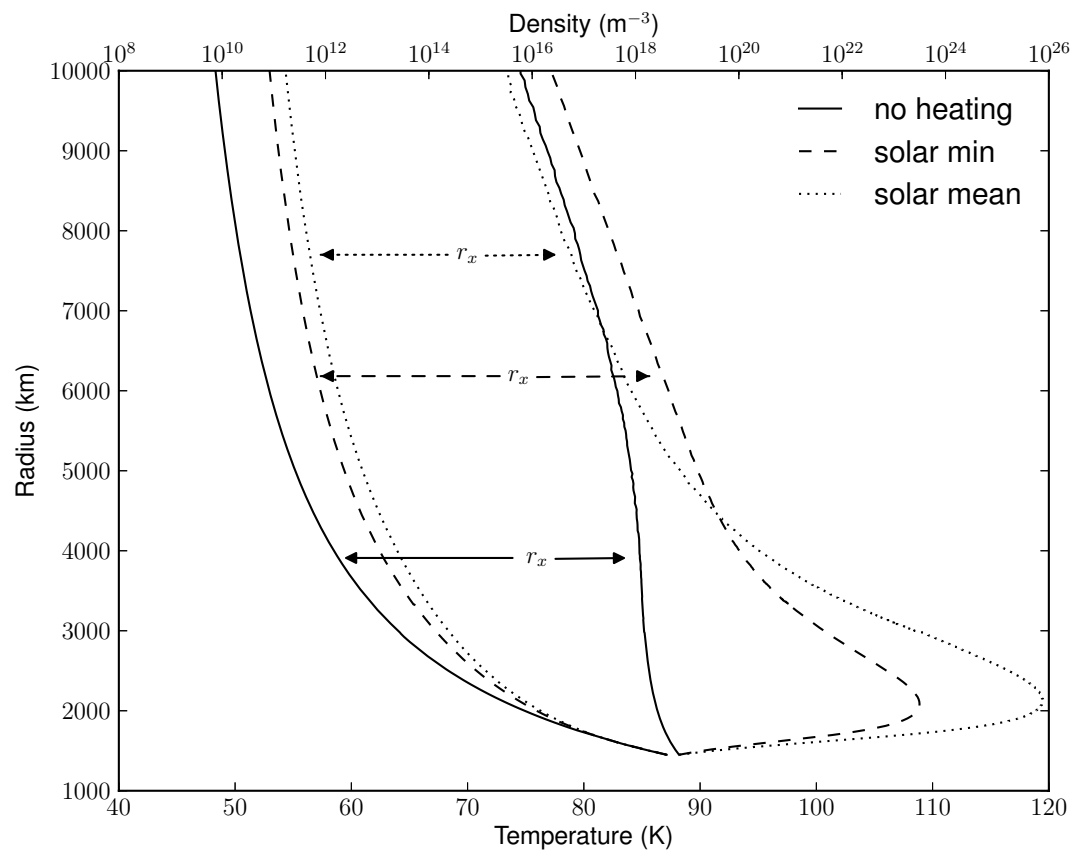

Figure 3-1: Temperature (right) and density (left) profiles for the hybrid Fluid-DSMC model of Pluto, for the cases of zero heating and solar minimum (from Tucker et al., 2012), and our new result for solar mean. The temperate profiles are plotted with the scale labeled below; the density profiles are plotted on a logarithmic 
scale, with the scale labeled above. The exobase altitudes are marked for each case by $\boldsymbol{r}_{x}$. The DSMC solutions are time-averaged after reaching steady state to smooth out random oscillations.

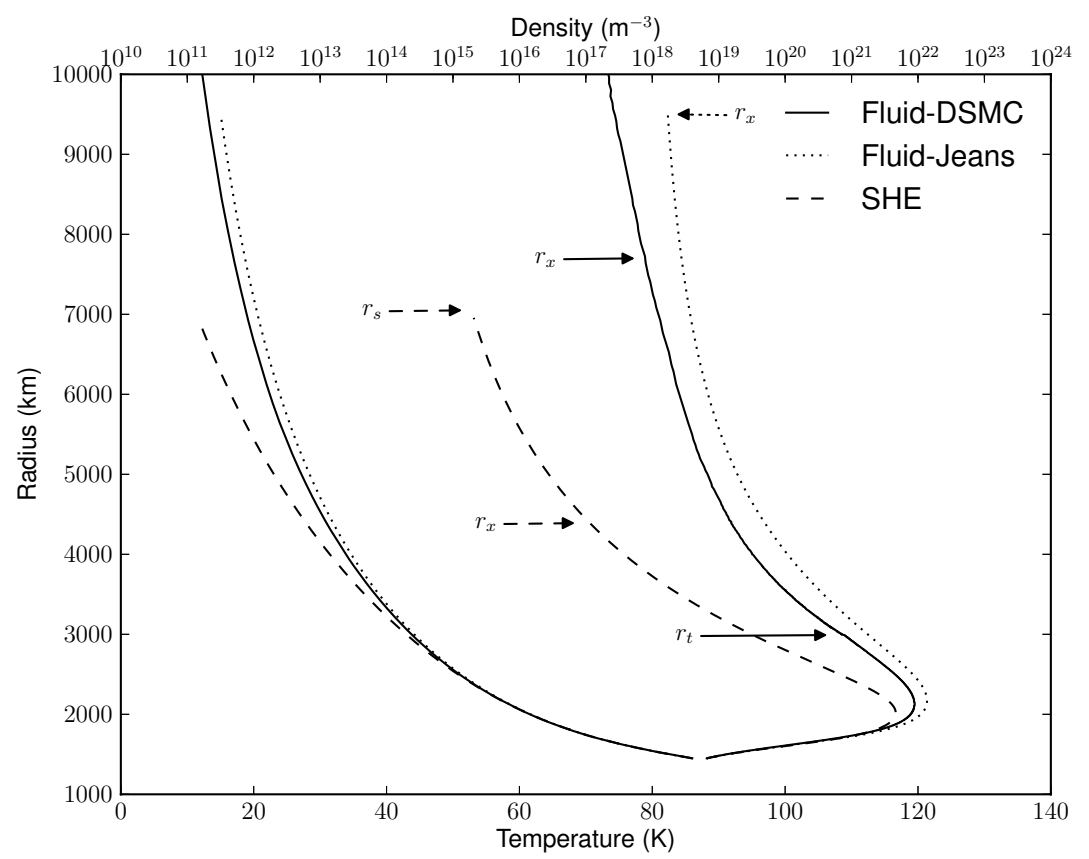

Figure 3-2: Temperature (right) and density (left) profiles for solar mean conditions, comparing the FluidDSMC solution of the present study with that of the SHE from Strobel (2008), along with the Fluid-Jeans. Marked on the Fluid-DSMC solution is the exobase $\left(r_{x}\right)$ as well as the transition between the fluid model and DSMC $\left(r_{t}\right.$, where $\left.K n\left(r_{t}\right)=0.1\right)$. Marked on the SHE model are the exobase $\left(r_{x}\right)$ and the sonic point $\left(r_{s}\right)$.

A few qualitative differences between the hydrodynamic solutions and our fluidDMSC solutions are seen in Figure (3-2). Although the two types of calculations give similar escape rates, they produce different temperature profiles as discussed in Volkov et al. (2011) and Tucker et al. (2012). The parallel and perpendicular temperature of the DSMC solutions differ above $K n \sim 0.1$, as seen in Tucker et al. (2012), again demonstrating failure in the region where continuum flow is expected to be inaccurate. More importantly for the $\mathrm{NH}$ encounter, the exobase altitude for the Fluid-DSMC solution is much higher (6000-10000 km depending on the heating rate) suggesting a significantly more extended atmosphere for Pluto than suggested by previous simulations. Although, the hydrodynamic methods can produce atmospheres that expand 
at supersonic speeds, I find the expansion is subsonic with the bulk flow velocity being less than $3 \%$ the speed of sound at the exobase (the speeds of sound are $\sim 180 \mathrm{~m} \mathrm{~s}^{-1}$ depending on temperature). Further out in the exosphere, the bulk speed increases to approach a constant value well below the speed of sound, consistent with a Jeans-like escape model.

In the solar mean case using the Fluid-DSMC model, the transition from the fluid to DSMC domain is near $3000 \mathrm{~km}$. In the region $0.1<K n<0.2$, the agreement between the fluid and DSMC solutions is better than $5 \%$ as in Tucker et al. (2012). The DSMC solution begins to deviate from the fluid solution above the $K n=0.2$ level. Here the kinetic and internal energies of the molecules begin to separate, which is associated with the transition to non-equilibrium flow. It is important to note that this occurs below the exobase, so the region of validity of the fluid equations should be terminated below the exobase. Our choice of $K n=0.1$ for connecting the DSMC solution to the fluid solution is at an altitude below which this separation occurs.

The solutions of Strobel (2008) result in exobase altitudes much lower than that found in our Fluid-DSMC model. His exobase altitudes are $\sim 0.56-0.7$ of those calculated with the hybrid model. With the lower exobase altitudes and the lower temperatures computed by Strobel (2008), the Jeans parameter at the exobase, $\lambda_{x}$, is $\sim 2.6-3.3$ times larger than found in this study. Using such a high $\lambda_{x}$ in Eq. (1.1) leads to a Jeans escape rate that is significantly lower than the total escape rate found; hence the earlier conclusion that escape rates were orders of magnitude larger than the Jeans escape rate. In contrast, using the exobase temperature calculate using the Fluid-DSMC model results in an escape rate that is only modestly enhanced relative to the calculated 
Jeans escape rate, the ratio $\phi /\langle\phi\rangle_{J}$ being 1.6, 2.0 and 2.3 for zero, solar minimum, and solar mean, respectively, as seen in Table 1.

\begin{tabular}{|c|l|l|l|}
\hline & Zero & Min. & Mean \\
\hline $\mathrm{Q}\left(10^{14} \mathrm{erg} \mathrm{s}^{-1}\right)$ & 0 & 3.8 & 7.8 \\
\hline$\phi\left(10^{27} \mathrm{~s}^{-1}\right)$ & 0.047 & 1.20 & 2.56 \\
\hline$E_{\text {esc }}\left(10^{-3} \mathrm{eV}\right)$ & 14.6 & 14.3 & 14.3 \\
\hline$r_{x} / r_{0}$ & 3.4 & 5.3 & 6.7 \\
\hline$\lambda_{x}$ & 8.9 & 5.7 & 4.8 \\
\hline$\phi /\langle\phi\rangle_{J}$ & 1.6 & 2.0 & 2.3 \\
\hline$\phi / \phi_{L}$ & - & 0.88 & 0.91 \\
\hline$E_{\text {esc }} /\left\langle E_{\text {esc }}\right\rangle_{J}$ & 0.95 & 0.90 & 0.97 \\
\hline
\end{tabular}

Table 1: Fluid-DSMC results. $E_{e s c}=\phi_{E} / \phi$ is the average energy carried off per molecule; $r_{x}$ is given as a ratio to $r_{p}=1150 \mathrm{~km} .\langle\phi\rangle_{J}$ is computed using exobase values from the solution, $\phi_{L}$ is computed using $Q$ from the solution and $r=1450 \mathrm{~km}$.

The results in Figure 3.1 and Table 1 correspond to an integrated net solar heating/cooling, $Q=\int_{r_{0}}^{r_{x}} 4 \pi r^{2} q(r) d r$, of $0.0,3.8$, and $7.8 \times 10^{14} \mathrm{erg} \mathrm{s}^{-1}$ for zero, solar minimum, and solar mean, respectively. These can be compared to $0.0,3.4$, and $6.9 \times 10^{14} \mathrm{erg} \mathrm{s}^{-1}$ for zero, solar minimum, and solar mean, respectively, found in Strobel (2008). To give context for these heating rates, the upward flow of energy across Pluto's $1450 \mathrm{~km}$ level would be greater than $10^{20} \mathrm{erg} \mathrm{s}^{-1}$ if it were escaping hydrodynamically (i.e. $\lambda \rightarrow 0)$. 
Many of the relevant values of the Fluid-DSMC hybrid solution in Tucker et al. (2012), as well as those for the new solar mean heating case, can be found in Table 1. It is seen that the escape fluxes are comparable to the Jeans flux and are also, quite remarkably, comparable to our estimate of the energy limited rate with the exception, of course, of the $Q=0$ case. This result alone is important because it is often presumed that the Jeans escape rate and the energy-limited rate are two limiting cases for atmospheric loss.

As in Tucker et al. (2012), for zero heating our escape rate is a order of magnitude lower than the SHE solution. For solar minimum our total escape rate is $20 \%$ below the SHE solution, and for solar mean I am slightly above. In each of these simulation cases, somewhat more heat is deposited than in Strobel (2008) due to our higher density and highly extended atmosphere. If the current simulation were scaled to the same heating rates the Fluid-DSMC escape rate would be slightly smaller than the SHE model as the DSMC simulations produce higher temperatures in the exosphere region, which imply lower adiabatic cooling and hence lower escape rate.

\subsubsection{Fluid-Jeans}

The Fluid-DSMC solutions demonstrate that the escape rate and energy escape rate are within a factor of two of the Jeans escape rate calculated from Eqs. (1.1) and (1.2). Therefore, in Figure 3.3 and Table 2 I present results for the Fluid-Jeans solution using an upper boundary condition that is equal to the Jeans escape and associated energy loss rates, and then doubling those. I note that average energy carried off per molecule in the 
Fluid-DSMC simulations is near that predicted by Jeans escape. By scaling both the molecular and energy escape rates by the same constant, the energy per molecule is not scaled. In Figure 3.2, it is seen that the Fluid-Jeans solution is in many respects similar to that of Fluid-DSMC solution (i.e. high temperature in upper thermosphere, extended exobase, etc.). Therefore, approximating the escape process as Jeans-like for Pluto's atmosphere under solar heating conditions likely occurring at the $\mathrm{NH}$ encounter reproduces many of the properties of the full solution in which I am interested. Although the fluid equations may not be valid up to the exobase, using them along with Jeans escape boundary conditions allows us to carry out parameter studies that are impractical due to computational cost with the full Fluid-DSMC model.

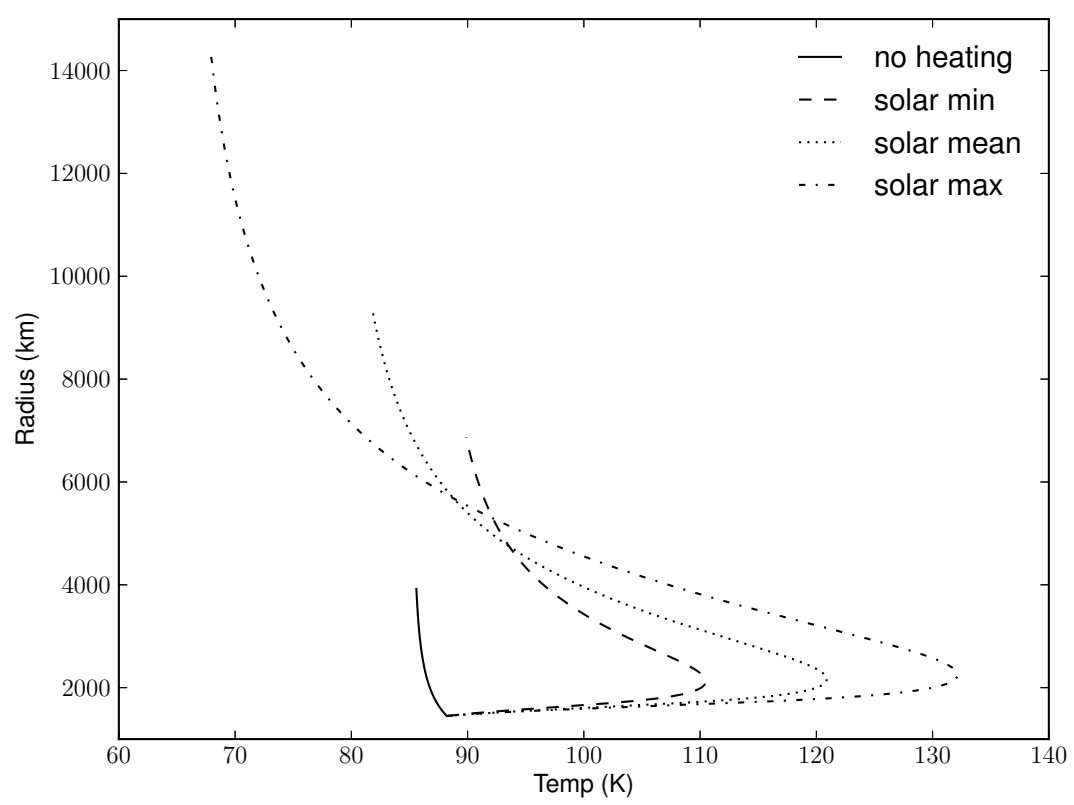

Figure 3-3: Temperature profile found in the Fluid-Jeans model for the 4 special cases discussed: zero heating, solar minimum, solar mean, and solar maximum. The top of each curve represents the exobase altitude.

The Fluid-Jeans model using the Jeans escape rate from the exobase results in a total escape rate that is consistent with the Fluid-DSMC model, but several properties of the Fluid-Jeans solution differ from the fluid-DMSC solution. For instance, the 
atmosphere is more extended than in the Fluid-DSMC model, causing slightly more EUV heat to be absorbed.. The Jeans parameter at the exobase is lower than for Fluid-DSMC model. These differences increase with escape rate.

\begin{tabular}{|c|c|c|c|}
\hline & \multicolumn{3}{|c|}{ Using $\phi=1 \times\langle\phi\rangle_{J}$} \\
\hline & Zero & Min. & Mean \\
\hline $\mathrm{Q}\left(10^{14} \mathrm{erg} \mathrm{s}^{-1}\right)$ & 0 & 3.9 & 8.9 \\
\hline$\phi\left(10^{27} \mathrm{~s}^{-1}\right)$ & 0.035 & 1.14 & 2.58 \\
\hline$E_{e s c}\left(10^{-3} \mathrm{eV}\right)$ & 15.5 & 16.3 & 14.6 \\
\hline$r_{x} / r_{0}$ & 3.41 & 5.99 & 8.10 \\
\hline \multirow[t]{3}{*}{$\lambda_{x}$} & 8.71 & 4.72 & 3.84 \\
\hline & \multicolumn{3}{|c|}{ Using $\phi=2 \times\langle\phi\rangle_{J}$} \\
\hline & Zero & Min. & Mean \\
\hline $\mathrm{Q}\left(10^{14} \mathrm{erg} \mathrm{s}^{-1}\right)$ & 0 & 3.8 & 7.8 \\
\hline$\phi\left(10^{27} \mathrm{~s}^{-1}\right)$ & 0.054 & 1.17 & 2.58 \\
\hline$E_{e s c}\left(10^{-3} \mathrm{eV}\right)$ & 15.2 & 15.4 & 13.7 \\
\hline$r_{x} / r_{0}$ & 3.3 & 5.3 & 6.8 \\
\hline$\lambda_{x}$ & 9.0 & 5.6 & 4.7 \\
\hline
\end{tabular}

Table 2: Results of the Fluid-Jeans model. The first results uses Jeans escape rate values for $\phi$ and $\phi_{E}$, while the second set uses twice the Jeans escape rate values. The exobase radius, $r_{x}$, is given a ratio relative to $r_{p}=1150$ km.

To more accurately model the Fluid-DSMC escape rate, I also present in Table 2 results obtained by scaling the Jeans escape values by a factor of 2 for the upper boundary, approximating the enhancement seen in the Fluid-DSMC solutions in Table 2. With this enhancement, the escape rate, exobase altitude, and exobase Jeans parameter 
more closely resemble the Fluid-DSMC results in Table 1. Surprisingly, ignoring the zero heating case, the converged escape rate is barely affected by the enhancement of the Jeans boundary conditions. Similarly, increasing the enhancement to 3 and 4 times the Jeans escape values increases the escape rate only a few percent, while the exobase altitude and temperatures in the upper atmosphere decrease significantly. Since the escape rate is roughly given by the energy-limited approximation, this is consistent with the loss rates being insensitive to the size of the enhancement in the Jeans boundary conditions. Although the other atmospheric properties adjust to ensure a nearly constant escape rate I would not have had an estimate of the enhancement in the escape rate relative to Jeans to accurately predict the atmospheric structure in the absence of the Fluid-DSMC simulations. Our early DSMC simulations indicated that for the case of zero heating above the lower boundary of the simulation region, the enhancement in total escape rate is $\sim 1.6$ (Volkov et al., 2011). How the escape rate enhancement increases with heating remains an open problem.

As the heating rate increases, the atmosphere gets further extended and $\lambda$ decreases to $\sim 3$ at the exobase for the solar maximum case using no enhancement to the total escape rate relative to Jeans escape rate. This is within the region that Volkov et al. (2011) found marks the transition to hydrodynamic escape for $\mathrm{N}_{2}$ at the relevant temperatures. In that study, it was shown that for the lower boundary at small $K n$, the exobase moves out to infinity as the Jeans parameter approaches $\sim \frac{c_{p}}{k_{b}}=3.5$. Using twice the Jeans escape values from Eqs. (1.1) and (1.2) as the boundary conditions causes the Jeans parameter in the upper atmosphere to increase, keeping the solution from entering the transonic regime. 


\begin{tabular}{|c|l|l|l|}
\hline \multicolumn{1}{|c|}{$\phi \mid\langle\phi\rangle_{J}$} & 1.0 & 2.0 & 3.0 \\
\hline $\mathrm{Q}\left(10^{14} \mathrm{erg} \mathrm{s}^{-1}\right)$ & 15.8 & 15.7 & 15.6 \\
\hline$\phi\left(10^{27} \mathrm{~s}^{-1}\right)$ & 5.81 & 5.80 & 5.79 \\
\hline$E_{\text {esc }}\left(10^{-3} \mathrm{eV}\right)$ & 11.7 & 10.9 & 10.4 \\
\hline$r_{x} / r_{0}$ & 12.4 & 9.8 & 8.9 \\
\hline$\lambda_{x}$ & 3.0 & 4.0 & 4.5 \\
\hline
\end{tabular}

Table 3: Fluid-Jeans model results for solar maximum case. Results are given for $1 \times, 2 \times$, and $3 \times$ the Jeans escape values as an upper boundary condition. The last column $(3 \times$ Jeans $)$ is an estimate of the enhancement, inferred from the Fluid-DSMC results in Table 1.

Based on the escape rate enhancement as a function of heating rate found in Table

3 , the escape rate for solar maximum condition is estimated to be close to 3 times the Jeans escape rate. If I accept the assumption that the enhancement will be 3.0 times Jeans for the solar maximum case then I can use the Fluid-Jeans model to discuss the atmosphere. In Table 3 the heating rate has approximately doubled relative to solar mean and the escape rate has likewise approximately doubled. The average energy carried off per molecule has decreased to $\sim 11 \times 10^{-3} \mathrm{eV}$, a decrease $\sim 30 \%$ relative to solar mean. The Fluid-Jeans solutions consistently underestimate the energy carried off per molecule, so the values given are likely lower than the values a Fluid-DSMC solution would give. The energy-limited escape rate $\phi_{L}=5.61 \times 10^{27}$ for this $Q$ is close to our estimated escape rate.

In Figure 3, the results for the four cases considered here using the Fluid-Jeans model are shown. I see that unlike the exobase altitude (that varies from $\sim 4000-$ $14000 \mathrm{~km})$, the altitude of the peak translational temperature does not increase significantly with heating rate, changing from $2124 \mathrm{~km}$ to $2250 \mathrm{~km}$ from solar minimum 
to solar max with translational temperatures of $110 \mathrm{~K}$ and $132 \mathrm{~K}$, respectively. The altitude of the heating peak is below that of the temperature maximum, and also does not change significantly with heating rate, changing from $1767 \mathrm{~km}$ to $1780 \mathrm{~km}$ from solar minimum to solar maximum, respectively. The altitude of the heating peak is near where the $\mathrm{CH}_{4}$ optical depth in the FUV is unity. The Fluid-Jeans solutions become nearly isothermal at the exobase consistent with the Fluid-DSMC model, but differ from the Parker (1964) and SHE models (McNutt, 1989; Watson et al., 1981; Krasnopolsky, 1999; Strobel, 2008) that require the temperature to decrease to zero at infinity.

Tian et al. (2008) found two regimes of escape in response to increased UV heating in his fluid dynamic studies of an early Earth atmosphere using the Jeans escape rate for the upper boundary condition. The transition from hydrostatic equilibrium to what he calls hydrodynamic escape coincides with adiabatic cooling becoming nonnegligible when compared to conduction or radiative heating. In our simulations of Pluto's atmosphere, adiabatic cooling is always an important cooling mechanism in the upper atmosphere. Furthermore, the changes in exobase altitude, temperature and bulk velocity with increased heating are similar to hydrodynamic escape regime of Tian et al. (2008), even though our escape is described as a kinetic process. Adiabatic cooling is always important in our simulations likely due to the much smaller gravitational binding energy of Pluto compared to Earth. The similarities with Tian et al. (2008) mentioned are likely due to his use of the Jeans escape rate at the upper boundary, which I have shown can be a reasonable approximation to the kinetic boundary conditions. 

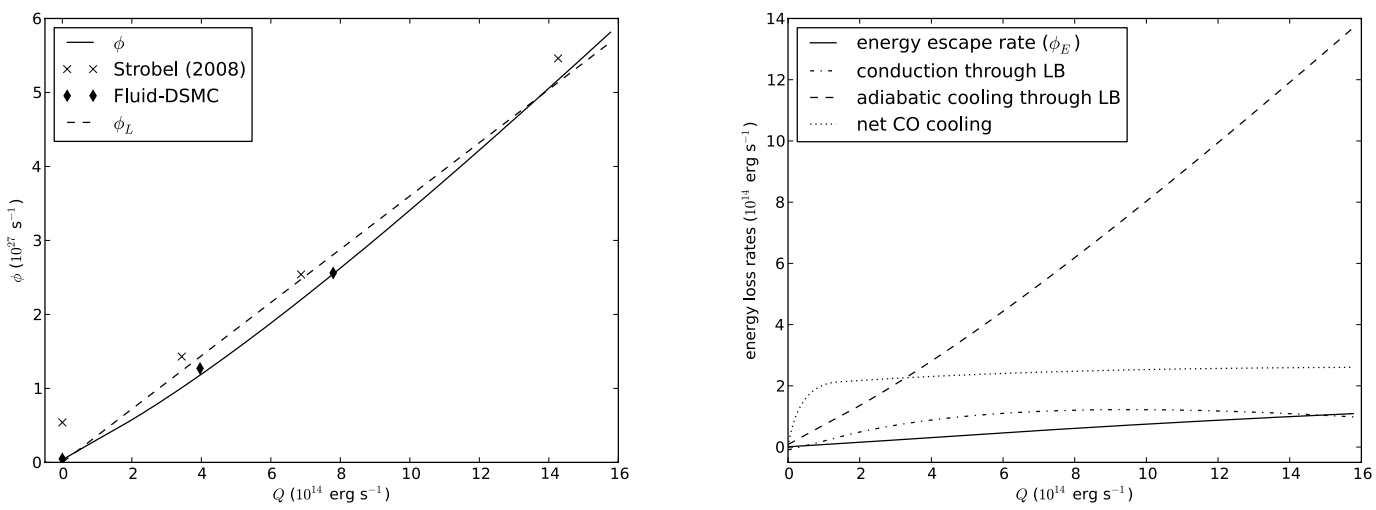

Table 4: (Left) Escape rate $\phi$ (solid line) plotted versus net heat deposited $Q$ from the Fluid-Jeans model. Overlaid are escape rates from the Fluid-DSMC model as black diamonds, and from the SHE model (Strobel, 2008) as $x^{\prime}$ s. Also plotted is the energy limited escape rate $\phi_{L}=Q / U\left(r_{0}\right)$ (dashed line). (Right) Comparison energy loss mechanisms versus net heat deposited from the Fluid-Jeans model.

In Figure 4, the escape rate versus the net heating rate for the Fluid-Jeans model is compared to the Fluid-DSMC and SHE models of Strobel (2008). The Fluid-DSMC results are nearly the same as the Fluid-Jeans results, thus confirming that the Fluid-Jeans model can be used to estimate the escape rate for this range of Jeans parameters. With the exception of the zero heating case, the SHE model results show a similar trend but are $43 \%, 16 \%$ and $7 \%$ higher than the Fluid-Jeans for solar minimum, mean, and maximum, respectively, with the latter approaching blow-off.

Also shown in Figure 4 is the energy-limited escape rate compared to the FluidJeans escape rate. The good agreement between the two escape models suggests that most of the energy being deposited is going into removing the molecules from the gravitational well. The sources of cooling besides adiabatic expansion and loss to escape are conduction through the lower boundary and CO cooling (already included in $Q$ ) both of which are bounded, as found in the hydrodynamic model (Strobel, 2008). The slope of the energy-limited escape rate can be better matched to our simulations by accounting for the internal energy (i.e. $C_{p}\left(T_{0}-T_{x}\right)$ ). The vertical offset can be partly compensated for 
by subtracting the loss to conduction through the lower boundary from the total energy deposited, but since it is bounded it is a small correction to the escape rate.

\begin{tabular}{|c|c|c|c|}
\hline & \multicolumn{3}{|c|}{$F^{\infty}=1 \times 10^{-3} \mathrm{erg} \mathrm{cm}^{-2} \mathrm{~s}^{-1}$} \\
\hline & $\phi\left(10^{27} \mathrm{~s}^{-1}\right)$ & $r_{h} / r_{0}$ & $Q\left(10^{14} \mathrm{erg} \mathrm{s}^{-1}\right)$ \\
\hline Fluid-Jeans & 1.06 & 1.22 & 4.68 \\
\hline Watson & 15.4 & 3.66 & 35.4 \\
\hline McNutt & 0.84 & 1.17 & 3.59 \\
\hline ELE (a) & 1.69 & - & 4.68 \\
\hline ELE (b) & 1.66 & 1.32 & 4.62 \\
\hline $\operatorname{ELE}(\mathrm{c})$ & 1.41 & 1.22 & 3.94 \\
\hline \multirow[t]{3}{*}{ ELE (d) } & 0.95 & 1.0 & 2.64 \\
\hline & \multicolumn{3}{|c|}{$F^{\infty}=4 \times 10^{-3} \mathrm{erg} \mathrm{cm}^{-2} \mathrm{~s}^{-1}$} \\
\hline & $\phi\left(10^{27} \mathrm{~s}^{-1}\right)$ & $r_{h} / r_{0}$ & $Q\left(10^{14} \mathrm{erg} \mathrm{s}^{-1}\right)$ \\
\hline Fluid-Jeans & 8.46 & 1.25 & 23.3 \\
\hline Watson & 32.1 & 2.73 & 78.6 \\
\hline McNutt & 3.22 & 1.28 & 17.4 \\
\hline ELE (a) & 8.40 & - & 23.3 \\
\hline ELE (b) & 7.30 & 1.39 & 20.3 \\
\hline $\operatorname{ELE}(\mathrm{c})$ & 5.91 & 1.25 & 16.4 \\
\hline ELE (d) & 3.81 & 1.0 & 10.5 \\
\hline
\end{tabular}

Table 5: Comparing the Fluid-Jeans model, Hunten and Watson (1982), McNutt (1989) and energy-limited escape (ELE). For each model, the values for each given are the escape rate $\phi$, the location of the heating layer in terms of the lower boundary $r_{h} / r_{0}$ (in the case of the Fluid-Jeans model $I$ give the location of the peak heating, which is where the slant optical depth is unity), and the net heating $Q$ are shown. The ELE model was applied in 4 ways: (a) using $Q$ from the Fluid-Jeans model, (b) $r_{h}$ is altitude of unit parallel optical depth, (c) $r_{h}$ is the altitude of unit vertical optical depth, (d) and $r_{h}$ is $r_{0}$ as if no estimate were given. 
I also compared escape rates from the Fluid-Jeans model, the hydrodynamic models of Hunten and Watson (1982) and McNutt (1989), and the energy-limited escape model of Lammer et al. (2009) for the same solar flux. For a consistent comparison I adopt a single solar heating source for methane with a globally averaged incoming solar energy flux, $F^{\infty}$, of $1 \times 10^{-3}$ and $4 \times 10^{-3} \mathrm{erg} \mathrm{cm}^{-2} \mathrm{~s}^{-1}$ (representing near solar minimum and maximum conditions respectively), and an absorption cross-section for $\mathrm{CH} 4$ of $1.8 \times 10^{-17} \mathrm{~cm}^{2}$. I assume a primarily nitrogen atmosphere with the values for conductivity used in this paper, $\kappa_{0}=9.38 \times 10-5 \mathrm{~J} \mathrm{~m}^{-1} \mathrm{~K}^{-1}$, and a fixed $\mathrm{CH}_{4}$ mixing ratio of $3 \%$ when calculating the optical depth.

In Table 5, neither of the simple hydrodynamic models produces a similar escape rate to the Fluid-Jeans model for either heating case. The Hunten and Watson (1982) model is an upper bound on the escape rate, so it will consistently overestimate the escape rate. The McNutt (1989) is a good estimate for the smaller heating rate, giving a similar estimate of both the escape rate and the altitude of the heating layer; however, it is quite different from the Fluid-Jeans for the solar maximum case. While the hydrodynamic models can give good estimates of the escape rate for certain cases, the atmospheric structure of the solutions are quite different from that found the Fluid-Jeans or Fluid-DSMC models.

The energy-limited escape model can be a reasonable fit if the net heat deposited in the atmosphere is known. Though without detailed modeling, one can only estimate the amount of energy deposited. In Table 5 I consider calculating the energy-limited escape rate using 4 different estimates of the net amount of heat deposited: i) the net heating rate is the net heating deposited in Fluid-Jeans solution, ii) the net heating rate is 
$Q=\pi r_{h}^{2} F^{\infty}$ with the radius of the heating layer $\left(r_{h}\right)$ being the radius of unit parallel optical depth found using the Fluid-Jeans model of the atmosphere, iii) with the radius of the heating layer being the radius of unit vertical optical depth found using the FluidJeans model (this is also the altitude of the peak UV heating), iv) with the heat deposited at the lower boundary, $r_{h}=r_{0}$. When $Q$ is obtained from the Fluid-Jeans model, the energy-limited escape provides a good estimate for the higher heating rate; but for the near solar minimum rate it overestimates the escape rate as a significant amount of heat is conducted through the lower boundary. Using method (ii) above, it is seen that the parallel optical depth provides a good estimate of the heat deposited in both cases, but similar to (i) it does not provide a good estimate of the escape rate for the solar minimum case as a significant amount of this heat is again conducted through the lower boundary instead of driving escape. Methods (iii) and (iv) for estimate the energy limited rate fail to predict $Q$ as accurately as using the radius of unit parallel optical depth.

I also investigated the sensitivity of the Fluid-Jeans model to changes in some of the model parameters. If the atmosphere were a monatomic gas $\left(C_{p}=\frac{5}{2} k_{b}\right)$ rather than diatomic $\left(C_{p}=\frac{7}{2} k_{b}\right)$, then $E_{e s c}$ is about halved, primarily due to no internal energy being carried off by the escaping gas; however, the other properties are only significantly affected when the heating rate exceeds solar mean conditions. For solar mean, the escape rate increases by $\sim 1 \%$ and the exobase is lower by $\sim 10 \mathrm{~km}$. In hydrodynamic models it is often assumed that the energy carried off by escape is negligible and a positive non-zero energy loss can only decrease the escape rate (Watson et al, 1981). Using $\phi_{E}=0$ (keeping $\phi$ as the Jeans value), then the escape rate increases by $\sim 5-10 \%$ depending on solar flux. When the heating rate is at solar maximum conditions it is found that $\phi_{E}$ 
begins to become comparable to conduction or $\mathrm{CO}$ cooling, the exobase altitude is lowered from 14200 to $12600 \mathrm{~km}$, and the exobase temperature increases from 68 to $81 \mathrm{~K}$. Therefore, even though neglecting $\phi_{E}$ still produces a reasonable estimate of escape rate, it should be included when interested in the vertical structure of the atmosphere.

Another consideration is my definition of the Knudsen number used to determine the exobase altitude. The relevant length scale in the atmosphere is determined by the density gradient $H_{n}=-n /(d n / d r)$. The isothermal approximation $H=k_{-} b T / m g(r)$ was used in the above simulations. Using $K n=\ell / H_{n}$ to determine the exobase altitude results in a small correction (since $H \sim H_{n}$ throughout the atmosphere) and has a negligible effect on the solution and escape rate. Since the Fluid-Jeans atmosphere is highly extended one might consider $K n=\ell / r$, which is appropriate for comets (Cercignani, 2000), where the density drops off rapidly as $r^{-2}$. Using this definition, the exobase altitude increases to $4.7,9.3,13.3$, and $21.6 \times r_{p}$ for the four heating cases considered above. These values are about 50\% larger than those in Tables 2 and 3 and do not compare well with the Fluid-DSMC results. For solar maximum such an estimate would place the exobase beyond the orbit of Charon. 


\section{Full Atmosphere Model}

In this chapter I develop in collaboration with D. Strobel and X. Zhu a onedimensional, spherically symmetric model of Pluto's atmosphere from the surface up to and including the exosphere. This is another application of the hybrid fluid-kinetic models, including heating models relevant to both the lower and upper atmosphere. In this way no assumptions are made in the middle of the atmosphere, as is necessary when modeling the lower or upper atmosphere alone.

One-dimensional radiative-conduction models and global circulation models (GCM's), as well as simpler models, have been used to describe Pluto's lower atmosphere. Occultation studies like that of Elliot et al. (1989) use a simple temperature model to fit the measured light curve. In this case an isothermal model can be fit reasonably well between the top of the extinction layer $\sim 50 \mathrm{~km}$ above the surface and the top of the light curve that corresponds to $\sim 1500 \mathrm{~km}$ in radius. Yelle et al. (1989) used a radiative-conductive model, including the $3.3 \mu \mathrm{m}$ methane heating band and $7.8 \mu \mathrm{m}$ methane cooling band, and found that with a surface temperature of $\sim 50 \mathrm{~K}$ that the heating would produce a fast temperature increase to $\sim 106 \mathrm{~K}$ at the $1 \mu \mathrm{bar}$ level. This would be $\sim 2$ scale heights above the surface, or about $120 \mathrm{~km}$ in altitude, with a surface pressure of $10 \mu$ bar. At the upper boundary $\left(\sim 10^{-2} \mu\right.$ bar $)$ Yelle et al. (1989) set the heat flux equal to zero as an approximation, and found the upper portion of his solution to be nearly isothermal. Yelle et al. (1989) also noted that a large amount of the solar UV would be deposited at or above this level so that solving the hydrodynamics equations in the region as well would be necessary to better approximate the atmosphere. 
Strobel et al. (1996) used a radiative-conductive model and included more heating and cooling mechanisms than Yelle et al. (1989). They included the $2.3 \mu \mathrm{m}$ and $3.3 \mu \mathrm{m}$ methane-heating bands, the $7.6 \mu \mathrm{m}$ methane-cooling band (treating it non-LTE as well), and CO rotational cooling. Strobel et al. (1996) also used a zero flux upper boundary condition at $700 \mathrm{~km}$ in altitude and ignored UV heating. Zalucha et al. (2011) used the radiative-conductive model of Strobel et al. (1996) to fit measured light curves from stellar occultation. While this was very useful in finding a set of best-fit lower boundary pressure and mixing ratios, it still used the zero flux upper boundary condition and did not include escape or adiabatic cooling.

Models of the upper atmosphere that include escape have typically used a lower boundary in the atmosphere somewhere above the surface (Hunten et al., 1982; McNutt, 1989; Krasnopolsky, 1999; Strobel, 2008; Tucker et al., 2012, Erwin et al., 2013). In early models (Hunten et al., 1982; McNutt, 1989; Krasnopolsky, 1999) the stratospheric processes of methane IR heating and CO rotational cooling were ignored, and only the UV heating was considered. Strobel (2008) included a parameterization of these stratospheric processes based on the results of Strobel et al. (1996) in which these processes were solved in detail for the lower atmosphere. The same parameterizations were included in Tucker et al. (2012) and Erwin et al. (2013) to compare with previous modeling. The choice of lower boundary location and the local atmospheric values differ between these models, but most start at a radius between $1400 \mathrm{~km}$ and $1500 \mathrm{~km}$ where the Jeans parameter is between 20 and 30 . The energy conducted through the lower boundary varies with the amount of solar UV heating, and it is assumed that the atmosphere below the lower boundary is relatively unaffected by the energy conducted downward or by the 
energy lost to escape. Although these are common approximations when modeling planetary atmospheres, a full atmospheric model is needed to obtain a consistent solution between the lower and upper atmospheres. Such a model is constructed below.

\subsection{Heating models}

I include the stratospheric radiative heating and cooling models of Strobel et al. (1996), with the updates to the values given in Zalucha et al. (2011), that dominate the heating in the lower atmosphere. For the UV heating in the upper atmosphere, I use tabulated values for the cross section of nitrogen and methane and the measured solarspectral irradiance between 0 and $140 \mathrm{~nm}$ in place of the values adapted from Krasnopolsky (1999). The sign conventions given in the following sections are used to calculate the net radiative heating $q(r)$ in the form

$$
q(r)=q_{2.3}+q_{3.3}-q_{7.6}-q_{C O}+q_{U V}
$$

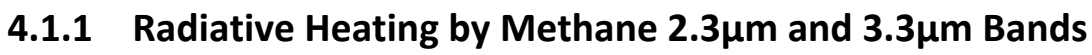

Each of the methane heating bands is comprised of multiple lines due to multiple vibrational bending and stretching transitions and de-excitations. In this section I present the equation used for each of the heating bands, summarizing the derivation and discussion given in Strobel et al. (1996).

The heating rate in the near-IR for a solar-zenith angle $\theta=\cos ^{-1} \mu$ is given by 


$$
q(\mu)=\frac{1}{2} S_{0} \epsilon n_{a} \int_{\Delta v} \sigma_{v} \exp \left(-\sigma_{v} N_{a} / \mu\right) d v
$$

Here $S_{0}$ is the solar energy flux and the $1 / 2$ accounts for the diurnal averaging, $\epsilon$ is the heating efficiency, $\sigma_{v}$ is the cross section at frequency $v, n_{a}$ and $N_{a}$ are the number density and column density of the absorbing molecule (i.e. $\mathrm{CH}_{4}$ ), and the integration is over the bandwidth $\Delta v\left(=400 \mathrm{~cm}^{-1}=3200-2800 \mathrm{~cm}^{-1}\right.$ for the $3.3 \mu \mathrm{m}$ band $)$. I introduce the escape function for photons in a given band, $\Delta v$ :

$$
\Gamma_{\mu}\left(N_{a}\right)=\frac{1}{\bar{\sigma} \Delta v} \int_{\Delta v} \sigma_{\nu} \exp \left(-\sigma_{v} N_{a} / \mu\right) d v
$$

Here $\bar{\sigma} \Delta v=\int_{\Delta v} \sigma_{v} d v$ is the band strength. Since I am computing the spherically average energy flux and the globally averaged temperature, I use a hemispherically averaged band escape function

$$
\Gamma_{\pi}=\frac{2}{\pi} \int_{0}^{\pi / 2} d \lambda \int_{0}^{\pi / 2} \Gamma_{\mu}\left(N_{a}\right) \cos \varphi d \varphi
$$

Here the solar zenith angle $\theta$ is related to the angles $\lambda$ and $\varphi$ by $\mu=\cos \theta=\cos \lambda \cos \varphi$. Note in Eq. (4.4) the integration that is normally over the full sphere ( $4 \pi \mathrm{sr})$, is only needed over the upward hemisphere $(2 \pi \mathrm{sr})$, and is further reduced to a quarter hemisphere $(\pi / 2 \mathrm{sr})$ using the symmetry of the integral. In practice the integration in Eq. (4.4) is performed by 4 point Gaussian integration on each angle, given 16 points total. Using these definitions the heating rate is expressed as 


$$
q=\frac{1}{2} S_{0} \epsilon n_{a}(\bar{\sigma} \Delta v) \Gamma_{\pi}\left(N_{a}\right)
$$

Computation of the escape function requires integration over a spectrum within each band. This could be done by line-by-line integration, but for methane bands this requires the integration over $>10^{5}$ lines per band; considering line broadening this becomes a computationally intensive problem. An efficient way of performing this integration is by the correlated k-distribution method. This method involves changing the variable of integration from frequency to a normalized wavenumber $\mathrm{g}$.

$$
\begin{gathered}
\int_{\Delta v} \sigma_{v} \exp \left(-\sigma_{v} N_{a}\right) d v=\Delta v \int_{0}^{\infty} \exp \left(-\sigma N_{a}\right) f(\sigma) d \sigma \\
=\Delta v \int_{0}^{1} \exp \left(-k_{g} N_{a}\right) d g
\end{gathered}
$$

Here $f(\sigma)$ is the inverse Laplace transform of the spectral transmittance, and $\mathrm{g}$ is the cumulative probability function of $f(\sigma)$ (Liou, 2002). One can think of $k_{g}$ as a cross section mapped to the normalize wavenumber $\mathrm{g}$ ranging from 0 to 1 . Further the kcoefficients change with temperature and pressure since these changes affect the line shape. In practice, a 30-point Gaussian quadrature computes the correlated k-coefficient integration

$$
\Gamma_{\mu}\left(N_{a}\right)=\frac{\sum_{j=1}^{30} w_{j} k_{j} \exp \left(-k_{j} N_{a} / \mu\right)}{\sum_{j=1}^{30} w_{j} k_{j}}
$$

The coefficients, $k_{j}$, and weights, $w_{j}$, are computed on 5 point Gaussian quadrature's on the subinterval of $1-\mathrm{g}$ with boundaries of $\left[0.0,10^{-5 / 2}, 10^{-2}, 10^{-3 / 2}, 10^{-1}, 10^{-1 / 2}, 1\right]$ as the 
shape of $k_{g}$ is highest in magnitude and fastest changing at the higher values of $g$ (Zhu 1992). Strobel et al. (1996) computed the k-coefficients for the $2.3 \mu \mathrm{m}$ and $3.3 \mu \mathrm{m}$ bands for temperatures $40,60,80$, and $100 \mathrm{~K}$. The $k_{g}$ used in equation (4.7) are exponentially interpolated from these values to the required temperature. As pressures are small on Pluto, pressure broadening is negligible and was not considered.

I introduce the notational $\Gamma_{\pi, i}\left(N_{a}\right)$ for the hemispherically averaged escape function with the additional subscript denoting the absorption band. The convention will be $i=2$ for the $2.3 \mu \mathrm{m}$ band, $i=3$ for the $3.3 \mu \mathrm{m}$ band, and $i=4$ for the $7.6 \mu \mathrm{m}$ band that will be discussed in the next section. Also necessary for the calculation for the heating rates is the photo-flux escape function for a plane-parallel atmosphere $\Gamma_{f, i}\left(N_{a}\right)$. It is computed for each band as follows:

$$
\begin{gathered}
\Gamma_{f}\left(N_{a}\right)=\int_{0}^{1} \Gamma_{\mu}\left(N_{a}\right) d \mu=\frac{1}{\bar{\sigma} \Delta v} \int_{\Delta v} \sigma_{v} E_{2}\left(\sigma_{v} N_{a}\right) d v \\
\Gamma_{f}\left(N_{a}\right)=\frac{\sum_{j=1}^{30} w_{j} k_{j} \mathrm{E}_{2}\left(k_{j} N_{a}\right)}{\sum_{j=1}^{30} w_{j} k_{j}}
\end{gathered}
$$

Here $E_{2}(x)$ is the exponential integral, a mathematic function available in most modern codes (e.g. Fortran), and a discussion of which can be found in Abromowitz and Stegun (1965). In an optically thick atmosphere, the flux escape function alone can be used to approximate the heating efficiency.

Now the heating rate for the 3.3 micron band can be expressed from Eqn. (4.5) as

$$
q_{3.3}=\frac{1}{2} S_{0} \epsilon_{3.3} n_{a}(\bar{\sigma} \Delta v) \Gamma_{\pi, 3}\left(N_{a}\right)
$$


The line solar energy flux for the band at $30 \mathrm{AU}$ is $S_{0}=0.022 \mathrm{erg} \mathrm{cm}^{-1} \mathrm{~s}^{-1}$, and the band strength is $\bar{\sigma} \Delta v=1.17 \times 10^{-17} \mathrm{~cm}$.

The efficiency factor $\epsilon$ for conversion of absorbed photon energy for each of the bands to molecular translational energy can be calculated by considering relaxation pathways between the various vibrational states. For instance, the $3.3 \mu \mathrm{m}$ absorption is due to vibrational excitation states $\left(v_{1}, 2 v_{2}, v_{3}, v_{2}+v_{4}\right)$, but since exchange between these states is extremely fast, Strobel et al. (1996) treated them as a single level with the properties of $v_{3}$ excitation, which has the strongest absorption.

The non-LTE efficiency factor for the band sequence $\left(v_{3} \rightarrow 2 v_{4} \rightarrow 2 \times\right.$ $v_{4} \rightarrow$ ground level) is then approximated as

$$
\epsilon_{3.3}=\frac{1}{1+\Gamma_{f_{3}}\left(N_{a}\right) \phi_{3}^{-1}}\left[\beta_{3.3}+\frac{1}{1+\phi_{2 v_{4}}^{-1}} \frac{\left(1-\beta_{3.3}\right)}{1+\Gamma_{f_{4}}\left(N_{a}\right) \phi_{4}^{-1}}\right]
$$

The first term $\beta_{3.3}=\frac{v_{3}-2 v_{4}}{v_{3}}=0.13$ represents the partial thermalization of the initial photon energy through a $(\mathrm{V}-\mathrm{V})$ transition from the stretching to bending vibrational modes. The second term is the thermalization of the other $87 \%$ of the photon energy through a (V-T) transition from the vibrational to rotational/translational modes. The $\phi$ 's represent the ratios of collisional deactivation $\left(k_{i} n\right)$ to spontaneous radiative decay $\left(A_{i}\right)$ of the different vibrational states, and are given by 


$$
\begin{gathered}
\phi_{3}=\frac{k_{3} n}{A_{3}}=\frac{2.8 \times 10^{-11} n}{25.2} \\
\phi_{2 v_{4}}=\frac{k_{3} n}{2 A_{4}}=\frac{1.16 \times 10^{-11} n}{2 \times 2.12} \\
\phi_{4}=\frac{k_{4} n}{A_{4}}=\frac{1.9 \times 10^{-15}\left(1+10 \chi_{C H 4}\right) \exp \left(\frac{T-240}{70}\right) n}{2.12}
\end{gathered}
$$

In Eqns. (4.12), the de-excitation rates, $k_{i}$, are given in $\mathrm{cm}^{3} \mathrm{~s}^{-1}$, the Einstein coefficients, $A_{i}$, are given in $\mathrm{s}^{-1}$, and the density $n$ is the total local density given in $\mathrm{cm}^{-3}, \mathrm{~T}$ is the local temperature in Kelvin, and $\chi_{\mathrm{CH} 4}$ is the methane-mixing ratio.

Similarly, using Eq. (4.5) the heating rate for the $2.3 \mu \mathrm{m}$ heating band (4000$4600 \mathrm{~cm}^{-1}$ ) can be expressed as

$$
q_{2.3}=\frac{1}{2} S_{0} \epsilon_{2.3} n_{a}(\bar{\sigma} \Delta v) \Gamma_{\pi, 2}\left(N_{a}\right)
$$

The solar energy flux for this band at $30 \mathrm{AU}$ is $S_{0}=0.037 \mathrm{erg} \mathrm{cm}^{-1} \mathrm{~s}^{-1}$, and the band strength is $\bar{\sigma} \Delta v=2.03 \times 10^{-18} \mathrm{~cm}$. The non-LTE efficiency for the $2.3 \mu \mathrm{m}$ factor for the band sequence $\left(v_{3}+v_{4} \rightarrow 3 v_{4} \rightarrow 3 \times v_{4} \rightarrow\right.$ ground level $)$ is used

$$
\epsilon_{2.3}=\frac{1}{1+\Gamma_{f_{2}}\left(N_{a}\right) \phi_{v 3}^{-1}}\left[\beta_{2.3}+\frac{1}{1+\phi_{3 v_{4}}^{-1}} \frac{\left(1-\beta_{2.3}\right)}{1+\Gamma_{f_{4}}\left(N_{a}\right) \phi_{4}^{-1}}\left(1+\frac{2}{1+2 \phi_{2 v_{4}}^{-1}}\right)\right]
$$

The fraction of $(\mathrm{V}-\mathrm{V})$ thermalization for this band is $\beta_{2.3}=\frac{v_{2.3}-3 v_{4}}{v_{2.3}}=0.09$. Further deactivation ratios are 


$$
\begin{aligned}
& \phi_{v 3}=\frac{k_{3} n}{A_{2}}=\frac{2.8 \times 10^{-11} n}{27.3} \\
& \phi_{3 v 4}=\frac{k_{3} n}{3 A_{4}}=\frac{2.8 \times 10^{-11} n}{3 \times 2.12}
\end{aligned}
$$

As in Eqn. (4.15), the density is assumed to be in units of $\mathrm{cm}^{-3}$.

The expression for the heating efficiencies, Eqns. (4.11) and (4.14), and the values for the deactivation rates in Eqns. (4.12) and (4.15) are from Zalucha et al. (2011) using updated expressions given in Boursier et al. (2003). The expression used for $k_{4}$ was given in Siddles et al. (1994) to better approximate the collisional deactivation of the $v_{4}$ vibrational band of methane at low temperatures.

\subsubsection{Non-LTE Radiative Cooling by Methane 7.6 $\mu \mathrm{m}$ Band}

The non-LTE radiative cooling by methane $7.6 \mu \mathrm{m}$ is computed as in Strobel et al. (1996) by the correlated k-distribution method using the Curtis matrix formulation given in Zhu (1990). The source of the cooling is blackbody emission of the methane $v_{4}$ band being transmitted between levels and lost to the surface or space. Since the Planck black body function varies significantly over the band $\left(1990-1360 \mathrm{~cm}^{-1}\right)$ the band is divided into the subbands $\left(1090-1225 \mathrm{~cm}^{-1}\right)$ and $\left(1225-1360 \mathrm{~cm}^{-1}\right)$. The line centers, $\bar{v}$, are $1157.5 \mathrm{~cm}^{-1}$ and $1292.5 \mathrm{~cm}^{-1}$, respectfully, and the band strengths, $\sigma \Delta v$, are $1.62 \times 10^{-21} \mathrm{~cm}$ and $5.20 \times 10^{-18} \mathrm{~cm}$, respectfully, for the two subbands. Correlated k-coefficients were derived for these two bands separately, again for temperatures $40,60,80$, and $100 \mathrm{~K}$ (Strobel et al., 1996). 
As the transmission between levels is of upmost importance the flux escape function for the $v_{4}$ band between the atmospheric levels is

$$
\begin{gathered}
\Gamma_{f, 4}\left(z, z^{\prime}\right)=\frac{\int_{0}^{1} k_{g}(r) \mathrm{E}_{2}\left(\Delta u_{g}\left(r, r^{\prime}\right)\right) d g}{\int_{0}^{1} k_{g}(r) d g} \\
\Gamma_{f, 4}\left(z, z^{\prime}\right)=\frac{\sum_{j=1}^{30} w_{j} k_{j} \mathrm{E}_{2}\left(\Delta u_{g_{j}}\left(r, r^{\prime}\right)\right)}{\sum_{j=1}^{30} w_{j} k_{j}}
\end{gathered}
$$

The integrals in Eq. (4.16) are computed by the correlated k summation in Eq. (4.17) using the same quadrature points and weights as those used in flux escape functions for the $2.3 \mu \mathrm{m}$ and $3.3 \mu \mathrm{m}$ (Eqns. (4.7) and (4.9)), but separately for each subband. The optical thickness $\Delta u_{g}$ is expressed by

$$
\Delta u_{g}\left(r, r^{\prime}\right)=\left|\int_{r}^{r \prime} k_{g}\left(r^{\prime \prime}\right) n_{a}\left(r^{\prime \prime}\right) d r^{\prime \prime}\right|
$$

The flux escape function $\Gamma_{f, 4}$ used in computing the non-LTE efficiencies for the $2.3 \mu \mathrm{m}$ and $3.3 \mu \mathrm{m}$ bands is the escape function to infinity for the entire $v_{4}$ band. It is computed by taking the weighted average of $\Gamma_{f, 4}\left(r, r_{t}\right)$, where, of each subband, with the weights being the band strength for the respective subband. While this is the band escape function from level $r$ to the upper boundary $r_{t}$, since the column density above $r_{t}$ is extremely small compared to the column density near the surface where the $2.3 \mu \mathrm{m}$ and $3.3 \mu \mathrm{m}$ bands are most significant, the difference is negligible.

The cooling rate is then given by $q_{7.6}=-q_{1}-q_{2}$, with the cooling rate for each subband $q_{i}$ computed 


$$
\begin{aligned}
q_{i}(r)=2 \pi n_{a}(\bar{\sigma} \Delta v)\left(-J(r)\left(\Gamma_{f 4}\left(r, r_{0}\right)+\Gamma_{f 4}\left(r, r_{t}\right)\right)\right. \\
+\int_{\Gamma_{f 4}\left(r, r_{0}\right)}^{1}\left(J\left(r^{\prime}\right)-J(r)\right) d \Gamma_{f 4}\left(r, r^{\prime}\right) \\
\left.+\int_{\Gamma_{f 4}\left(r, r_{t}\right)}^{1}\left(J\left(r^{\prime}\right)-J(r)\right) d \Gamma_{f 4}\left(r, r^{\prime}\right)\right)
\end{aligned}
$$

where $\bar{\sigma} \Delta v$ is the band strength of subband i. I have assumed there are no other sources of radiation in this band from the upper or lower boundaries of the atmosphere. The source function $J(r)$ is related to the cooling rate and the blackbody rate through

$$
J(r)=\frac{q_{i}(r)}{4 \pi n_{a}(\bar{\sigma} \Delta v) \phi_{4}}+B_{\bar{v}}(T)
$$

where $B_{\bar{v}}(T)$ is the Planck blackbody function evaluated from the local temperature for the line center wavenumber $\bar{v}$ of each subband. Eqns. (4.19) and (4.20) can be expressed in matrix vector form as

$$
\begin{gathered}
q_{i}=C J \\
J=E q_{i}+B
\end{gathered}
$$

Where $\boldsymbol{q}, \boldsymbol{J}$, and $\boldsymbol{B}$ are vectors with element being the cooling, source, and Planck blackbody rate, respectfully, at each level, and $\boldsymbol{C}$ is the Curtis matrix computed by the methods of Zhu (1990), and $\boldsymbol{E}$ is a diagonal matrix with the $\mathrm{j}^{\text {th }}$ diagonal element evaluated at level $r_{j}$ 


$$
[\boldsymbol{E}]_{j, j}=\left[\frac{1}{4 \pi n_{a}(\bar{\sigma} \Delta v) \phi_{4}}\right]_{r_{j}}
$$

From the coupled equations (4.21) and (4.22), I solve for $\boldsymbol{q}_{\boldsymbol{i}}$ by eliminating $\boldsymbol{J}$ in Eq. (4.22), obtaining

$$
\boldsymbol{q}_{i}=(\boldsymbol{I}-\boldsymbol{C E})^{-1}(\boldsymbol{C B})
$$

Where $\boldsymbol{I}$ is the identity matrix. Since $\boldsymbol{C}$ is a dense matrix (i.e., not a sparse matrix), solving for $\boldsymbol{q}_{\boldsymbol{i}}$ requires solving a dense linear system. This is accomplished using the DGESV routine from LAPACK, libraries are available on most machines.

\subsubsection{CO Rotational Cooling}

The carbon monoxide rotational levels should be in LTE down to pressures $\sim 10^{-5} \mu \mathrm{bar}$ (Strobel et al., 1996) and apply, therefore, to the majority of the atmosphere. Therefore, I can approximate the source function by $J(r)=B_{\bar{v}}(T)$, and can calculate the cooling for each CO rotational line directly from Eq. (4.19) as $\boldsymbol{q}_{\boldsymbol{c o}}=-\boldsymbol{C} \boldsymbol{B}$ using the matrix notation of the previous section. I only consider the 35 most significant lines (those with a strength greater than $1 \%$ of the strongest line) over the wavenumber range $3-134 \mathrm{~cm}^{-1}$. The lines are treated individually since they are well separated and the Planck blackbody function changes significantly over the wavenumber range. For the band escape function, the Doppler line escape function is used

$$
\Gamma_{D}(x)=\frac{2}{\sqrt{\pi}} \int_{0}^{\infty} e^{-t^{2}} E_{2}\left(x e^{-t^{2}}\right) d t
$$

where $\mathrm{x}$ is the optical thickness given by 


$$
x=\left|\int_{r}^{r \prime} \frac{n_{a}\left(z^{\prime \prime}\right) s(T)}{\sqrt{\pi} \alpha_{D}} d z^{\prime \prime}\right|
$$

In Eq. (4.26), $s(T)$ is the given line strength adjusted for temperature, and $\alpha_{D}$ is the Doppler half width. The line center $\bar{v}$, line strength $s(T)$, at the reference temperature $T_{s}=296 \mathrm{~K}$ are given. The net $\mathrm{CO}$ cooling rate is given by summing the contributions of the individual lines.

\subsubsection{UV Heating}

As I described earlier for the thermosphere model, heating in the upper atmosphere is due to the absorption of UV radiation. The energy flux for a plane-parallel atmosphere is given by Beer-Lambert's Law

$$
F(r)=\frac{1}{2} \mu F^{\infty} \exp \left(-\sigma_{a} N_{a} / \mu\right)
$$

where $F^{\infty}$ is the incoming energy flux, the geometric factor $\mu=\cos \theta$ to accommodate a slant incoming path, $\sigma_{a}$ is the absorber's cross section, and $N_{a}$ is the absorber's vertical columns density. The $1 / 2$ approximates the hemispherical averaging due to the planet's absorption cross-sectional area of $\pi r^{2}$ heating a hemisphere with an area $2 \pi r^{2}$. The heating rate per volume $q$ is then given by

$$
q(r)=\frac{d F}{d r}=\frac{1}{2} \sigma_{a} n_{a} F^{\infty} \exp \left(-\sigma_{a} N_{a} / \mu\right)
$$


Here $n_{a}$ is the absorber's local density. I use a diffusivity factor of $\eta=\frac{1}{\mu}=2$ to approximate the global averaging based on Apruzese (1980) in which a diffusivity factor of 2.07 was found to be optimal for intensity based heating calculations. Strobel et al. (1996) demonstrated that this diffusivity best approximated the hemispherical averaging in Eq. (4.3) near to the depth at which the optical depth is about one, while a larger value is better for smaller optical depths. Since most of the heat in Eq. (4.15) is deposited near where the optical depth is one, I find using $\mu=0.5$ to be sufficient for our interests.

The values for the UV and EUV cross-section and energy fluxes for methane and nitrogen, respectively, used in the thermosphere model given in Krasnopolsky (1999). He assumed that all the radiation in the UV range $80-140 \mathrm{~nm}$ was absorbed by methane with a heating efficiency of 0.5 , and all the radiation in the EUV range $0-80 \mathrm{~nm}$ was absorbed by nitrogen with a heating efficiency of 0.25 . The cross section and globally average energy fluxes (in our convention) are given in Table (1) for the cases of minimum, mean, and maximum total solar UV irradiance.

\begin{tabular}{|c|c|c|c|}
\hline \multicolumn{2}{|c|}{} & $0-80 \mathrm{~nm}(\mathrm{~N} 2)$ & $80-140 \mathrm{~nm}(\mathrm{CH} 4)$ \\
\hline \multicolumn{2}{|c|}{$\sigma(10-17 \mathrm{~cm} 2)$} & 0.913 & 1.8 \\
\hline \multirow{3}{*}{$\begin{array}{c}\frac{1}{2} F^{\infty} \\
\left(10^{-3} \mathrm{erg} \mathrm{cm}^{-2} \mathrm{~s}^{-1}\right)\end{array}$} & Min & 0.34 & 1.65 \\
\cline { 2 - 4 } & Mean & 0.58 & 2.80 \\
\cline { 2 - 4 } & Max & 0.98 & 4.80 \\
\hline
\end{tabular}

Table 6: UV and EUV absorption cross section and energy flux values from Krasnopolsky (1999)

I instead used measured UV and EUV solar spectra and cross section available from NASA Thermosphere Ionosphere Mesosphere Energetics and Dynamics (TIMED) Solar EUV Experiment (SEE). In particular I used the Level 3 Spectra, which is solar spectral irradiance (SSI) combined from two experiments to cover a spectral range from 0 to 
$195 \mathrm{~nm}$ in $1 \mathrm{~nm}$ bins (http://lasp.colorado.edu/lisird/see/). The values are corrected so that the irradiance value is scaled to 1 AU from the sun, so I further scale it by Pluto's orbital radius from the sun. The following is a plot of a sample SSI, along with cross sections for nitrogen and methane.
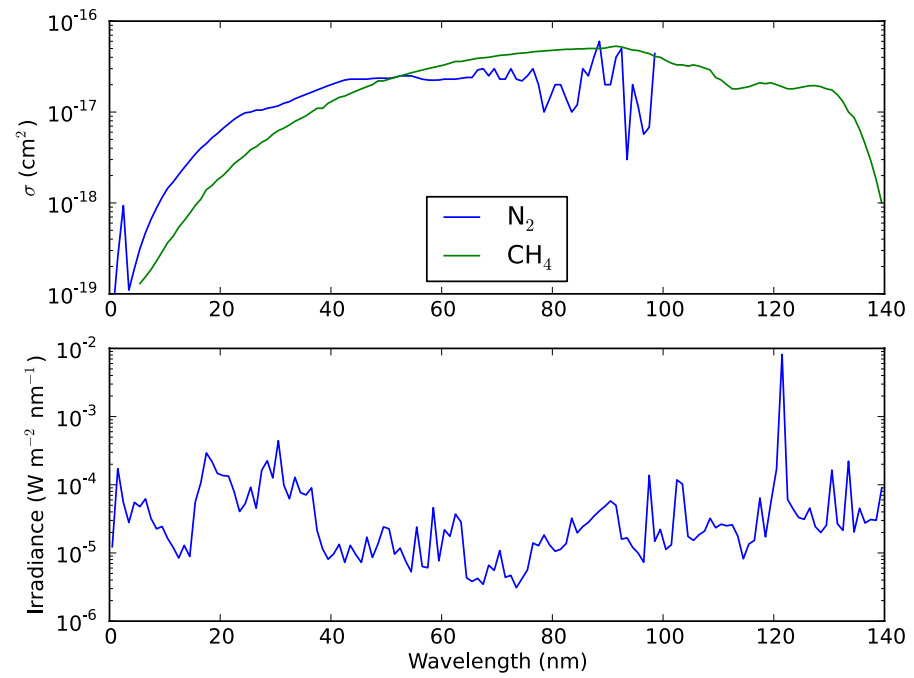

Figure 4-1: Absorption cross-section for nitrogen and methane from 0 to $140 \mathrm{~nm}$. Solar spectral irradiance for the same range from March 30, 1999 retrieved from http://lasp.colorado.edu/lisird/see/.

The absorption cross section of methane decreases significantly above $140 \mathrm{~nm}$, while the absorption cross section for nitrogen decreases rapidly above $100 \mathrm{~nm}$. The division of the spectra by Krasnopolsky (1999) can be justified by this lack of nitrogen absorption above $100 \mathrm{~nm}$. Also I justify the omission of heating by methane at shorter wavelengths, since nitrogen's cross section is comparable if not larger and nitrogen is the dominant species. Therefore, in this range the heating rate for nitrogen computed from Eq. (4.30) will be much larger than that of methane. The peak in the SSI at $121.6 \mathrm{~nm}$ due to the Lymanalpha radiation. 
To save computation, I divide the UV spectrum into 3 sub-ranges: 0-40nm, 40$100 \mathrm{~nm}$, and $100-140 \mathrm{~nm}$. Nitrogen has zero absorption in the highest range, and the methane absorption will be almost entirely Lyman-alpha radiation. The two lower ranges are separated based on the order of magnitude difference in the SSI at $40 \mathrm{~nm}$. The effective cross sections, $\bar{\sigma}_{a, i}$, and energy fluxes, $\bar{F}_{i}$, for each wavelength sub-range $R_{i}$ by summing over wavelengths $\lambda_{j}$ with bin size $\Delta \lambda=1 \mathrm{~nm}$ in each sub-range

$$
\begin{gathered}
\bar{F}_{i}=\sum_{j \in R_{i}} F_{j} \Delta \lambda \\
\bar{\sigma}_{a, i}=\frac{1}{\bar{F}_{i}} \sum_{j \in R_{i}} F_{j} \sigma_{a, j} \Delta \lambda
\end{gathered}
$$

The effective energy flux is the total energy flux in each range, and the effective cross section is the weighted average of the cross section in range weighted by the energy flux at each wavelength. Since both species may absorb in each sub-range, the optical depth is the sum of the optical depths due to each species, and the heating rate is the sum of the heating rates due to each species. Therefore the heating rate for species $a$ in the sub-range $R_{i}$ is given by

$$
q_{a, i}(r)=\frac{1}{2} \sigma_{a, i} n_{a} \epsilon_{a} \bar{F}_{i} \exp \left(-\sum_{a} \bar{\sigma}_{a, i} N_{a} / \mu\right)
$$

Here I use fixed heating efficiencies, $\epsilon_{a}$, of 0.5 and 0.25 for methane and nitrogen, respectively, as in Krasnopolsky (1999). Using the effective cross section and effective flux reduces the number of computations and the total flux absorbed is the conserved. 
Furthermore, since the effective cross section favors parts of the spectrum with high fluxes, the shape of the heating profile is well approximated.

To compare to Krasnopolsky (1999) I first list the effective cross sections and effective energy flux I use to compute the absorption for the same sub-range divisions for solar minimum conditions.

\begin{tabular}{|c|c|c|c|}
\hline \multicolumn{2}{|c|}{} & $0-80 \mathrm{~nm}$ & $80-140 \mathrm{~nm}$ \\
\hline \multirow{2}{*}{$\bar{\sigma}_{a}\left(10^{-17} \mathrm{~cm}^{2}\right)$} & $\mathrm{N}_{2}$ & 1.06 & 0.11 \\
\cline { 2 - 4 } & $\mathrm{CH}_{4}$ & 0.85 & 2.02 \\
\hline \multirow{2}{*}{$\begin{array}{c}\frac{1}{2} \epsilon_{a} \bar{F} \\
\left(10^{-3} \mathrm{erg} \mathrm{cm}^{-2} \mathrm{~s}^{-1}\right)\end{array}$} & $\mathrm{N}_{2}$ & 0.29 & 1.07 \\
\cline { 2 - 4 } & $\mathrm{CH}_{4}$ & 0.58 & 2.13 \\
\hline
\end{tabular}

Table 7: Effective cross section and effective energy fluxes computed from the SSI for March 30, 2003 (a solar minimum like case) scaled to $30 \mathrm{AU}$ for the sub-ranges $0-80 \mathrm{~nm}$ and $80-140 \mathrm{~nm}$.

The values for effective cross section and effective energy flux for nitrogen in the 0 $80 \mathrm{~nm}$ range from Table 2 compares well with the corresponding values for solar minimum case from Table 1, with the differences in both quantities being $\sim 15 \%$. Similarly for methane in the $80-140 \mathrm{~nm}$ range, the differences are $\sim 20 \%$ for the effective energy flux and $\sim 10 \%$ for effective cross section. To judge the relative contribution of nitrogen and methane to the net heating rate I compare the product $\frac{1}{2} \sigma_{N 2, i} \epsilon_{N 2} \bar{F}_{i}$ to $\frac{1}{2} \sigma_{C H 4, i} \chi_{C H 4} \epsilon_{C H 4} \bar{F}_{i}$; this is the relative value for heating of each species assuming $\chi_{C H 4}=0.01$. For the $0-80$ range I get 0.31 for nitrogen and 0.005 for methane, hence methane can easily be ignored in this region as it is $\sim 60$ times weaker. For the $80-140 \mathrm{~nm}$ sub-range I get 0.12 for nitrogen and 0.043 for methane, which are comparable, hence, nitrogen should not be ignored in this range. If the range were adjusted to $100-140 \mathrm{~nm}$ then the nitrogen contribution would be negligible. 


\begin{tabular}{|c|c|c|c|c|c|c|c|}
\hline & \multicolumn{2}{|c|}{$0-40 \mathrm{~nm}$} & \multicolumn{2}{c|}{$40-100 \mathrm{~nm}$} & \multicolumn{2}{c|}{$100-140 \mathrm{~nm}$} \\
\hline & $\mathrm{N}_{2}$ & $\mathrm{CH}_{4}$ & $\mathrm{~N}_{2}$ & $\mathrm{CH}_{4}$ & $\mathrm{~N}_{2}$ & $\mathrm{CH}_{4}$ \\
\hline \multicolumn{2}{|c|}{$\bar{\sigma}_{a}\left(10^{-17} \mathrm{~cm}^{2}\right)$} & 0.81 & 0.37 & 2.27 & 4.03 & 0.00 & 1.88 \\
\hline \multirow{2}{*}{$\begin{array}{c}\frac{1}{2} \epsilon_{a} \bar{F} \\
\left(10^{-3} \mathrm{erg} \mathrm{cm}^{-2} \mathrm{~s}^{-1}\right)\end{array}$} & Min & 0.24 & 0.48 & 0.10 & 0.20 & - & 2.03 \\
\cline { 2 - 8 } & Mean & 0.49 & 0.97 & 0.15 & 0.31 & - & 2.76 \\
\cline { 2 - 8 } & Max & 0.68 & 1.35 & 0.17 & 0.34 & - & 3.58 \\
\hline
\end{tabular}

Table 8: Values for effective cross section and effective solar energy flux given in the 3 sub-ranges for case of solar minimum, mean, and maximum-like conditions.

Values for effective cross section and effective energy flux for 3 chosen cases are given in Table 3. The dates of March 30, 2009, March 1, 2003, and February 8, 2002 are used for a solar minimum, medium, and maximum like conditions based on the intensity of the Lyman-alpha line and the data available. The SSI data sets used are not available before February 1, 2002, so the solar maximum case is the largest heating rate available.

Our treatment of constant heating efficiencies can produce numerical instabilities when modeling the upper thermosphere and into the exosphere. The efficiency in the upper thermosphere decreases with increasing altitude since the transfer of absorbed energy to kinetic energy becomes inefficient at very low densities. This can be calculated in detail using photochemical models (Yelle, 2003; Tian, 2008). I adopt a simple form for the decrease in efficiency

$$
\epsilon_{a}(r)=\epsilon_{a}\left(1-e^{-K n(r)}\right)
$$




\subsection{Hybrid Model}

I use the fluid-Jeans and fluid-DSMC models introduced in Thermosphere Modeling chapter, but include the new heating methods and updated the simulation parameters in order to represent the larger simulation domain. In Table 4, I list the parameter for our base case. I will vary the lower boundary pressure $p_{0}$, the methane and carbon monoxide mixing ratios $\chi_{C H 4}$ and $\chi_{C O}$, and the Pluto-Sun distance $r_{P S}$ from these values to study their influence on the solution.

\begin{tabular}{|c|l|}
\hline Parameter & Value \\
\hline$r_{0}$ & $1180 \mathrm{~km}$ \\
\hline$T_{0}$ & $37.0 \mathrm{~K}$ \\
\hline$p_{0}$ & $2.0 \mathrm{~Pa}$ \\
\hline$\chi_{C H 4}$ & 0.0025 \\
\hline$\chi_{C O}$ & 0.0005 \\
\hline$\kappa_{0}$ & $5.63 \times 10^{-5} \mathrm{Jm}^{-1} \mathrm{~s}^{-1}$ \\
\hline$\omega$ & 1.12 \\
\hline$r_{P S}$ & $32.9 \mathrm{AU}$ \\
\hline
\end{tabular}

Table 9: Base case parameters for full atmosphere model.

Pluto's radius and surface temperature are fixed at $1180 \mathrm{~km}$ and $37 \mathrm{~K}$ following the best-fit solution of Zalucha et al. (2011). The new heating model limits the number of radial grid points, $N_{r}$, since the computations required are mostly time intensive. In particular the methane $7.6 \mathrm{~mm}$ line requires the solution to a linear system, which requires $O\left(N_{r}^{3}\right)$ computation time. I use a radial grid step-size of $d r_{0}=1 \mathrm{~km}$, and increase the step-size at each step according to $d r_{n+1}=1.003 \times d r_{n}$ as this provides better resolution 
near the lower boundary than the scheme used in the thermosphere model. Using 242 radial steps, $N_{r}$, gives an upper boundary $r_{t}=13580 \mathrm{~km}$, and using $N_{r}=252$ gives an upper boundary $r_{t}=17850 \mathrm{~km}$. While 242 radial points is sufficient for most cases, 252 radial points are necessary for some of the higher heating cases when the exobase radius is greater than $13580 \mathrm{~km}$.

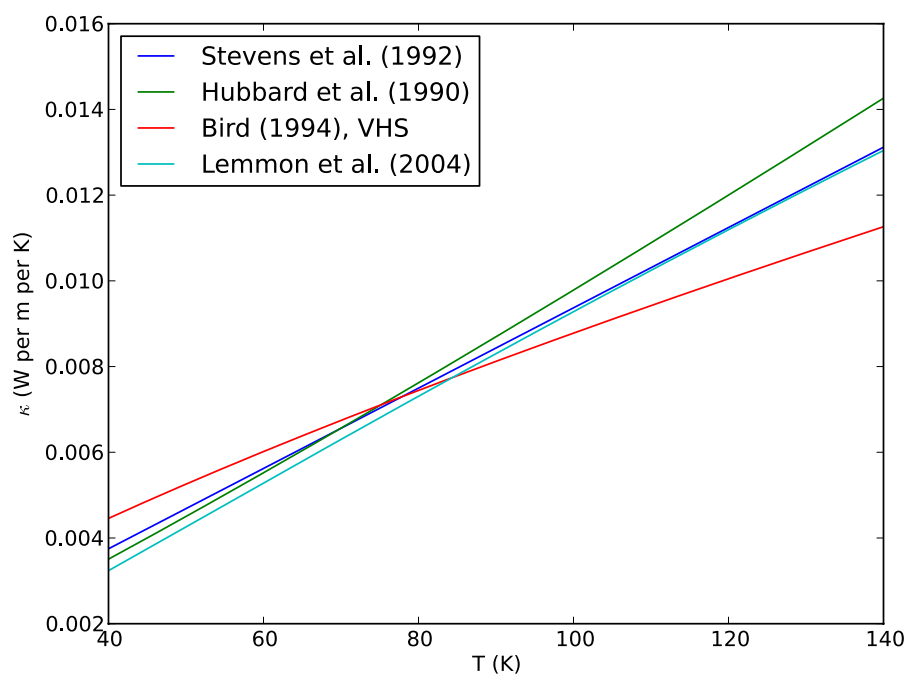

Figure 4-2: Conductivity for nitrogen for a temperature range relevant to Pluto. Stevens et al. (1992), Hubbard et al. (1990), and Bird (1994) are power laws, while Lemmon et al. (2004) is a detailed parameterization.

I have changed the conductivity from that used in the Thermosphere Modeling chapter. Lemmon et al. (2004) gave a parameterization of the viscosity of nitrogen that closely approximates measurements values down to temperatures $60 \mathrm{~K}$. From this I found a best-fit power law for the nitrogen's conductivity of $5.80 \times 10^{-5} T^{1.10}$ for the temperature range 40-140K. In Figure (4-2) I plotted this parameterization along with the other conductivities considered in models of Pluto's atmosphere. Experiments performed using these different forms of conductivity found that the differences in solutions were small, which I attribute to the values of the conductivity being similar in this temperature range. I adopt the values from Hubbard et al. (1990) for a nitrogen/methane mixture since 
this has been used in previous modeling (Strobel et al., 1996; Zalucha et al., 2011) and the viscosity exponent is close to the best-fit power law to Lemmon's conductivity.

The time stepping procedure for the fluid-Jeans model is described in Chapter 2. The time step is set to between $0.1-1$ days $\left(\sim 10^{4}-10^{5} \mathrm{~s}\right)$ depending on solar activity, with the smaller step required for the higher solar heating cases, and when the larger upper boundary is used. To save on computation time, the heating rates are recalculated every 10 time steps. Convergence is monitored by computing the value $\frac{\Delta t}{N_{r}} \sqrt{\sum \Delta T_{i}^{2}}$, which is the 2-norm of the change in temperature scaled by time step and number of radial steps. About 100,000 times steps (with 10,000 heating recalculations) are necessary to obtain a consistent solution, with convergence value smaller than $10^{-5}$.

\subsubsection{Fluid-Jeans solution}

In Figure (4-5), it is seen that UV heating significantly affects the temperature structure of upper atmosphere (above $r=1.5 r_{p}$ ). The two peaks, due to methane and nitrogen UV absorption, can be seen with the peak methane absorption occurring below the peak nitrogen absorption due to methane's small mixing ratio. Unlike in the results for the thermosphere, the UV heating does not create a large temperature maximum above $r=1.2 r_{0}$. With the exception of the case of solar maximum UV heating, the temperature monotonically decreases with altitude above the peak temperature of $\sim 133 \mathrm{~K}$ at $r \sim 1.1 r_{p}$. In the case of solar maximum UV heating, there is a small temperature maximum associated with the methane UV absorption. 


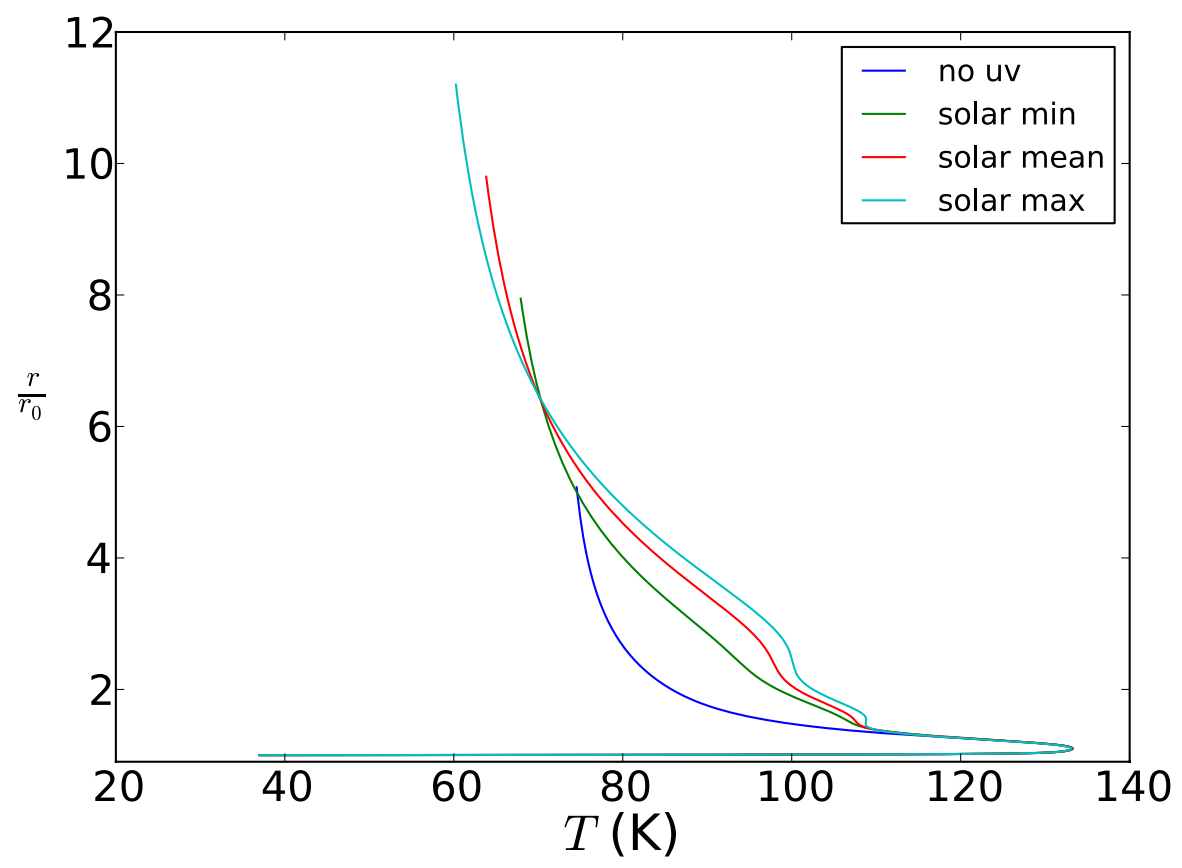

Figure 4-3: Temperature profiles of solutions to fluid-Jeans full atmosphere model.

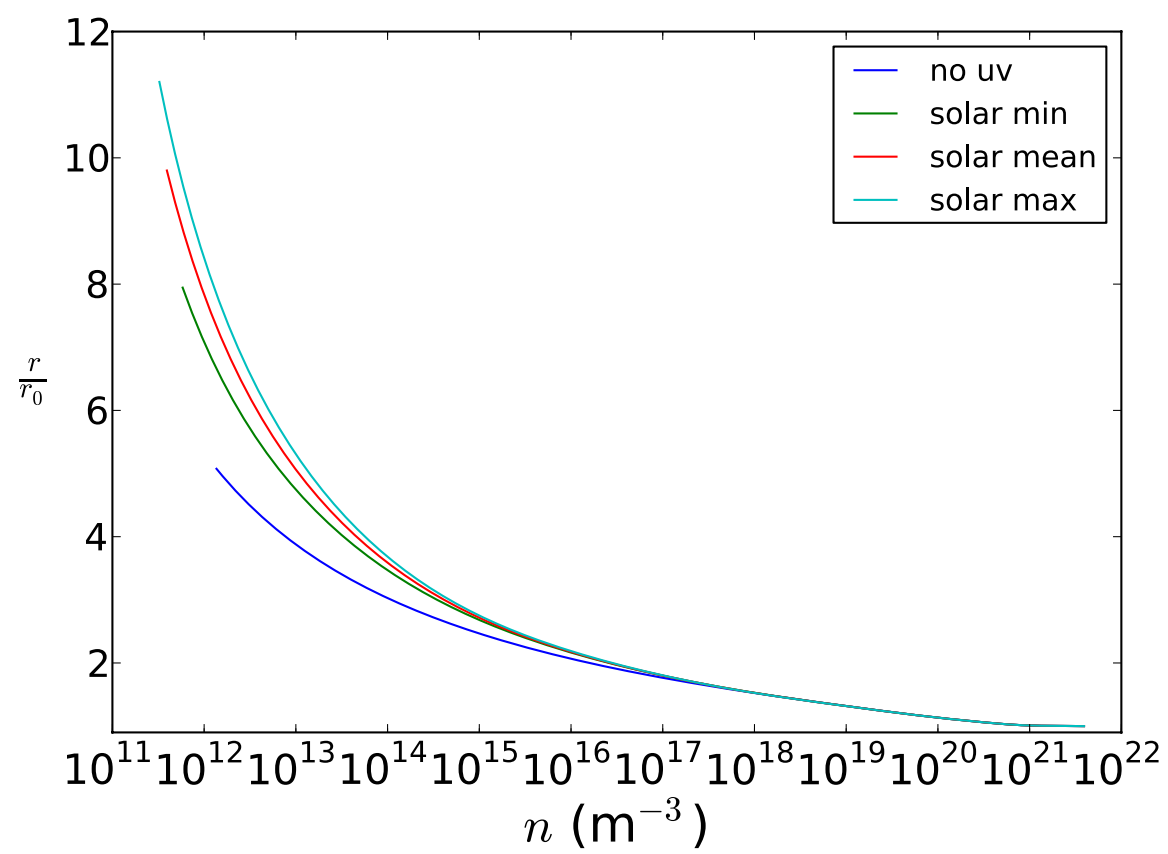

Figure 4-4: Density profile for Fluid-Jeans solutions.

Similar to the thermosphere results, there is an order of magnitude increase in escape rate when UV heating is included. The total escape rate is in the range $\sim 2$ to 
$\sim 5 \times 10^{27} \mathrm{~s}^{-1}$, which is consistent estimate of the total escape rate (Krasnopolsky, 1999; Strobel, 2008; Tucker et al., 2011; Erwin et al., 2013).

\begin{tabular}{|c|r|r|r|r|}
\hline & NO UV & MIN & MED & MAX \\
\hline$\phi\left(10^{27} \mathrm{~s}^{-1}\right)$ & 0.40 & 2.20 & 3.71 & 5.10 \\
\hline$E_{\text {esc }}\left(10^{-3} \mathrm{eV}\right)$ & 13.6 & 12.3 & 11.4 & 10.7 \\
\hline$r_{x} / r_{0}$ & 5.2 & 8.1 & 9.8 & 11.4 \\
\hline$n_{x}\left(10^{12} \mathrm{~m}^{-3}\right)$ & 1.20 & 0.54 & 0.39 & 0.31 \\
\hline$Q_{U V}\left(10^{7} \mathrm{~W}\right)$ & - & 6.1 & 9.7 & 13.0 \\
\hline
\end{tabular}

Table 10: Values from the solutions from the fluid-Jean full atmospheric model.

The ratio of escape rate to UV heating is $0.36,0.38$, and $0.39 \times 10^{20} \mathrm{~s}^{-1} \mathrm{~W}^{-1}$ for solar minimum, mean, and maximum, respectfully. Hence, the escape rate increases roughly linearly with UV heating and may be estimated as energy limited due to UV heating. The energy limited escape rate due to UV heating from an atmospheric level of escape $r_{e}$ is expressed as $\phi_{E L} \sim Q_{U V} / U\left(r_{e}\right)$ (Lammer et al., 2009; Johnson et al. 2013). From the solution I estimate $r_{e}=1.4 r_{0}$ as the position where UV heating becomes larger than the IR heating. Then the energy limited rate is $\sim 2.4,3.8,5.0 \times 10^{27}$ for solar minimum, mean, and maximum cases respectfully. Comparing to the actual escape rate from Table 5, the energy limited rate is a good estimate of escape rate even in the full atmosphere model.

The temperature and density structure of Pluto's lower atmosphere are only slightly affected by the change in adiabatic cooling due to solar UV activity. The peak temperature increases by only a fraction of a degree by the introduction of UV heating. The methane IR heating is most dependent on the column density and dominates the 
lower atmosphere structure. The $\mathrm{N}_{2}$ column density at the surface only changes by $0.02 \%$ over the range solar activity described. Below $r=1.5 r_{0}$ the difference between the densities of the UV solutions and the no UV solution is less than 5\%, while below $r=1.2 r_{0}$ the difference in density is less than $0.5 \%$. Stellar occultation measurements of Pluto's atmosphere are only sensitive to atmospheric below 1500km (Elliot et al., 1989). The best-fit solutions of Zalucha et al. (2011) have largest residuals between 10\% to 50\% depending on occultation event, so the UV activity may have a small affect if this model were used to fit stellar occultation curves.

\begin{tabular}{|r|r|c|c|c|}
\hline Energy loss mechanism $\left(10^{8} \mathrm{~W}\right)$ & NO UV & MIN & MED & MAX \\
\hline Escape @ $r_{x}$ & 0.01 & 0.04 & 0.07 & 0.09 \\
\hline Conduction @ $r_{0}$ & 8.46 & 8.40 & 8.34 & 8.29 \\
\hline Adiabatic cooling @ $r_{0}$ & 0.13 & 0.71 & 1.21 & 1.66 \\
\hline
\end{tabular}

Table 11: Comparison of energy loss mechanism through upper and lower boundaries.

The temperature gradient at the surface changes by only $2 \%$ due to UV activity, and the escape rate in the presence of significant UV heating has a non-negligible contribution to the energy conservation in the atmosphere. In Table 11, the three mechanisms by which the atmosphere can dissipate the net radiative heating are listed: escape to space, adiabatic cooling at the surface, and conduction to the surface. With UV heating included, the adiabatic cooling is between $10 \%$ and $20 \%$ as efficient as conduction in cooling the atmosphere. The energy carried off by escaping molecule is small compared to the cooling at the surface.

\begin{tabular}{|c|r|r|r|r|}
\hline Integrated Heating $\left(10^{8} \mathrm{~W}\right)$ & NO UV & MIN & MED & MAX \\
\hline$Q_{\text {net }}$ & 9.2 & 9.8 & 10.2 & 10.6 \\
\hline
\end{tabular}




\begin{tabular}{|c|r|r|r|r|}
\hline$Q_{2.3}$ & 22.8 & 22.8 & 22.8 & 22.7 \\
\hline$Q_{3.3}$ & 3.6 & 3.6 & 3.6 & 3.6 \\
\hline$Q_{7.6}$ & 10.7 & 10.7 & 10.6 & 10.5 \\
\hline$Q_{C O}$ & 6.5 & 6.6 & 6.6 & 6.6 \\
\hline$Q_{U V, C H 4}$ & 0.0 & 0.41 & 0.56 & 0.74 \\
\hline$Q_{U V, N 2}$ & 0.0 & 0.23 & 0.47 & 0.66 \\
\hline
\end{tabular}

Table 12: The integrated heating rates from the different heating mechanisms.

\subsubsection{Heating Discussion}

The IR heating/cooling models for methane and carbon monoxide are the same as Zalucha et al. (2011), which is itself an update of Strobel et al. (1996). Yet there are some significant changes to the results of the heating/cooling models when I implemented the full atmosphere model with new lower boundary parameters. In Figure (4-3), the heating and cooling rates are given for the converged solution of the fluid-Jeans model with solar minimum UV activity.

In Strobel et al. (1996) and Zalucha et al. (2011), the upper boundary altitude was set to $700 \mathrm{~km}$ and $600 \mathrm{~km}$, respectively, which corresponds to $r=1.6 r_{0}$ and $r=1.5 r_{0}$ respectively. But Figure (4-5) shows that this does not capture the full non-LTE behavior of the $2.3 \mu \mathrm{m}$ or $3.3 \mu \mathrm{m}$ bands when the thermosphere is included in the simulations. The $(\mathrm{V}-\mathrm{V})$ transition efficiently heats the atmosphere at these altitudes, which requires the atmosphere to be thin in the respective bands $\left(v_{2}\right.$ or $\left.v_{3}\right)$ as well as the $v_{4}$ band. Note that this heating occurs at greater altitude in the full atmospheric model since the methane mixing ratio is a factor $\sim 10$ smaller. In Strobel et al. (1996), this heating occurred 
$\sim 300 \mathrm{~km}$ below the upper boundary, and I find the scale height to be $\sim 200 \mathrm{~km}$ at this altitude. Hence, there might have been some error in computing the column density of methane and, hence, the escape functions ( $\Gamma^{\prime}$ 's) used to compute the heating efficiencies at these altitudes.

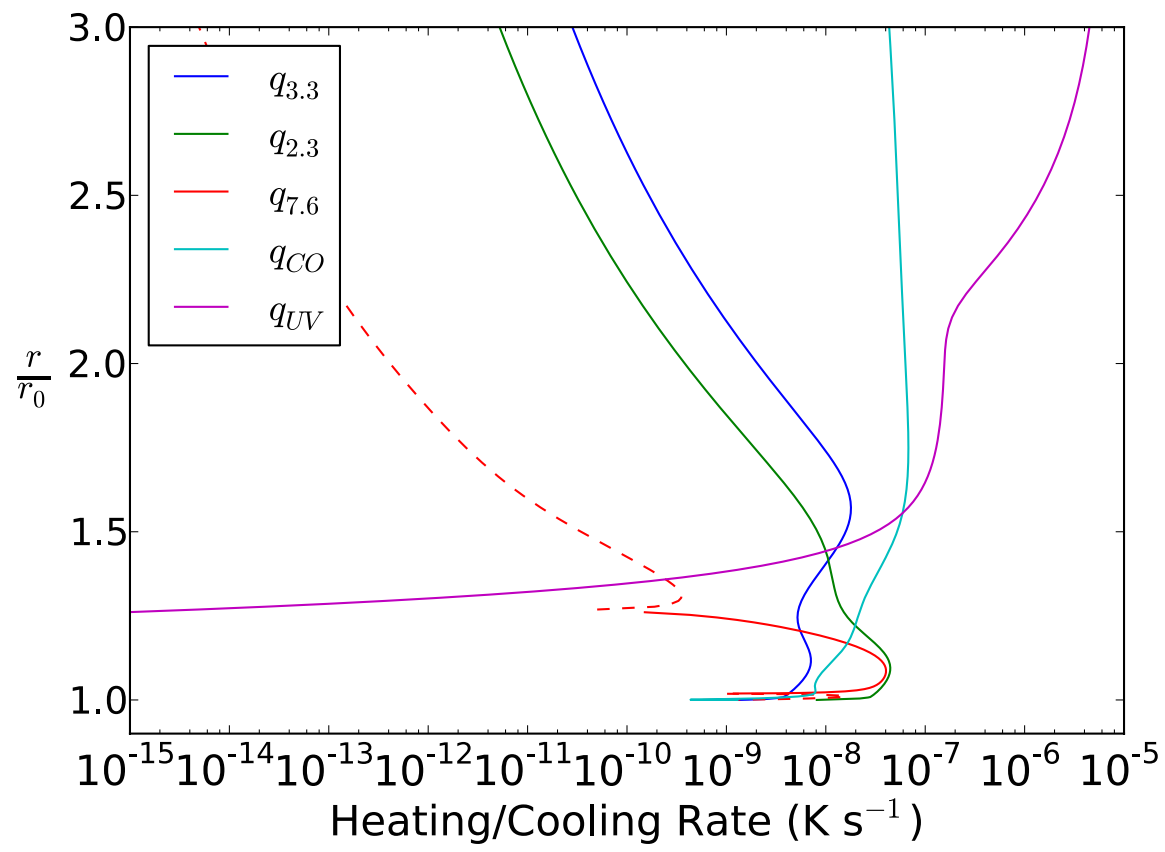

Figure 4-5: Heating and cooling rates in the lower atmosphere for the fluid-Jeans solution with solar minimum UV activity. By the sign convention for the cooling rates, a solid line denotes local cooling and a dotted denotes local heating.

Strobel et al. (1996) showed the high temperatures in the middle atmosphere heated the lowest $\sim 20 \mathrm{~km}$ via the $7.6 \mu \mathrm{m}$ band. Now with an extended, cold atmosphere above the stratosphere, a small amount of this heat is absorbed in the upper atmosphere. However, above $r=1.5 r_{0}$ this heating is negligible compared to the UV heating and $\mathrm{CO}$ cooling as Figures (4-4) and (4-5) show.

Strobel et al. (1996) states that the CO rotational cooling should be in LTE down to pressures $\sim 10^{-5} \mu$ bar. The pressure drops below $10^{-5} \mu$ bar above $r=2.7 r_{0}$. At this 
altitude the $\mathrm{CO}$ rotational cooling is $\sim 1 \%$ of the UV heating, becoming less significant with increasing altitude. Therefore the non-LTE effects of the CO rotational cooling are not included.

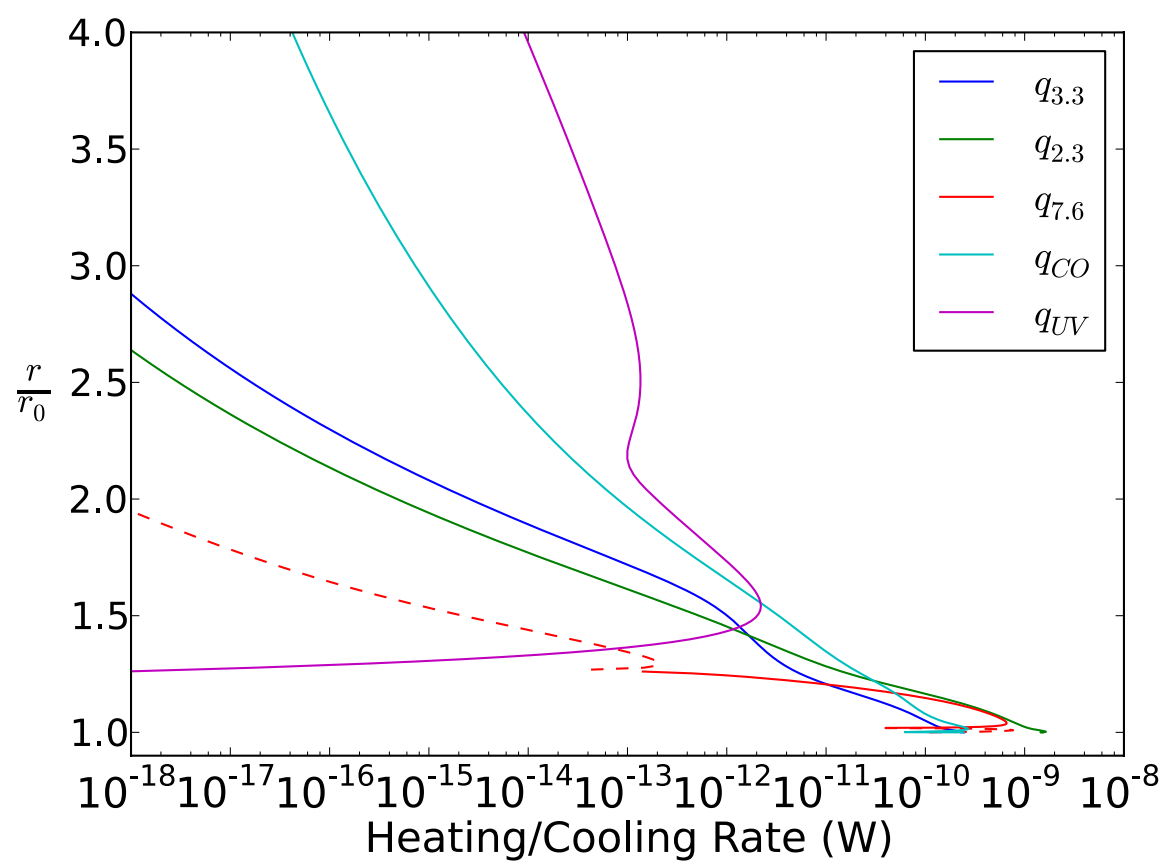

Figure 4-6: Heating and cooling rates for the fluid-Jeans solution with solar minimum UV activity. By the sign convention for the cooling rates, a solid line denotes local cooling and a dotted denotes local heating.

The methane UV heating exceeds the methane IR heating at $r \sim 1.4 r_{0}$, and exceeds the carbon monoxide cooling above $r \sim 1.6 r_{0}$. Hence, the UV heating contribution to the atmospheric structure starts near the upper boundary of Strobel et al. (1996) and Zalucha et al. (2011). In the thermosphere models of Strobel (2008), Tucker et al. (2012), and Erwin et al. (2013), the methane IR heating was simply parameterized. But the methane IR heating has a non-trivial non-LTE behavior above the lower boundary of $r \sim 1.2 r_{0}$. By modeling the full atmosphere, I accommodate the overlapping of the methane IR heating and UV heating models. 
The peak UV heating occurs at $\sim 1.6 r_{0}$ and $\sim 2.6 r_{0}$ for methane and nitrogen, respectfully. This is close to the altitude of unit optical depths using the UV cross sections from Table (8). The UV heating peaks do not move significantly with changes in the UV heating

\subsubsection{Fluid-DSMC Results}

Although I showed in Chapter 3 that the fluid-Jeans model is useful in predicting the escape rate for the full atmosphere, the fluid-DSMC model is necessary in order to correctly model the upper atmospheric structure. Using the same technique as in Tucker et al. (2012) and Erwin et al. (2013), I find a consistent fluid-DSMC solution to the full atmosphere for no UV heating and for solar minimum and solar mean heating cases. I set the Pluto-Sun distant to 32.9 AU so that the solar minimum and solar mean cases can be used as an approximate bounds for the NH encounter.

The DSMC model and iteration procedure are the same as in the Chapter 3, with the exception of the collisional parameters. The collisional cross-section viscosity exponent was changed to be consistent with the conductivity expression $5.80 \times 10^{-5} T^{1.10}$ used in the fluid region of the full atmosphere. The DSMC solution is started from the altitude where $K n \sim 0.1$, which is $3290,4970,5450 \mathrm{~km}$ for the no UV, solar minimum and solar mean cases, respectively. In each case, I obtained fluid-Jeans solutions for different values of the escape rate enhancement. In this way I rapidly narrowed in on a solution in which the escape rate between the fluid and DMSC domains were consistent. 


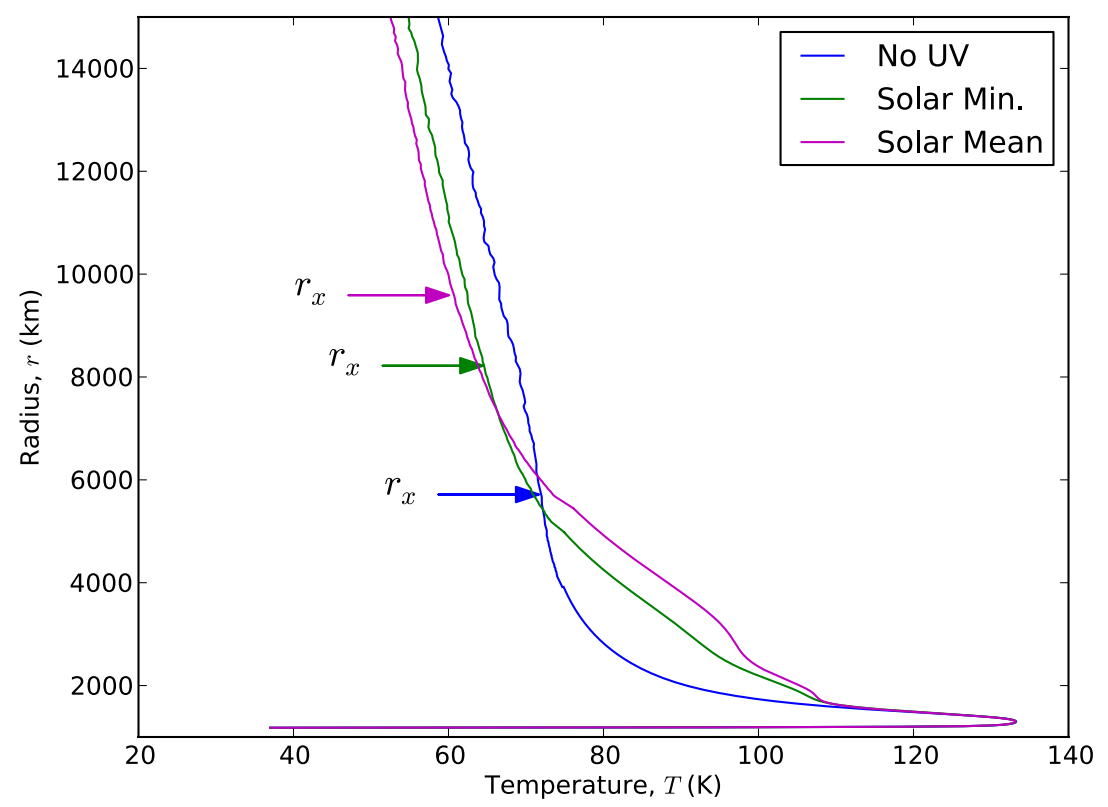

Figure 4-7: Temperature profiles from fluid-DSMC model of Pluto's full atmosphere.

\begin{tabular}{|c|r|r|r|}
\hline & \multicolumn{1}{|l|}{ No UV } & Solar Min. & Solar Mean \\
\hline$r_{x}(\mathrm{~km})$ & 5670 & 8220. & 9650. \\
\hline$T_{x}(\mathrm{~K})$ & 72.0 & 64.6 & 60.6 \\
\hline$n_{x}\left(\mathrm{~m}^{-3}\right)$ & $1.42 \cdot 10^{12}$ & $7.53 \cdot 10^{11}$ & $6.04 \cdot 10^{11}$ \\
\hline$\lambda$ & 7.2 & 5.5 & 5.0 \\
\hline$\phi\left(\mathrm{s}^{-1}\right)$ & $4.50 \cdot 10^{26}$ & $2.21 \cdot 10^{27}$ & $3.70 \cdot 10^{27}$ \\
\hline$E_{\text {esc }}(\mathrm{eV})$ & $11.8 \cdot 10^{-3}$ & $10.4 \cdot 10^{-3}$ & $9.82 \cdot 10^{-3}$ \\
\hline$\phi /\langle\phi\rangle_{J}$ & 2.1 & 2.4 & 2.5 \\
\hline
\end{tabular}

Table 13: Characteristic values from fluid-DSMC simulation of Pluto's full atmosphere.

The results of the fluid-DSMC models for Pluto's full atmosphere are presented in Figure (4-7) and Table (13). The escape rate calculated using the fluid-DSMC model is nearly the same as the fluid-Jeans results given in Table (10), but the exobase is 
considerably lower as the escape rate is enhanced. The exobase for solar minimum and mean is found to occur roughly halfway between Pluto and Charon.

Using the full atmosphere model, the temperature in the exosphere is surprisingly cooler as compared to that obtained in my earlier calculations in which I treated only the upper atmosphere. Consistent with this, Greaves et al. (2011) observed a CO emission feature which they suggested was indicative of an atmosphere at $\sim 50 \mathrm{~K}$ and appeared to detect the $\mathrm{CO}$ emission feature out to about $\sim 4500 \mathrm{~km}$. Since $\mathrm{CO}$ has a mass similar to $\mathrm{N}_{2}$, I expect it to have a similarly extended profile. Therefore, the fluid-DSMC description of the full atmospheric profile appears to be closer to the these observations than are the fluid-Jeans profiles of the full atmosphere or any of the profiles of the upper atmosphere calculated in Chapter 3.

The escape rate enhancement obtained in the full atmosphere fluid-DSMC model is between 2.1 and 2.5 times the Jeans rate as compared to the 1.6-2.3 enhancement given in Table (1) for the fluid-DSMC model of the upper atmosphere alone. Adapting the trend in enhancement shown in Volkov et al. (2011), the increased enhancement comes about because the Jeans parameter at the lower boundary of the DSMC domain is comparably lower.

\subsubsection{Surface Pressure, Mixing Ratio, and Orbital Distance}

I studied the sensitivity of the atmosphere to lower boundary parameters. Zalucha et al. (2011) found the stellar occultation were particularly sensitive to surface pressure and the 
methane mixing ratio. I used the solar minimum UV heating rates, and I held the enhancement in the Jeans escape rate fixed at 2 to approximate the DSMC escape rate.

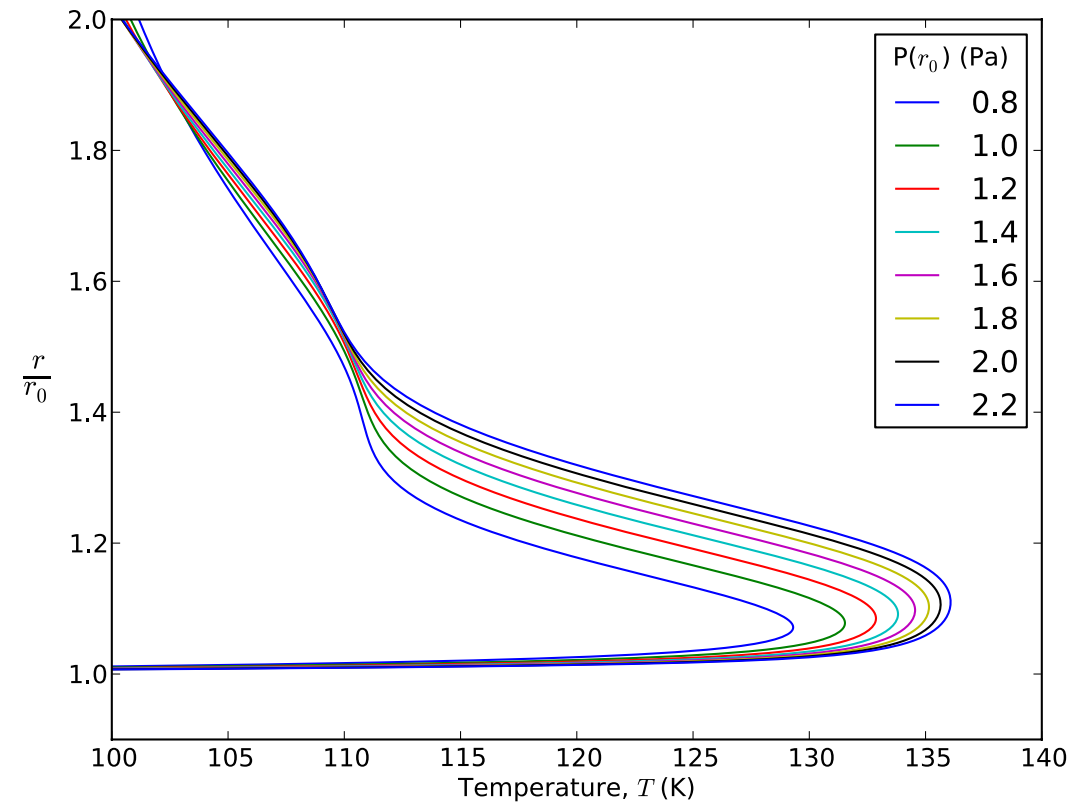

Figure 4-8: Temperature profiles in the lower atmosphere for surface pressures between 0.8 and 2.2Pa.

The surface pressure was found to have a significant effect on the $2.3 \mu \mathrm{m}$ and $3.3 \mu \mathrm{m}$ methane heating band as well as on the $7.6 \mu \mathrm{m}$ methane cooling band. The $2.3 \mu \mathrm{m}$ and $3.3 \mu \mathrm{m}$ bands are optically thick; therefore all their radiative energy is absorbed. By increasing the surface pressure, the energy is absorbed at a higher altitude.

In Figure (4-7) I give the temperatures in the lower atmosphere for several values of surface pressure between 0.8 and $2.2 \mathrm{~Pa}$. The peak temperature increases with surface pressure from $\sim 129-136 \mathrm{~K}$. The temperature gradient at the lower boundary likewise varies from $12-17 \mathrm{~K} \mathrm{~km}^{-1}$. This range of temperature gradients is at the low end of the values (10-30 $\left.\mathrm{K} \mathrm{km}^{-1}\right)$ that Stansberry et al. (1994) suggested might be responsible for an apparent kink in the temperature structure that was estimated from stellar occultation curves. 
The escape rate is seen to increase almost linearly over the range of reasonable surface pressures that I considered: $1.8-3.2 \times 10^{27} \mathrm{~s}^{-1}$. As the surface pressure increases the temperature above $r=2 r_{0}$ decreases due to the increase in adiabatic cooling, but the exobase radius increases from $6.1-8.4 r_{0}$.

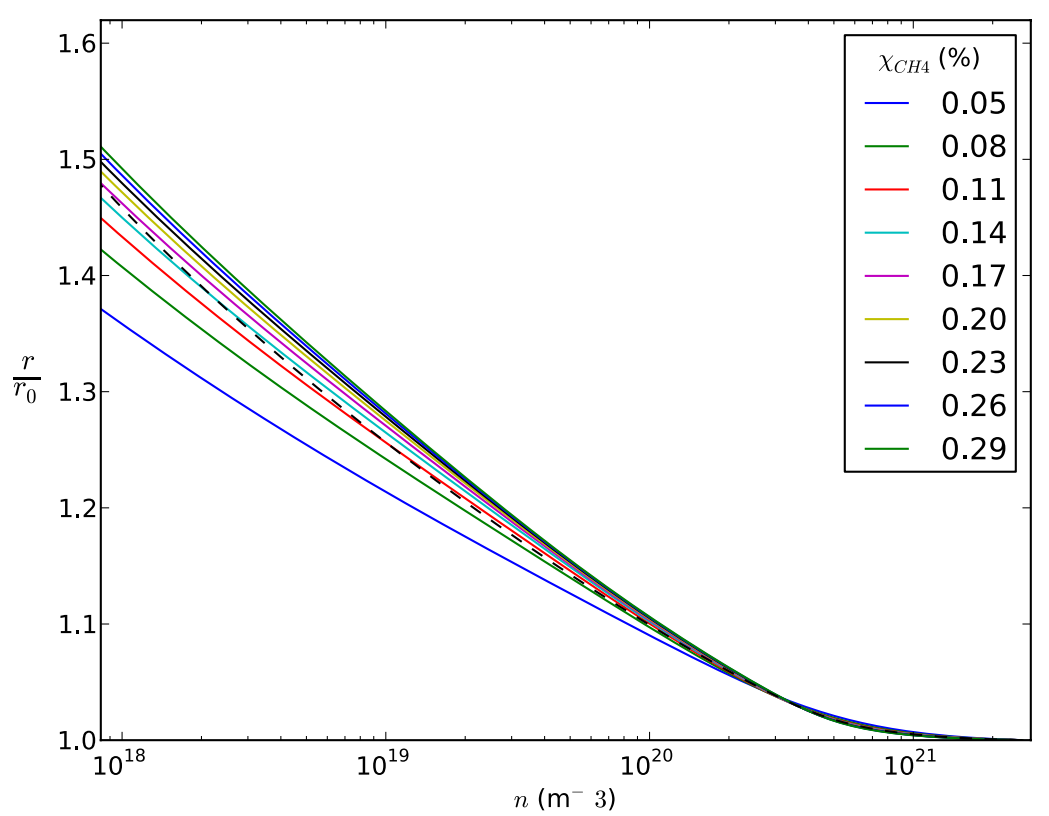

Figure 4-9: Density profiles in the lower atmosphere found by varying the methane mixing ratio. Also plotted as a dotted line is the best-fit solution from Zalucha et al. (2011) to the Siding Spring stellar occultation. The surface pressure was set to $1.32 \mathrm{~Pa}$.

In Figure (4-9), the density in the lower atmosphere can be plotted for several values of methane mixing ratio between $0.05-0.29 \%$. The density from the best-fit solution from Zalucha et al. (2011) to the Siding Spring 2006 stellar occultation is also shown in Figure (4-8). The best-fit solution is bounded by solutions with mixing ratios of $0.08 \%$ and $0.20 \%$, which is below the $0.94 \pm 0.27 \%$ used in Zalucha et al. (2011). Even though I used the same heating model, the $\mathrm{CH}_{4}$ mixing ratio is lower due to the inclusion of adiabatic cooling and small corrections to the code. This range of $\mathrm{CH}_{4}$ mixing ratios is 
below the value of $\chi_{\mathrm{CH}_{4}}=0.6_{-0.3}^{+0.6} \%$ determined from the spectroscopic observations of Lellouch et al. (2011).

In the Zalucha et al (2011) solution, the temperature does not decrease significantly above the peak temperature due to the zero heat flux upper boundary condition and lack of adiabatic cooling. Since I find the temperature does decrease above $r=1.2 r_{0}$, the atmospheric scale heights are different. Therefore, it is of interest to repeat the fitting to the stellar occultation data in order to determine $p_{0}$ and $\chi_{C H 4}$ using my models, since there are several important differences in these solutions from those models of the lower atmosphere used in previous fits to the occultation data.

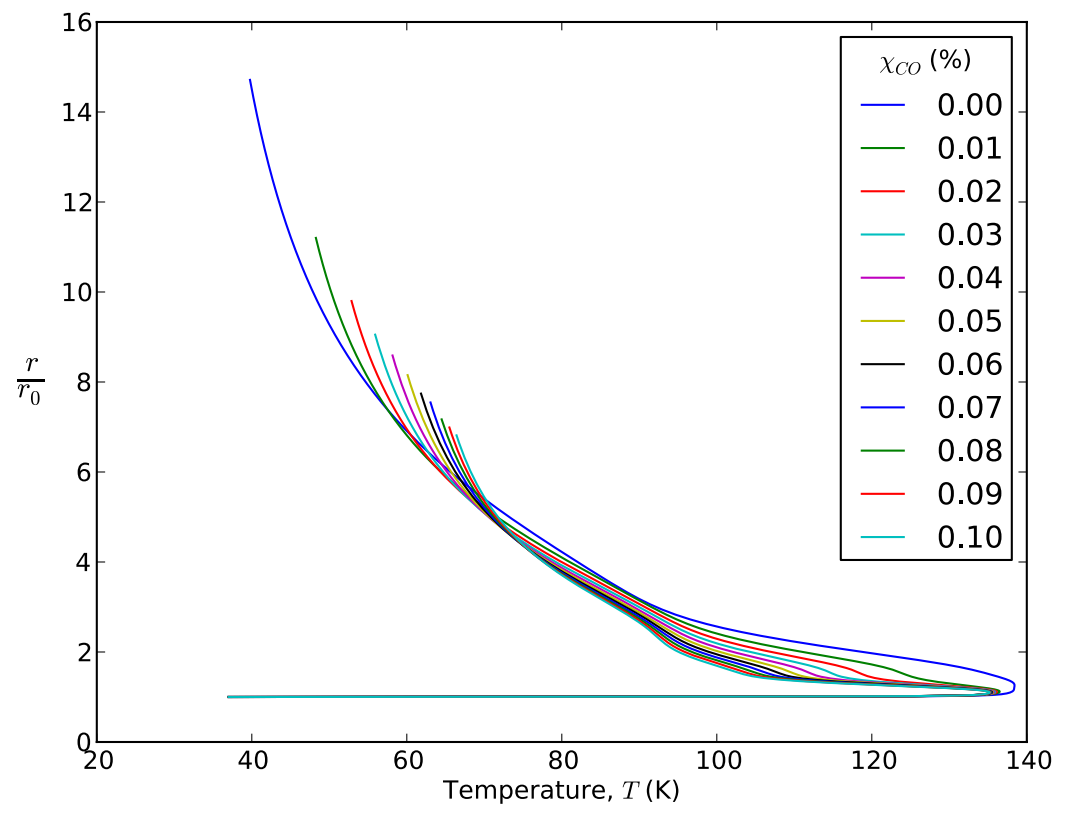

Figure 4-10: Temperature solutions for carbon monoxide mixing ratio between 0.0 and $0.1 \%$.

Zalucha et al. (2011) found the $\mathrm{CO}$ mixing ratio could not be effectively constrained since at those altitudes sensitive to stellar occultations, the $\mathrm{CH}_{4}$ heating and cooling is dominant. Above $r=1.2 r_{0} \mathrm{CO}$ is more efficient at cooling the atmosphere than $\mathrm{CH}_{4}$, therefore the $\mathrm{CO}$ mixing ratio is important to the upper atmosphere. Although, 
since the adiabatic cooling dominates above $r=1.5 r_{p}$ the variability in temperature due to the CO missing ratio is lessened compared to Strobel et al. (1996) and Zalucha et al. (2011). The calculated temperature profiles in Figure (4-9) show that the exobase is sensitive to the $\mathrm{CO}$ mixing ratio. With the atmosphere becoming significantly extended for mixing ratios below the $0.05 \%$ base value. At $30 \mathrm{AU}$ and solar minimum, I find the total escape rate without $\mathrm{CO}$ present to be $8.1 \times 10^{27} \mathrm{~s}^{-1}$ compared to $3.0 \times 10^{27} \mathrm{~s}^{-1}$ with $\chi_{C O}=0.0005$.

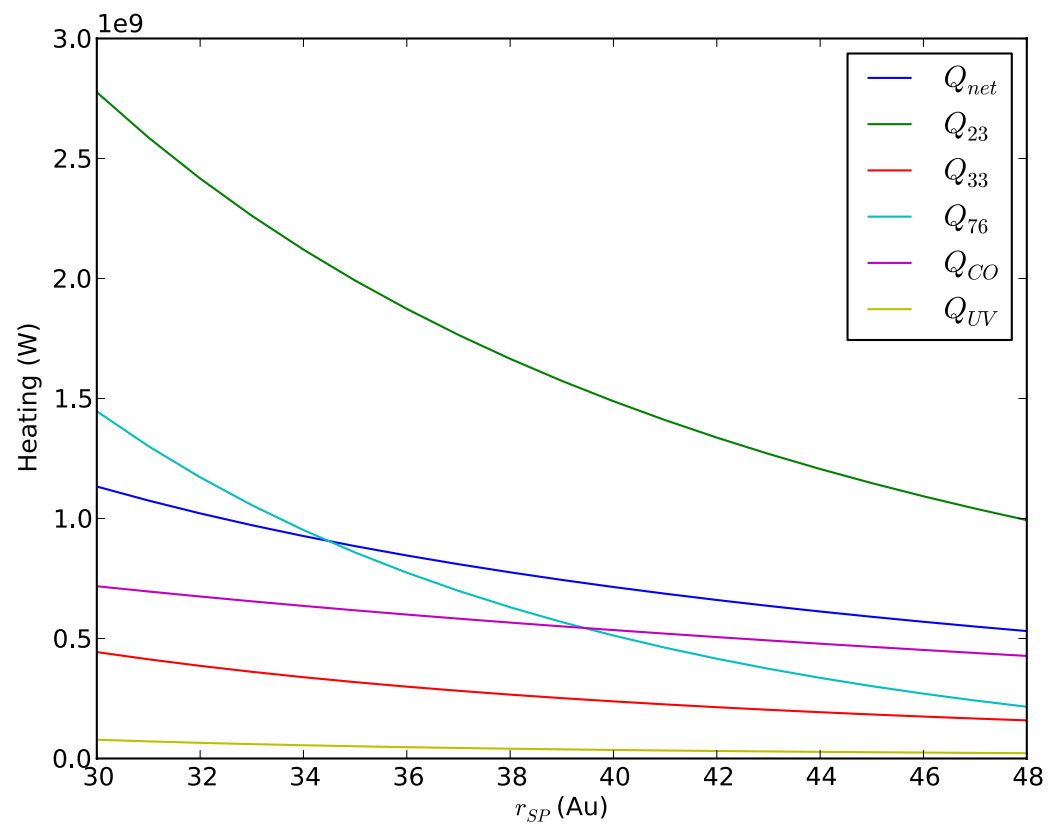

Figure 4-11: Integrated heating rates through Pluto's eccentric orbit. The solar UV activity was fixed to the solar minimum case.

The change in distance from the sun to Pluto, $r_{s p}$ from $\sim 30-48 \mathrm{AU}$, due to Pluto's eccentric orbit has a significant effect on its atmosphere. I found the escape rate varied from $3.1 \times 10^{27} \mathrm{~s}^{-1}$ at perihelion to $0.5 \times 10^{27} \mathrm{~s}^{-1}$ at aphelion and the exobase radius varied from $8.2 r_{0}$ at perihelion to $4.7 r_{0}$ at aphelion. Both the escape rate and exobase radius were found to vary proportional to $r_{s p}^{-2}$. These correlations are expected since the total 
escape rate and exobase radius vary proportional to the integrated UV heating, $Q_{U V}$, which itself should decrease approximately proportional to $r_{s p}^{-2}$. With the surface pressure held fixed, the $\mathrm{N}_{2}$ column density at the surface varies by less than $1 \%$. 


\section{Conclusion}

I compared two methods (fluid-DSMC and fluid-Jeans) for modeling the upper thermosphere and characterizing atmospheric escape. Having shown earlier that such models of atmospheric escape can be scaled (Volkov et al., 2011), I considered Pluto's atmosphere as an example because of the anticipated $\mathrm{NH}$ encounter. I found that, in the presence of significant heating, the fluid-Jeans total escape rate did not change significantly from that obtained from recent hydrodynamic models. However, many other characteristics of the atmosphere are affected, namely the exobase altitude and the existence of a sonic point in the region where the atmosphere is collisional.

The fluid-DSMC model requires no assumptions on the upper boundary conditions, I used this as a baseline for testing fluid models that require assumptions about the particle and energy flux at the upper boundary. By iteration, a consistent fluidDSMC describes the escape rate and energy escape rate as well as the density, temperature and vertical flow speed out to 10 's of planetary radii. The escape rate found is approximately 2 times the Jeans escape rate, but this value changes slowly with the amount of heat deposited in the upper atmosphere.

The fluid-Jeans model uses the Jeans escape for an upper boundary condition as an approximation to the DSMC model in the exobase region. The resulting escape rate of the fluid-Jeans model is surprisingly close to that given by the fluid-DSMC model; the small differences in escape rates do not imply that the temperature and density structures are similar in the two models. By scaling the Jeans escape rate by a factor between 2 and 3 the results become closer to the fluid-DSMC. 
I also showed that the energy-limited escape rate, often used for estimating atmospheric loss from exoplanets, was surprisingly accurate over a broad range of heating conditions from solar minimum to solar maximum. Since the structures of exoplanet atmospheres are usually not known, I also show that in applying this estimate one should use a radius associated with the FUV/EUV absorption radius or, better, use that radius for which the horizontal line of sight optical depth is unity. This radius can be roughly estimated by scaling the visible radius using the ratio of the cross sections, the visible light scattering cross section to the FUV/EUV absorption cross, along with a scale height estimated using the equilibrium temperature. In this way, the results can be applied when modeling the evolution of exoplanet atmospheres.

I adapted the non-LTE IR heating and cooling models for methane and carbon monoxide of Strobel et al. (1996) to create a full atmosphere model of Pluto. By modeling the full atmosphere, I made no assumptions about the state of the middle atmosphere. I showed there is overlap between the IR and UV heating of methane that was not found when the atmosphere was not modeled in its entirety. Adiabatic cooling due to atmospheric escape, usually neglected in the lower atmosphere, has a small effect on the structure of lower atmosphere, but is important to energy budget when the UV heating rate was high. The newly calculated structure in the middle atmosphere can now be used to aid future GCM models of Pluto's atmosphere to correctly determine their boundary conditions. It will also be used to make predictions for the $\mathrm{NH}$ encounter.

This new model makes a number of important predictions. It predicts a smaller mixing ratio for methane, about $0.1 \%$ as opposed to the $1 \%$ required previously, to produce the steep temperature gradient from the surface. It also shows that the escape rate 
is likely not to exceed $10^{28} \mathrm{~s}^{-1}$. Further, I demonstrated the sensitivity of escape rate, exobase altitude, and temperature structure to the surface pressure, methane mixing ratio, carbon monoxide mixing ratio, and the Pluto-Sun distance. With the new model, the surface parameters can be refined by fitting the atmospheric profile to the stellar occultation measurements as was done in Zalucha et al. (2011).

In anticipation of the NH encounter, I simulated Pluto's full atmosphere using the fluid-DSMC model. The atmosphere structure at the encounter can be bounded by the results of the solar minimum and solar mean UV heating cases. The exobase is found between at a radius 8000 and $10000 \mathrm{~km}$, and the escape rate between 2.2 and $3.4 \times 10^{27} \mathrm{~s}^{-1}$. Preliminary results of this model have been presented to the NH science team to aid in the mission planning of the encounter. 


\section{References}

Abramowitz, M. J., Stegun, I. A., 1965. Handbook of Mathematical Functions. Dover. Apruzese, J. P., 1980. The diffusivity factor re-examined. Journal of Quantitive Spectroscopy and Radiative Transfer 24, 461-470.

Bagenal, F., McNutt, R. L., 1989. Pluto's interaction with the solar wind. Geophysical Research Letters.

Bird, G. A., 1963. Approach to translational equilibrium in a rigid sphere gas. Physics of Fluids 6, 1518.

Bird, G. A., 1994. Molecular Gas Dynamics and the Direct Simulation of Gas Flow. Clarendon Press.

Boursier, C., Menard, J., Doyennette, L., Menard-Bourcin, F., 2003. Rovibrational relaxation of methane in $\mathrm{CH}_{4}-\mathrm{N}_{2}$ mixtures: Time-resolved IR-IR double-resonance measurements at $193 \mathrm{k}$ and kinetic modeling. The journal of physical chemistry. A 107 (27), 5280-5290.

Cassidy, T. A., Johnson, R. E., 2010. Collisional spreasing of Enceladus' neutral cloud. Icarus 209, 696-703.

Cercignani, C., 2000. Rarefied Gas Dynamics. Cambridge University Press.

Chassefie`re, E., 1996. Hydrodynamic escape of hydrogen from a hot water-rich atmosphere: The case of Venus. Journal of Geophysical Research. 
Cruikshank, D. P., Roush, T. L., Owen, T. C., Geballe, T. R., de Bergh, C., Schmitt, B., Brown, R. H., Bartholomew, M. J., 1993. Ices on the surface of Triton. Science 261 (5122), 742-745.

Cui, J., Yelle, R. V., Volk, K., 2008. Distribution and escape of molecular hydrogen in Titan's thermopshere and exosphere. Journal of Geophysical Research 113. E. W.

Lemmon, E. W., Jacobsen, R. T., 2003. Viscosity and thermal conductivity equations for nitrogen, oxygen, argon, and air. International Journal of Thermophysics 25 (1), 2169.

Elliot, J. L., Dunham, E. W., Bosh, A. S., Slivan, S. M., Young, L. A., Wasserman, L. H., Millis, R. L., 1989. Pluto's atmosphere. Icarus 77, 148-177.

Erwin, J. T., Tucker, O. J., Johnson, R. E., 2013. Hybrid fluid/kinetic modeling of Pluto's escaping atmosphere. Icarus (in review).

Forster, P. M., Fomichev, V. I., Rozanov, E., Cagnazzo, C., Jonsson, A. I., Langematz, U., Fomin, B., Iacono, M. J., Mayer, B., Mlawer, E., Myhre, G., Portmann, R. W., Akiyoshi, H., Falaleeva, V., Gillett, N., Karpechko, A., Li, J., Lemennais, P., Morgenstern, O., Oberla nnder, S., Sigmond, M., Shibata, K., 2011. Evaluation of radiation scheme performance within chemistry climate models. Journal of Geophysical Research.

Greaves, J. S., Helling, C., Friberg, P., 2011. Discovery of carbon monoxide in the upper atmosphere of Pluto. Monthly Notices of the Royal Astronomical Society: Letters 414, L36-L40.

Gruzinov, A., 2011. The rate of thermal atmospheric escape. ArXiv:1101:1103. 
Guo, Y., Farquhar, R. W., 2005. New Horizons Pluto-Kuiper belt mission: design and simulation of the Pluto-Charon encounter. Acta Astronautica 56 (3), 421 - 429.

Hunten, D. M., Watson, A. J., 1982. Stability of Pluto's atmosphere. Icarus 51, 665-667.

Jeans, J., 1925. The Dynamic Theory of Gases, 4th Edition. Cambridge, London/New York.

Johnson, R. E., 2010. Thermally driven atmospheric escape. The Astrophysical Journal $716(2), 1573$.

Johnson, R. E., Combi, M. R., Fox, J. L., Ip, W. H., Leblanc, F., McGrath, M. A., Shematovich, V. I., Strobel, D. F., Waite, Jr, J. H., 2008. Exospheres and atmospheric escape. Space Science Reviews 139, 355-197.

Johnson, R. E., Volkov, A. N., Erwin, J. T., 2013. Molecular-kinetic simulations of escape from the ex-planet and exoplanets: Criterion for transonic flow. The Astrophysical Journal Letters 768 (1), L4.

Krasnopolsky, V. A., March 1999. Hydrodynamic flow of $\mathrm{N}_{2}$ from Pluto. JGR 104 (E3), $5955-5962$.

Kusnierkiewicz, D. A., Hersman, C. B., Guo, Y., Kubota, S., McDevitt, J., 2005. A description of the Pluto-bound New Horizons spacecraft. Acta Astronautica 57, 135144.

Lammer, H., Odert, P., Leitzinger, M., Khodachenko, M. L., Panchenko, M., Kulikov, Y. N., Zang, T. L., Lichtenegger, H. I. M., Erkaev, N. V., Wuchterl, G., Micela, G., Penz, T., Biernat, H. K., Weingrill, J., Stellar, M., Ottacher, H., Hasiba, J., Hanslmeier, A., October 2009. Determining the mass loss limit for close-in 
exoplanets: what can we learn from transit obervations? Astronomy and Astrophysics $506(1), 399-410$.

Lellouch, E., de Bergh, C., Sicardy, B., Ka üfl, H. U., Smette, A., 2011. High resolution spectroscopy of Pluto's atmosphere: detection of the $2.3 \mu \mathrm{m} \mathrm{CH}_{4}$ bands and evidence for carbon monoxide. Astronomy and Astrophysics 530, L4.

Lellouch, E., Sicardy, B., de Bergh, C., Ka üfl, H. U., Kassi, S., Campargue, A., 2009. Pluto's lower atmosphere structure and methane abundance from high-resolution spectroscopy and stellar occultations. Astronomy and Astrophysics 495, L17-L21.

Lin, R. P., Jakosky, B., 2012. The 2013 Mars Atmosphere and Volatile Evolution (MAVEN) Mission to Mars. In: 39th COSPAR Scientific Assembly. Vol. 39 of COSPAR Meeting. p. 1089.

Liou, K., 2002. An Introduction to Atmospheric Radiation, 2nd Edition. Academic Press, San Diego, California, USA.

McNutt, Jr, R. L., 1989. Models of Pluto's upper atmosphere. Geophysical Research Letters $16(11), 1225-1228$.

Merryfield, W. J., Shizgal, B. D., 1994. Discrete velocity model for an escaping singlecomponent atmosphere. Planetary and Space Science 42 (5), 409 - 419.

Murray-Clay, R. A., Chiang, E. I., Murray, N., Mar. 2009. Atmospheric Escape From Hot Jupiters. The Astrophysical Journal 693, 23-42.

Owen, T. C., Roush, T. L., Cruikshank, D. P., Elliot, J. L., Young, L. A., Bergh, C. d., Schmitt, B., Geballe, T. R., Brown, R. H., Bartholomew, M. J., 1993. Surface ices and the atmospheric composition of Pluto. Science 261 (5122), pp. 745-748. 
Parker, E. N., 1964a. Dynamic properties of stellar coronas and stellar winds: I. integration of the momentum equation. Astrophysics Journal 139, 72.

Parker, E. N., 1964b. Dynamic properties of stellar coronas and stellar winds: II. integration of the heat-flow equation. Astrophysical

Journal 139, 93. Sicardy, B., Widemann, T., Lellouch, E., Veillet, C., Cuillandre, J. C., Colas, F., Roques, F., Beisker, W., Kretlow, M., Lagrange, A. M., Gendron, Lacombe, F., Lecacheux, J., Birnbaum, C., and. Leyrat, A. F., Maury, A., Raynaud, E., Renner, S., Schultheis, M., Brooks, K., Delsanti, A., Hainaut, O. R., Gilmozzi, R., Lidman, C., Spyromilio, J., Rapaport, M., Rosenzweig, P., Naranjo, O., Porras, L., D' ıaz, F., Calderón, H., Carrillo, S., Carvajal, A., Recalde, E., Cavero, L. G., Montalvo, C., Barr'ia, D., Campos, R., Duffard, R., Levato, H., 2003. Large changes in Pluto's atmosphere as revealed by recent stellar occultations. Nature 424, 168-170.

Siddles, R., 1994. The vibrational deactivation of the bending modes of $\mathrm{CD}_{4}$ and $\mathrm{CH}_{4}$ measured down to $90 \mathrm{~K}$. Chemical Physics 188, 99-105.

Strobel, D. F., 2008. N 2 escape rates from Pluto's atmosphere. Icarus 193, 612-619.

Strobel, D. F., 2009. Titan's hydrodynamically escaping atmosphere: Escape rates and the structure of the exobase region. Icaraus 202, 632-641.

Strobel, D. F., Zhu, X., Summers, M. E., Stevens, M. H., 1996. On the thermal structure of Pluto's atmosphere. Icarus 120, 266-289.

Tenishev, V., Combi, M., Davidson, B., 2008. A global kinetic model for cometary comae: the evolution of the coma of the Rosetta target comet ChuryumovGerasimenko throughout the mission. The Astrophysical Journal 685, 659-677. 
Tian, F., Kasting, J. F., li Liu, H., Roble, R. G., 2008. Hydrodynamic planetary thermosphere model: 1. Response of the Earth's thermosphere to extreme solar euv conditions and the significance of adiabatic cooling. Journal of Geophysical Research: Planets 113 (E5).

Tian, F., Kasting, J. F., Solomon, S. C., 2009. Thermal escape of carbon from the early martian atmosphere. Geophysical Research Letters 36, 2205.

Tucker, O., Erwin, J., Deighan, J., Volkov, A., Johnson, R., 2012. Thermally driven atmospheric escape from Pluto's atmosphere: a combined fluid/kinetic model. Icarus $217,408-415$.

Tucker, O. J., Erwin, J. T., Johnson, R. E., Volkov, A. N., Cassidy, T. A., 2010. Fluid/kinetic hybrid simulation of atmospheric escape: Pluto. In: 27th International Symposium on Rarefied Gas Dynamics.

Tucker, O. J., Johnson, R., June 2009. Thermally driven atmospheric escape: Monte Carlo simulations for Titan's atmosphere. Planetary and Space Science 57, 18891894.

Valencia, D., Ikoma, M., Guillot, T., Nettelmann, N., 2010. Composition and fate of short-period super-Earths: The case of CoRoT-7b. Astronomy and Astrophysics.

Volkov, A., Johnson, R., Tucker, O., Erwin, J., 2011a. Thermally driven atmospheric escape: Transition from hydrodynamic to Jeans escape. The Astrophysical Journal Letters 729 (2), L24.

Volkov, A., Tucker, O., Erwin, J., Johnson, R., 2011b. Kinetic simulations of thermal escape from a single component atmosphere. Physics of Fluids 23. 
Walker, A. C., Gratiy, S. L., Goldstein, D. B., Moore, S. H., Varghese, P. L., Trafton, L. M., Levin, D. A., Stewart, B., 2010. A comprehensive numerical simulation of Io’s sublimation driven atmosphere. Icarus 207, 409-432.

Watson, A. J., Donahue, T. M., Walker, J. C., 1981. The dynamics of a rapidly escaping atmosphere: application to the evolution of Earth and Venus. Icarus 48, 150-166.

Yelle, R. V., 2004. Aeronomy of extra-solar giant planets at small orbital distances. Icarus 170.

Yelle, R. V., Lunine, J. I., 1989. Evidence for a molecule heavier than methane in the atmosphere of Pluto. Nature 339, 288-290.

Young, E. F., French, R. G., Young, L. A., Ruhland, C. R., Buie, M. W., Olkin, C. B., Regester, J., Shoemaker, K., G. Blow, J. B., Christie, G., Gault, D., Lade, B., Natusch, T., 2008. Verticle structure in Pluto's atmosphere from the 2006 June 12 stellar occultation. The Astronomical Journal 136, 1757-1769.

Young, L., Sicardy, B., Widemann, T., Brucker, M. J., Buie, M. W., Fraser, B., Van Heerden, H., Howell, R. R., Lonergan, K., Olkin, C. B., Reitsema, H. J., Richter, A., Sepersky, T., Wasserman, L. H., Young, E. F., 2010. Results from the 2010 Feb 14 and July 4 Pluto occultations. In: AAS Division for Planetary Sciences Meeting Abstracts \#42.

Zalucha, A. M., Gulbis, A. A. S., 2012. Comparison of a simple 2-d Pluto general circulation model with stellar occultation light curves and implications for atmospheric circulation. Journal of Geophysical Research: Planets 117 (E5). 
Zalucha, A. M., Gulbis, A. A. S., Zhu, X., Strobel, D. F., Elliot, J. L., 2011. An analysis of Pluto occultation light curves using an atmospheric radiative-conductive model. Icarus $211,804-818$.

Zhu, X., 1992. The correlated-k coefficients calculated by random band models. Journal of Quantitative Spectroscopy and Radiative Transfer 47, 159-170. Zhu, X., Strobel, D. F., 1990. On the role of vibration-vibration transitions in radiative cooling of the co2 $15 \mathrm{~m}$ band around the mesopause. Journal of Geophysical Research: Atmospheres 95 (D4), 3571-3577. 


\title{
THERMALLY DRIVEN ATMOSPHERIC ESCAPE: TRANSITION FROM HYDRODYNAMIC TO JEANS ESCAPE
}

\author{
Alexey N. Volkov ${ }^{1}$, Robert E. Johnson ${ }^{1,2}$, Orenthal J. Tucker ${ }^{1}$, And Justin T. ERwin ${ }^{1}$ \\ ${ }^{1}$ Materials Science and Engineering Department, University of Virginia, Charlottesville, VA 22904-4745, USA \\ ${ }^{2}$ Physics Department, New York University, NY 10003-6621, USA \\ Received 2010 October 1; accepted 2011 January 25; published 2011 February 16
}

\begin{abstract}
Thermally driven escape from planetary atmospheres changes in nature from an organized outflow (hydrodynamic escape) to escape on a molecule-by-molecule basis (Jeans escape) with increasing Jeans parameter, $\lambda$, the ratio of the gravitational to thermal energy of the atmospheric molecules. This change is described here for the first time using the direct simulation Monte Carlo method. When heating is predominantly below the lower boundary of the simulation region, $R_{0}$, and well below the exobase of a single-component atmosphere, the nature of the escape process changes over a surprisingly narrow range of Jeans parameters, $\lambda_{0}$, evaluated at $R_{0}$. For an atomic gas, the transition occurs over $\lambda_{0} \sim 2-3$, where the lower bound, $\lambda_{0} \sim 2.1$, corresponds to the upper limit for isentropic, supersonic outflow. For $\lambda_{0}>3$ escape occurs on a molecule-by-molecule basis and we show that, contrary to earlier suggestions, for $\lambda_{0}>\sim 6$ the escape rate does not deviate significantly from the familiar Jeans rate. In a gas composed of diatomic molecules, the transition shifts to $\lambda_{0} \sim 2.4-3.6$ and at $\lambda_{0}>\sim 4$ the escape rate increases a few tens of percent over that for the monatomic gas. Scaling by the Jeans parameter and the Knudsen number, these results can be applied to thermally induced escape of the major species from solar and extrasolar planets.
\end{abstract}

Key words: hydrodynamics - planets and satellites: atmospheres - planets and satellites: individual (Pluto, Titan)

\section{INTRODUCTION}

Our understanding of atmospheric evolution is being enormously enhanced by extensive spacecraft and telescopic data on outer solar system bodies and exoplanets. The large amount of data on Titan's atmosphere from the Cassini spacecraft led to estimates of the atmospheric escape rate that, quite surprisingly, differed enormously (see Johnson 2009; Johnson et al. 2009). This was due to a lack of a kinetic model for how escape changes in character from evaporation on a molecule-by-molecule basis, referred to as Jeans escape, to an organized outflow, referred to as hydrodynamic escape, a process of particular interest to exoplanets (e.g., Yelle 2004; Murray-Clay et al. 2009) and to the early stages of atmospheric evolution (e.g., Watson et al. 1981; Hunten 1982; Tian et al. 2008).

Whether escape from a planet's atmosphere is hydrodynamic or Jeans-like is often characterized by the Jeans parameter, $\lambda=$ $|U(r)| /(k T(r))$, where $U(r)$ is a molecule's gravitational energy at distance $r$ from the planet's center, $T$ is the temperature, and $k$ is the Boltzmann constant (e.g., Chamberlain \& Hunten 1987; Johnson et al. 2008). In Hunten (1982), it was suggested that if $\lambda$ decreased to $\sim 2$ above the exobase, then the escape rate is not too different from the Jeans rate, but if $\lambda$ became $\sim 2$ below the exobase, hydrodynamic escape would occur. Because the change in the nature of the escape process was assumed to occur over a broad range of $\lambda$, an intermediate model, the slow hydrodynamic escape (SHE) model, was adopted to describe the outflow from atmospheres such as Pluto's with exobase values $\lambda \sim 10$ (Hunten \& Watson 1982; McNutt 1989; Krasnopolsky 1999; Tian \& Toon 2005; Strobel 2008a). In the SHE model, based on Parker's (1964a, 1964b) model for the solar wind, the fluid equations, accounting for heat conduction, are solved to an altitude above which asymptotic conditions on the temperature and density are applied (e.g., Strobel 2008a). Other recent models couple the Jeans rate (Chassefiere 1996; Tian et al. 2008) or a modified Jeans rate (Yelle 2004; Tian 2009) to a continuum model. Although such approaches can give reasonable approximations, none can overcome a principal drawback of fluid models: the application of near-equilibrium models to a part of the atmosphere, where the gas is rarefied and non-equilibrium effects are essential.

This Letter is aimed at revealing when the kinetic effects in atmospheric escape are important which we examine over a large range of $\lambda$ including both hydrodynamic and Jeans escape. Such effects emerge due to the lack of translational equilibrium in an expanding gas and cannot be quantitatively predicted by familiar fluid models. To make clear when this is important for escape of the primary species, we use a kinetic model for a single-component atmosphere composed of a monatomic or diatomic gas.

\section{DSMC SIMULATIONS}

The transition from hydrodynamic to Jeans escape from planetary bodies is modeled here by the direct simulation Monte Carlo (DSMC) method (e.g., Bird 1994). It is a stochastic method for the numerical solution of flow based on the Boltzmann equation (e.g., Chapman \& Cowling 1970). As in Parker's model, a spherically symmetric, single-component atmosphere is supplied by outgassing from a surface at radius $R_{0}$. This can be the planet's actual surface with a vapor pressure determined by the solar insolation or a radial position in the atmosphere above which little heat is deposited and at which the density and temperature are known. In such a model, escape is driven by thermal conduction and heat flow from below $R_{0}$. In the DSMC method, the gas is simulated by a large number of representative molecules of mass $m$. These molecules are subject to binary collisions and their trajectories are calculated in a gravity field: $U(r)=-G M m / r$, where $M$ is the planet's mass and $G$ is the gravitational constant. The mass of the gas above $R_{0}$ is assumed to be much smaller than $M$ so that self-gravity is neglected.

The velocity distribution at $r=R_{0}$ is maintained to be Maxwellian for molecules with positive velocity component parallel to the local radial direction, $v_{\|}>0$, at a fixed number density, $n_{0}$, temperature, $T_{0}$, and zero gas velocity. The exit 
boundary at $r=R_{1}$ is placed far enough from $R_{0}$ so the flow above $R_{1}$ is approximately collisionless. A molecule crossing $R_{1}$ with $v_{\|}$and $v_{\perp}$ will escape if velocity $v>\left(-2 U\left(R_{1}\right) / m\right)^{1 / 2}$, where $v=\sqrt{v_{\|}^{2}+v_{\perp}^{2}}$, while a molecule with a smaller $v$ will return to $R_{1}$ with $-v_{\|}$and $v_{\perp} . R_{1}$ is chosen to be sufficiently large to not affect the region described.

Although the principal results presented are for hard sphere (HS) collisions, comparisons made earlier using other models gave similar results (Tucker \& Johnson 2009). It is readily shown that for a velocity-independent cross section, the Boltzmann equations, and, hence, the results presented here, can be scaled by two parameters: the source values of the Jeans parameter, $\lambda_{0}$, and a Knudsen number, $K n_{0}=l_{0} / R_{0}$, where $l_{0}$ is the equilibrium mean free path of molecules at $r=R_{0}$. For HS collisions, $l_{0}=\left(2^{1 / 2} n_{0} \sigma\right)^{-1}$, with $\sigma$ being the collision cross section. The Knudsen number often discussed for a planet's atmosphere is $K n(r)=l(r) / H(r)$, where $l(r)$ and $H(r)$ are the local mean free path and the atmospheric scale height, respectively. Since $K n\left(R_{0}\right)=\lambda_{0} K n_{0}$, it can be used instead of $K n_{0}$. The simulations were carried out by fixing $m, \sigma, T_{0}$, and $R_{0}$ with $\lambda_{0}$ and $K n_{0}$ varied by changing $M$ and $n_{0}$. Since the results scale with $\lambda_{0}$ and $K n_{0}$, any two flows with different $m, \sigma, T_{0}, R_{0}, M$, and $n_{0}$ represent the same flow in dimensionless form if $\lambda_{0}$ and $K n_{0}$ are the same. For comparison, we also model atmospheres composed of diatomic molecules with the collisions described by the variable hard sphere (VHS) model combined with the Larsen-Borgnakke (LB; e.g., Bird 1994) model, accounting for energy transfer between translational and internal degrees of freedom. A Maxwell-Boltzmann distribution of the energy of the internal degrees of freedom (Bird 1994) is assumed at $r=R_{0}$ for molecules with $v_{\|}>0$. To represent low-temperature $\mathrm{N}_{2}$ atmospheres, such at those at Pluto, Titan, and Triton, in the VHSLB model the viscosity index is equal to 1 ( $\sigma=\sigma_{\text {ref }} C_{r} / C_{r \text {, ref }}$, where $C_{r}$ is the relative velocity of colliding molecules) and the number of internal degrees of freedom is 2 (Strobel 2008a, 2008b), and $l_{0}=\sqrt{8 k T_{0} /(\pi m)} /\left(n_{0} \sigma_{\text {ref }} C_{r, \text { ref }}\right)$ (Bird 1994).

Simulations are initiated by "evaporation" from the cell at $R_{0}$ until steady flow is reached at large $t$. Steady state is assumed to occur when the number flux $4 \pi r^{2} n(r) u(r)=\Phi$ varies by less than $\sim 1 \%$ across the domain, where $u(r)$ is the gas velocity and $\Phi$ is the escape rate. The independence of $\Phi$ on the simulation time was shown in test simulations, where the time before sampling the molecular parameters was sequentially increased by a factor of two, until no changes in $\Phi$ were found within accepted errors. Jeans escape, $\Phi_{\text {Jeans }}$, is defined by the upward flux of molecules with velocities $v \geqslant(-2 U(r) / m)^{1 / 2}$ at the nominal exobase, $r_{\text {exo }}$, determined by the scale height $\left[l\left(r_{\text {exo }}\right)=H\left(r_{\text {exo }}\right)\right]$ at large $\lambda_{0}$ or by the curvature $\left[l\left(r_{\text {exo }}\right)=r_{\text {exo }}\right]$ at small $\lambda_{0}$. Due to the limited number of simulation particles, accurately representing the tail of the velocity distribution is problematic at large $\lambda_{0}$. In the solar system, $\lambda_{0}$ varies from $\sim 0.01$ for comets to $\sim 10$ for Pluto, and $\sim 40$ for Titan. For the test particle numbers used here, accurate escape rates are obtained for $\lambda_{0}<15$ which allows us to explore the transition region. For accurate escape rates, $R_{1} / R_{0}=40$ was required for $\lambda_{0} \leqslant 10$; for $\lambda_{0}>10, R_{1} / R_{0}=6$ was sufficient. Gas parameters in Figure 1, which can be more sensitive to $R_{1}$, are obtained using $R_{1} / R_{0}=100$.

\section{RESULTS FOR THERMAL ESCAPE}

A study was first carried out for HS collisions for $\lambda_{0}=0$ to 15 and a large range of $K n_{0}$. We focus here on small $K n_{0}$, so that the lower boundary is well into the collision-dominated region of a planet's atmosphere where the fluid equations apply. Atmospheric properties versus $r$ for a number of $\lambda_{0}$ with $K n_{0}=$ 0.001 are shown in Figure 1. The dependence on $r$ of the density, Mach number, and temperature are seen to be similar for small $\lambda_{0}$ $(0,1,2)$, but, quite surprisingly, the radial dependence changes dramatically as $\lambda_{0}$ is increased $(3,10)$ with a distinct transition between 2 and 3 . In particular, the number density $n / n_{0}$ and the ratio $T_{\perp} / T_{\|}$of perpendicular $T_{\perp}$ and parallel $T_{\|}$temperatures at fixed $r / R_{0}$ tend to increase with increasing $\lambda_{0}$ for $\lambda_{0} \leqslant 2$ and tend to decrease with increasing $\lambda_{0}$ for $\lambda_{0} \geqslant 3$. The ratio, $T_{\perp} / T_{\|}$, considered a measure of translational non-equilibrium (Cattolica et al. 1974), remains close to unity in the transition region up to large $r$ (e.g., up to $r / R_{0}=20$ for $\lambda_{0}=3$ ). Using the criteria in Cattolica et al. (1974), the onset of translational non-equilibrium for $\lambda_{0} \leqslant 2$ is characterized by $l(r) / r \sim 0.05$. For $\lambda_{0} \geqslant 3$ at $K n_{0}=10^{-3}$, the transition to non-equilibrium is better characterized in terms of $K n(r)$ with the onset of nonequilibrium increasing with increasing $\lambda_{0}$, e.g., $K n(r) \sim 0.1$ at $\lambda_{0}=3$ and $K n(r) \sim 0.3$ at $\lambda_{0}=10$. Although $T_{\perp}$ decays to zero, as expected at large $r$, it is seen in Figure 1(c) that $T_{\|}$does not go to zero.

More important to escape, the flow in Figure 1(b) is seen to be hypersonic for the small values of $\lambda_{0}$, but, contrary to what has been assumed, it never becomes hypersonic for the larger $\lambda_{0}$ shown, even at very large $r$ well above the exobase. At $\lambda_{0}=3$ the flow is seen to be truly subsonic. At $\lambda_{0}=10$, the flow velocity $u$ does not exceed the isentropic sound speed $\sqrt{(5 / 3) k T / m}$ (here $\left.T=\left(T_{\|}+2 T_{\perp}\right) / 3\right)$. However, $u$ can slightly exceed the isothermal sound speed, $\sqrt{k T / m}$, the critical velocity in Parker's (1964a) model. For example, for $\lambda_{0}=10$ and $K n_{0}=10^{-2}$, $u=\sqrt{k T / m}$ at $r / R_{0} \approx 80$. At $K n_{0}=10^{-3}$ this does not occur until $r / R_{0}$ exceeds 100 . At such distances, $l(r) / H(r)>10^{3}$ so the flow is close to free molecular flow. If $K n_{0}$ is increased, the flow approaches the free molecular limit and can become supersonic very far from the source at arbitrary $\lambda_{0}$. But such flows cannot be quantitatively described using fluid models.

The properties at small $K n_{0}$ for $\lambda_{0} \leqslant 2$ roughly agree with those for an isentropic expansion above its sonic surface at $r=$ $r_{*}$, where $u=\sqrt{(5 / 3) k T / m}$ :

$$
\begin{aligned}
r^{2} n u & =r_{*}^{2} n_{*} u_{*} ; \frac{T}{n^{2 / 3}}=\frac{T_{*}}{n_{*}^{2 / 3}} ; \frac{5}{2} k T+\frac{m u^{2}}{2}-\frac{G M m}{r} \\
& =\frac{5}{2} k T_{*}+\frac{m u_{*}^{2}}{2}-\frac{G M m}{r_{*}} .
\end{aligned}
$$

In Equations (1), $n_{*}, u_{*}$, and $T_{*}$ are evaluated at $r=r_{*}$. For small $\lambda_{0}$ and $K n_{0}, r_{*}$ is located close to $R_{0}$ and hydrodynamic outflow occurs. As $\lambda_{0}$ increases to $\sim 2$, the flow gradually decelerates (Figure 1(b)) and the thickness of the Knudsen layer decreases with decreasing $K n_{0}: r_{*} \rightarrow R_{0}$ (Figure 1(b)), and $T_{*} \rightarrow$ $\sim 0.64 T_{0}$ (Figure $1(\mathrm{c})$ ). The latter is consistent with kinetic simulations of spherical expansion at zero gravity (e.g., Sone \& Sugimoto 1993). Since $(5 / 2) k T_{*}+m u_{*}^{2} / 2-G M m / r_{*} \rightarrow 0$ as $\lambda_{0} \rightarrow \sim 2.1$ a rapid deceleration of the flow occurs. Therefore, for the larger $\lambda_{0}, n / n_{0}$ and $T_{||} / T_{0}$ rise up to $\sim 1$ at $R_{0}$, while the local Mach number rapidly drops below 0.1 . At $\lambda_{0}=2-3$ and small $K n_{0}$, the rapid transition from hydrodynamic outflow to a nearly isothermal atmosphere below the exobase is such that above $\lambda_{0} \sim 2.1$ the sonic point is not reached even at very large $r$ and the atmosphere is gravitationally dominated. Therefore, the transition region is not broad at small $K n_{0}$ and $\lambda_{0} \sim 10$ is well above the region where escape by outflow occurs. This is unlike the prediction of Parker's (1964a, 1964b) model reformulated 

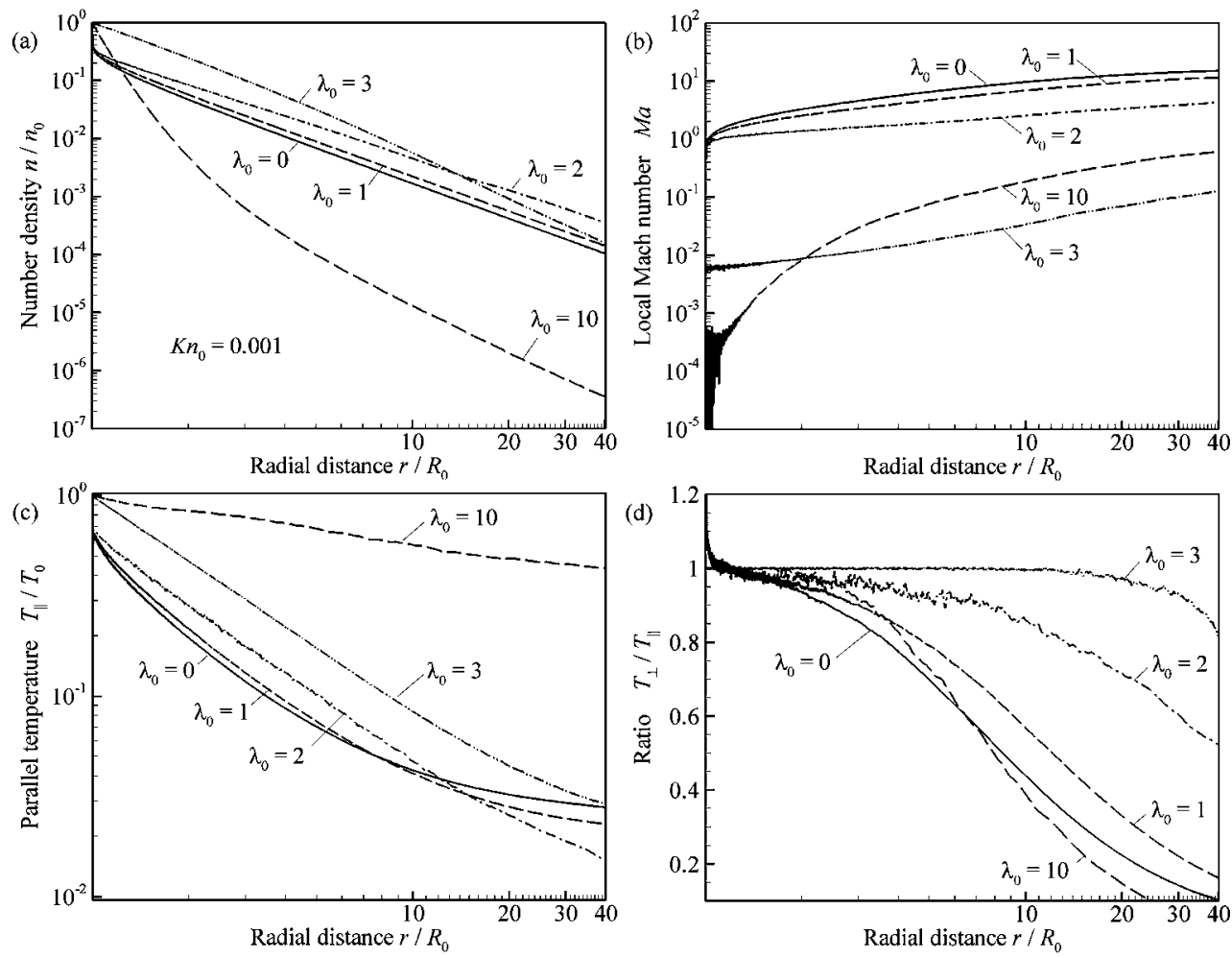

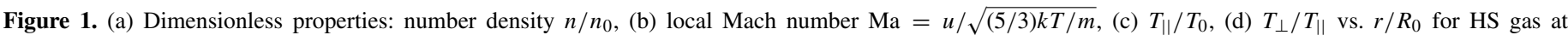

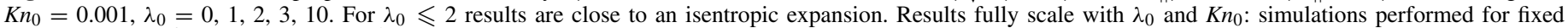
$m=4.65 \times 10^{-26} \mathrm{~kg}, \sigma=7.1 \times 10^{-15} \mathrm{~cm}^{2}$ (cross section for $\mathrm{N}_{2}$ at $90 \mathrm{~K}$; Bird 1994), $T_{0}=100 \mathrm{~K}, R_{0}=1000 \mathrm{~km}$, while $M$ and $n_{0}$ were varied.

for an atomic gas, in which there is a critical point, beyond which supersonic expansion can occur, for every $\lambda_{0}$.

Figure 2 shows the change in the parallel velocity distribution at $r / R_{0}=10$ for planets having different $\lambda_{0}$. For $\lambda_{0} \leqslant 2$, the distribution is shifted toward large $v_{\|}$and $u$ is close to the most probable molecular velocity. For $\lambda_{0} \geqslant 3$, the most probable $v_{\|}$ is seen to be close to zero and escape is provided by the upward moving, high-speed molecules. This fact together with $T_{\perp} \neq T_{\|}$ highlights the non-equilibrium nature of the flow. In addition, for $\lambda_{0} \geqslant 3$ the flow velocity and the molecular escape rate, $\Phi$, cannot be associated with the maximum in the velocity distribution.

The effect of the change in the nature of the flow on $\Phi$ is shown in Figure 3. For the HS model, the ratio of $\Phi$ to the "evaporation" rate at $R_{0}, \Phi_{0,0}=4 \pi R_{0}^{2} n_{0} \sqrt{k T_{0} /(2 \pi m)}$ drops dramatically between $\lambda_{0}=2$ and 3 for both $K n_{0}=0.001$ and 0.0003 . Up to $\lambda_{0}=2$, the ratio is very close to $\sim 0.82$, the rate found in the absence of gravity as in a comet-like expansion (e.g., Cong \& Bird 1978; Crifo et al. 2002; Tenishev et al. 2008). The $\sim 0.82$ is due to collisions in the Knudsen layer causing the return flow. As the atmospheric outflow is choked off by the increased gravitational binding, $\Phi / \Phi_{0,0}$ rapidly drops a couple of orders of magnitude between $\lambda_{0}=2$ and 3 . In this region, escape is a product of a rapidly decreasing fraction of the velocity distribution with $v>v_{\text {esc }}$ (Figure 2) and a rapidly decreasing flow velocity (Figure $1(\mathrm{~b})$ ). Therefore, for $\lambda_{0} \geqslant 6$, it is seen that the escape rate is not very different from the Jeans rate contrary to what had been suggested. The ratio of $\Phi$ to the Jeans rate varies from 1.7 to 1.4 as $\lambda_{0}$ goes from 6 to 15 , consistent with Tucker \& Johnson (2009). A modified Jeans rate, accounting for the nonzero $u$, can provide an approximation to $\Phi$ at $\lambda_{0} \leqslant 6$ (Volkov et al. 2011). At $\lambda_{0} \geqslant 3$, values of $\Phi / \Phi_{0,0}$ even at $K n_{0} \sim 10^{-4}$ do not converge yet to values of the escape rate characteristic for the continuum limit, $K n_{0} \rightarrow 0$ (Figure 4).

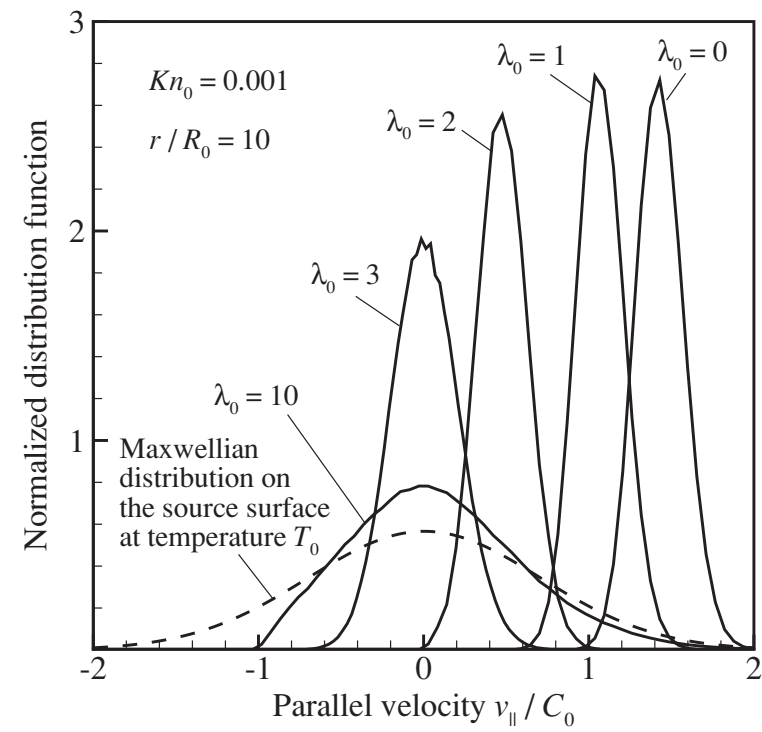

Figure 2. Normalized distributions of parallel velocity $v_{\|} / C_{0}$ in HS gas for $\lambda_{0}=0,1,2,3,10$ at $K n_{0}=0.001$ and $r / R_{0}=10$. Black dashed curve: Maxwellian distribution at $R_{0} . C_{0}=\sqrt{2 k T_{0} / m}$.

For comparison, the escape rate for an $\mathrm{N}_{2}$ atmosphere calculated using the VHS-LB model is also shown in Figure 3 for $K n_{0}=0.001$. The transition region remains narrow, shifting to $\lambda_{0} \sim 2.4-3.6$, but the lower limit, $\lambda_{0} \sim 2.4$, still approximately corresponds to the upper limit for isentropic outflow for an $\mathrm{N}_{2}$ atmosphere. For $\lambda_{0} \sim 6-15$, the escape rate for $\mathrm{N}_{2}$ is $\sim 50 \%-15 \%$ larger than that for the HS gas due to the rotational-translational energy exchange. The rate is still only $\sim 2.4-1.4$ times the Jeans rate at the exobase for $\lambda_{0} \sim 6-15$, and not orders of magnitude 


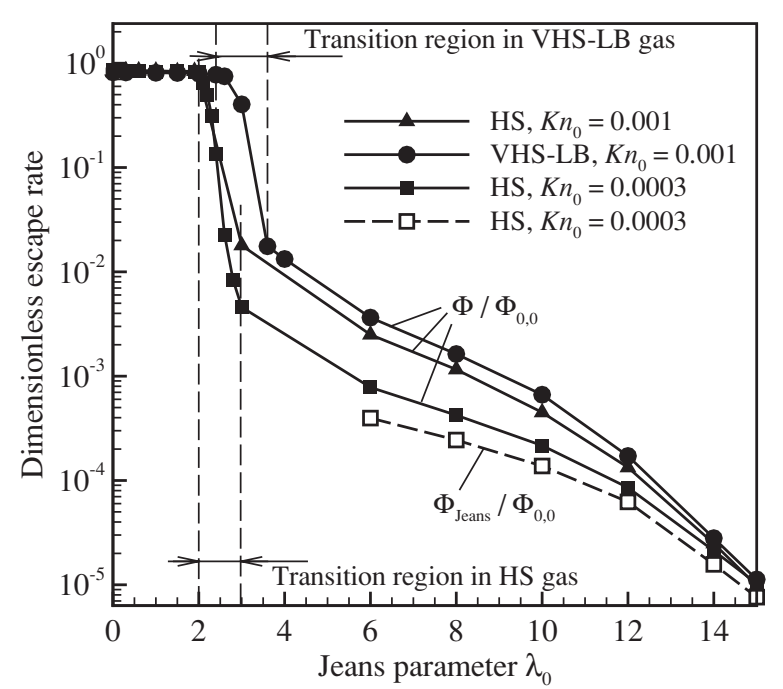

Figure 3. Dimensionless escape rate $\Phi / \Phi_{0,0}$ (solid curves) and Jeans escape rate $\Phi_{\text {Jeans }} / \Phi_{0,0}$ evaluated at the nominal exobase (dashed curve) vs. $\lambda_{0}$ at $K n_{0}=0.001$ (circles and triangles) and $K n_{0}=0.0003$ (squares). Square and triangles are for a gas composed of HS molecules; circle symbols: for a diatomic gas composed of VHS molecules with viscosity index equal to 1 and two internal degrees of freedom, described by the Larsen-Borgnakke (LB) model. $\left.\Phi_{0,0}=4 \pi R_{0}^{2} n_{0} \sqrt{k T_{0} /(2 \pi m}\right)$ is the evaporation rate on $R_{0}$. Vertical lines indicate transition regions.

larger as predicted for $\mathrm{N}_{2}$ at Pluto (Hunten \& Watson 1982; McNutt 1989; Krasnopolsky 1999; Strobel 2008a) and Titan (Strobel 2008b).

In order to further test the fluid models, the heat flux calculated based on its kinetic definition (Bird 1994) is compared in Figure 5 to that in the fluid model for $\lambda_{0}=10$, a value relevant to Pluto and considered intermediate between cometary outflow and terrestrial atmospheres. It has been argued that at such $\lambda_{0}$ escape driven by thermal conduction can be continued into the exobase region (e.g., Strobel 2008b) a viewpoint criticized (Johnson 2010). It is seen that even a couple of scale heights below the exobase the heat flux is not well described by the Fourier law, $-\kappa(T) d T / d r$, where $\kappa(T)$ is the thermal conductivity for an HS gas (Chapman \& Cowling 1970; Bird 1994). This law drastically overestimates the energy flux, consistent with $\lambda_{0}=10$ being well above the transition region. Thus, fluid models should be applied well below the exobase where the effects of translational non-equilibrium are negligible.

Our results can be used to evaluate calculations of loss rates for the principal atmospheric species. Although $\mathrm{H}_{2}$ escape from Titan is significant, the large thermally induced loss rate estimated for the principal species is clearly incorrect, but plasma-induced escape can be important (Westlake et al. 2011) as predicted (Johnson et al. 2009). Simulations for trace species such as $\mathrm{H}_{2}$ at Titan are in progress. For Pluto's predominantly nitrogen atmosphere, when all of the heating is assumed to be below $R_{0}=1450 \mathrm{~km}$, the "zero heating case" $(Q=0)$ in Strobel (2008a) corresponds to $\lambda_{0} \sim 23$. Therefore, the escape rate is close to the Jeans rate, giving a loss rate is orders of magnitude below that estimated $\left(\sim 5 \times 10^{28} \mathrm{amu} \mathrm{s}^{-1}\right)$. Of more interest is the loss rate for solar medium heating in Strobel (2008a). $K n_{0}=0.01$ occurs at $R_{0} \sim 3600 \mathrm{~km}$, which is well above the solar heating maximum and corresponds to $\lambda_{0} \sim 10$. Using the results in Figure 4, the loss rate would be $\sim 7 \times 10^{27}$ amu s${ }^{-1}$, indicating that the energy limited value predicted $(\sim 9 \times$ $10^{28} \mathrm{amu} \mathrm{s}^{-1}$ ) is inconsistent with the calculated atmospheric structure. Therefore, it is clear that modeling in support of the

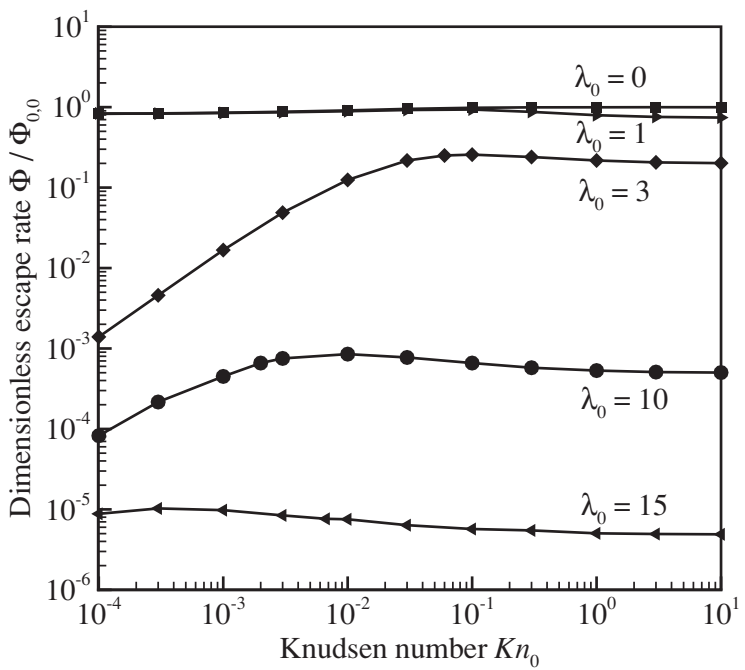

Figure 4. Dimensionless escape rate $\Phi / \Phi_{0,0}$ vs. $K n_{0}$ at $\lambda_{0}=0,1,2,3,10$ for HS molecules.

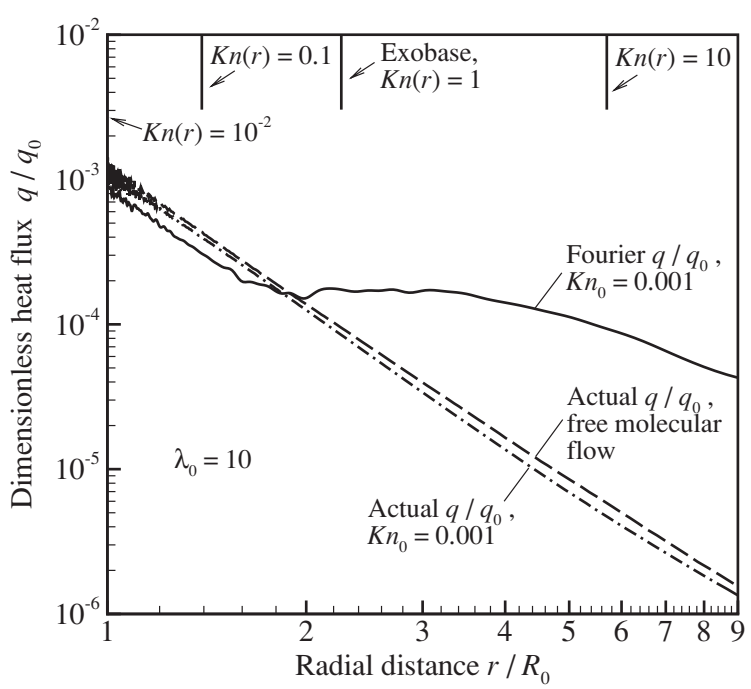

Figure 5. Dimensionless heat flux $q / q_{0}$ vs. $r / R_{0}$ for $\lambda_{0}=10$ in HS gas: $K n_{0} \rightarrow \infty$ (free molecular flow: dashed curve); $K n_{0}=0.001$ (dash-dotted curve). Solid curve: dimensionless Fourier heat flux, $q=-\kappa(T) d T / d r$ calculated from $T(r)$ found in DSMC simulations at $K n_{0}=0.001$ with thermal conductivity $\kappa(T)$ given by the first approximation of Chapman-Enskog for an HS gas (Chapman \& Cowling 1970) and $q_{0}=n_{0} k T_{0} \sqrt{2 k T_{0} / m}$. Upper axis: $K n(r)=l(r) / H(r)$.

New Horizon mission to Pluto will require a kinetic description of escape. Since the lower boundary occurs at a very small Knudsen number, a kinetic model of escape can be iteratively coupled to a fluid description of the lower atmosphere (Tucker et al. 2011). In a study of the EUV heating of Earth's early atmosphere, Tian et al. (2008) found the onset of hydrodynamic escape of oxygen, and the resulting adiabatic cooling of the thermosphere, occurs at an exobase having a value of $\lambda \sim 5.3$. Since this is well above the heating peak and corresponds to a $K n_{0} \sim 0.2$, the inferred onset is in disagreement with the results presented here. As they used a modified Jeans rate, their escape rate is only a factor of $\sim 4$ too big. What is more important is the difference in the atmospheric structure near the exobase, which is dominated by non-equilibrium effects. Therefore, the fluid calculations of atmospheric escape from solar system bodies and exoplanets should be tested against kinetic simulations. 


\section{SUMMARY}

A kinetic model was used to study the change in the nature of atmospheric escape from hydrodynamic to escape on a molecule-by-molecule basis. When heat is deposited primarily below the lower boundary of the simulation region, $R_{0}$, and the collision cross sections are energy-independent, the results presented here can be scaled by two parameters evaluated at $R_{0}$ : The Jeans parameter, $\lambda_{0}$, and the Knudsen number, $K n_{0}$ (or $K n\left(R_{0}\right)$ ). For $R_{0}$ in the collision dominated regime (small $\left.K n_{0}\right)$ the transition from hydrodynamic to Jeans-like escape is found to occur over a surprisingly narrow range of $\lambda_{0}$. Recently, Gruzinov (2011) showed that for $K n_{0} \rightarrow 0$ a hydrodynamic model of thermal escape, with appropriate boundary conditions, can give a sharp transition in escape rate. That is, below a critical value of $\lambda_{0} \quad(\sim 2.1$ and $\sim 2.4$ for monatomic and diatomic gases), hydrodynamic outflow occurs and is roughly described by an isentropic expansion starting from the sonic surface. Above the transition regime (at $\lambda_{0}>\sim 3$ and $\lambda_{0}>$ $\sim 3.6$ for monatomic and diatomic gases), escape occurs on a molecule-by-molecule basis. Depending on $\lambda_{0}$, the fluid approximation for a monatomic gas breaks down when $K n(r)<$ $0.1-0.3$ for $\lambda_{0}=3-10$, which is well below the exobase as seen in Figure 5. However, when a trace species is present, such as $\mathrm{H}_{2}$ at Titan, the breakdown occurs at smaller $K n(r)$.

For $\lambda_{0}>\sim 6$ we show that the escape rates do not deviate enormously from the Jeans rate and we find only moderate differences between escape rates from monatomic and diatomic atmospheres. These results differ from what was predicted by Strobel (2008a, 2008b, 2009) based on a Parker-type (Parker 1964a, 1964b) model for a nitrogen atmosphere. Although our kinetic simulations are for a single-component, spherically symmetric atmosphere, the results have implications for complex atmospheres. Therefore, re-evaluation of studies for exoplanets, early terrestrial atmospheres, and the atmosphere of Pluto, soon to be visited by New Horizon, should be carried out using kinetic models of the upper atmosphere.

This work is supported by NASA's Planetary Atmospheres Program and the Cassini Data Analysis Program.

\section{REFERENCES}

Bird, G. A. 1994, Molecular Gas Dynamics and the Direct Simulation of Gas Flows (Oxford: Clarendon), 218

Cattolica, R., Robben, F., Talbot, L., \& Willis, D. R. 1974, Phys. Fluids, 17, 1793

Chamberlain, J. W., \& Hunten, D. 1987, Theory of Planetary Atmosphere (New York: Academic)

Chapman, S., \& Cowling, T. G. 1970, The Mathematical Theory of Nonuniform Gases (3rd ed.; Cambridge: Cambridge Univ. Press)

Chassefiere, E. 1996, Icarus, 124, 537

Cong, T. T., \& Bird, G. A. 1978, Phys. Fluids, 21, 327

Crifo, J. F., Loukianov, G. A., Rodionov, A. V., Khanlarov, G. O., \& Zakharov, V. V. 2002, Icarus, 156, 249

Gruzinov, A. 2011, arXiv:1101.1103

Hunten, D. M. 1982, Planet Space Sci., 30, 773

Hunten, D. M., \& Watson, A. J. 1982, Icarus, 51, 655

Johnson, R. E. 2009, Phil. Trans. R. Soc. A, 367, 753

Johnson, R. E. 2010, ApJ, 716, 1573

Johnson, R. E., et al. 2008, Space Sci. Rev., 139, 355

Johnson, R. E., Tucker, O. J., Michael, M., Sittler, E. C., Waite, J. H., \& Young, D. A. 2009, in Titan from Cassini-Huygens, ed. R. H. Brown et al. (Tucson, AZ: Univ. Arizona Press), 373

Krasnopolsky, V. A. 1999, J. Geophys. Res., 104, 5955

McNutt, R. L. 1989, Geophys. Res. Lett., 16, 1225

Murray-Clay, R. A., Chiang, E. I., \& Murray, N. 2009, ApJ, 693, 23

Parker, E. N. 1964a, ApJ, 139, 72

Parker, E. N. 1964b, ApJ, 139, 93

Sone, Y., \& Sugimoto, H. 1993, Phys. Fluids A, 5, 1491

Strobel, D. F. 2008a, Icarus, 193, 612

Strobel, D. F. 2008b, Icarus, 193, 588

Strobel, D. F. 2009, Icarus, 202, 632

Tenishev, V., Combi, M., \& Davidsson, B. 2008, ApJ, 685, 659

Tian, F. 2009, ApJ, 703, 905

Tian, F., Kasting, J. F., Liu, H.-L., \& Roble, R. G. 2008, Geophys. Res., 113, E05008

Tian, F., \& Toon, O. B. 2005, Geophys. Res. Lett., 32, L18201

Tucker, O. J., Erwin, J. T., Volkov, A. N., Cassidy, T. A., \& Johnson, R. E. 2011, in AIP Conf. Proc. 1333, 27th Int. Symp. on Rareified Gas Dynamics (Melville, NY: AIP), in press

Tucker, O. J., \& Johnson, R. E. 2009, Planet Space Sci., 57, 1889

Volkov, A. N., Tucker, O. J., Erwin, J. T., \& Johnson, R. E. 2011, Phys. Fluids, submitted

Watson, A. J., Donahue, T. M., \& Walker, J. C. G. 1981, Icarus, 48, 150

Westlake, J. H., Bell, J. M., Waite, J. H., Johnson, R. E., Luhmann, J. G., Mandt, K. E., Magee, B. A., \& Rymer, A. M. 2011, J. Geophys. Res., in press Yelle, R. V. 2004, Icarus, 170, 167 


\title{
Thermally driven escape from Pluto's atmosphere: A combined fluid/kinetic model
}

\author{
O.J. Tucker*, J.T. Erwin, J.I. Deighan, A.N. Volkov, R.E. Johnson \\ Engineering Physics, 395 McCormick Road, University of Virginia, Charlottesville, VA 22904, USA
}

\section{A R T I C L E I N F O}

\section{Article history:}

Received 9 May 2011

Revised 11 November 2011

Accepted 14 November 2011

Available online 25 November 2011

\section{Keywords:}

Pluto

Atmospheres, Dynamics

Atmospheres, Structure

Aeronomy

\begin{abstract}
A B S T R A C T
A combined fluid/kinetic model is developed to calculate thermally driven escape of $\mathrm{N}_{2}$ from Pluto's atmosphere for two solar heating conditions: no heating above $1450 \mathrm{~km}$ and solar minimum heating conditions. In the combined model, one-dimensional fluid equations are applied for the dense part of the atmosphere, while the exobase region is described by a kinetic model and calculated by the direct simulation Monte Carlo method. Fluid and kinetic parts of the model are iteratively solved in order to maintain constant total mass and energy fluxes through the simulation region. Although the atmosphere was found to be highly extended, with an exobase altitude at $\sim 6000 \mathrm{~km}$ at solar minimum, the outflow remained subsonic and the escape rate was within a factor of two of the Jeans rate for the exobase temperatures determined. This picture is drastically different from recent predictions obtained solely using a fluid model which, in itself, requires assumptions about atmospheric density, flow velocity and energy flux carried away by escaping molecules at infinity. Gas temperature, density, velocity and heat flux versus radial distance are consistent between the hydrodynamic and kinetic model up to the exobase, only when the energy flux across the lower boundary and escape rate used to solve the hydrodynamic equations is obtained from the kinetic model. This limits the applicability of fluid models to atmospheric escape problems. Finally, the recent discovery of $\mathrm{CO}$ at high altitudes, the effect of Charon and the conditions at the New Horizon encounter are briefly considered.
\end{abstract}

Published by Elsevier Inc.

\section{Introduction}

Our understanding of Pluto's $\mathrm{N}_{2}$ dominated atmosphere is largely based on rare occultation observations in 1988 (pre-perihelion), 2002 and 2006 (post-perihelion) (Elliot et al., 2007; Young et al., 2008). Each observation determined the surface pressure to be between 6.5 and $24 \mu$ bars with a peak atmospheric temperature of $\sim 100 \mathrm{~K}$ at a radial distance $\sim 1250 \mathrm{~km}$. At this temperature and due to Pluto's low gravitational energy, e.g. $0.007 \mathrm{eV} / \mathrm{amu}$ at $1250 \mathrm{~km}$, escape can result in significant atmospheric loss. Therefore, many studies of Pluto's evolution have been aimed at understanding the loss rate over time (Hubbard et al., 1990; Hunten and Watson, 1982; Strobel, 2008a; Tian and Toon, 2005; Trafton, 1980).

Using results from the occultation observations and calculations of solar heating rates in continuum models of the upper atmosphere, a series of authors calculated thermal escape rates from Pluto's atmosphere by considering a process referred to as slow hydrodynamic escape (SHE, e.g., McNutt, 1989; Krasnopolsky, 1999; Strobel, 2008a). The SHE model of the atmosphere is based on the premise that thermal energy of molecules is efficiently

\footnotetext{
* Corresponding author.

E-mail addresses: ojt9j@virginia.edu (O.J. Tucker), jte2c@virginia.edu (J.T. Erwin), jid7v@virginia.edu (J.I. Deighan), av4h@virginia.edu (A.N. Volkov), rej@virginia.edu (R.E. Johnson).
}

converted into bulk flow energy. This assumption can lead to an over-estimate of the escape rate (Johnson, 2010; Volkov et al., $2011 \mathrm{a}, \mathrm{b})$ when applied to the rarefied region of the atmosphere where the collisions are too infrequent to maintain local thermal equilibrium. Using a combined fluid/kinetic model to directly account for the non-equilibrium nature of the gas flow in the upper atmosphere, thermally driven escape from Pluto's atmosphere is found to occur on a molecule by molecule basis resulting in an enhanced Jeans escape rate (Tucker et al., 2011).

Globally averaged escape rates typically have been estimated using fluid (Strobel, 2008a,b) or kinetic models (Tucker and Johnson, 2009; Volkov et al., 2011a,b). Kinetic models can in principle be applied to the entire atmosphere, but they are computationally expensive when applied to a dense region of the atmosphere. In the dense region of the atmosphere where collisions are frequent the flow can be treated as a continuum and the use of a fluid model is most efficient. In the fluid models the hydrodynamic equations are solved to obtain the mass flow rate through the atmosphere but are unable to account for relatively infrequent collisions of upwardly moving molecules or returning molecules that regulate escape in the upper atmosphere. Therefore, they cannot correctly calculate the amount of heat transported through the atmosphere. A combined fluid/kinetic model applied to dense and rarefied parts of the atmosphere respectively avoids these difficulties and provides a computationally-efficient tool for simulation of the atmosphere (e.g. Marconi et al., 1996). 
The aim of the present paper is to obtain the globally averaged escape rate, gas density, temperature, velocity, and the heat flux in Pluto's atmosphere in the region between $1450 \mathrm{~km}$ and 30,000 km using a combined fluid/kinetic model. We numerically solve the one-dimensional (1D) hydrodynamic equations coupled to a molecular kinetic model for the rarefied region of the atmosphere similar to the approach used in Marconi et al. (1996). Preliminary results were given in Tucker et al. (2011). Here simulations are performed for no heating and solar minimum heating conditions. The interaction of Pluto's extended atmosphere with Charon and the implications of the recently discovered CO detection in Pluto's extended atmosphere (Greaves et al., 2011) are briefly considered. The results presented here also suggest that the application of the hydrodynamic models to escape from other planetary atmospheres (e.g., Murray-Clay et al., 2009; Tian, 2009; Strobel, 2008 a,b) can give incorrect estimates of the macroscopic properties and the escape flux.

\section{Jeans, hydrodynamic, and slow hydrodynamic escape}

Although escape driven by solar heating is by its nature a threedimensional (3D) process, for comparison with previous models, the thermal escape problem is formulated here by considering a 1D, globally averaged, steady-state model of the atmosphere, where the gas properties are functions of the radial distance $r$, from the planet center. In this section, the applicability of the fluid model to thermal escape is briefly analyzed. Based on this analysis, a combined fluid/kinetic model is introduced in the next section.

The Jeans parameter, $\lambda(r)$, the ratio of the gravitational energy of a molecule $\Phi_{\mathrm{g}}=G M_{\mathrm{p}} m / r$ to its thermal energy $k T$, is often used to characterize the atmospheric escape rate: i.e., $\lambda(r)=\Phi_{\mathrm{g}} / k T$, with $G$ the gravitational constant, $M_{\mathrm{p}}$ the planet mass, $m$ the molecular mass, $k$ the Boltzmann constant and $T$ the temperature at $r$. In order to escape a planet's gravity a molecule must be directed outward from the planet, have a velocity larger than $v_{\mathrm{esc}}=\sqrt{2 G M_{\mathrm{p}} / r}$ and have a low probability of colliding with other molecules along its trajectory. The density of planetary atmospheres decreases exponentially with altitude and the most rarefied region is referred to as the exosphere. In this region intermolecular collisions are rare, therefore the Jeans parameter is typically evaluated at the lower boundary of the exosphere which is referred to as the exobase $r_{x}$. Throughout the paper the subscript " $x$ " will be used to denote the values of all parameters evaluated at $r=r_{x}$.

The degree of rarefaction of a gas is determined by the local Knudsen number, $K n=l_{c} / l_{a}$, the ratio of the mean free path of the molecules, $l_{c} \sim c /(n \sigma)$, to an appropriate length scale for the gas density $l_{a}$. Here $\sigma$ is the molecular cross section, $n$ is the local number density, and the numerical coefficient $c$ depends on the model of intermolecular collisions, e. g., for hard sphere molecules $c=1 / \sqrt{ } 2$ (Bird, 1994; Chapman and Cowling, 1970). The appropriate length $l_{a}$ for planetary atmospheres is usually defined as the distance over which the density decreases by a factor of $1 / e$ and is called the atmospheric scale height $H=r / \lambda(r)$, so that $K n=l_{c} / H$. When $K n \ll 0.1$ the atmosphere is relatively dense so that molecules collide frequently. With increasing altitude collisions become less frequent and the exobase altitude is defined to occur where $K n_{x} \sim 1$ or $n_{x} H_{x} \sigma \sim 1$.

Three regimes of escape are typically characterized using the Jeans parameter. If a planetary atmosphere has a relatively large Jeans parameter at the exobase for the dominant atmospheric species, thermal escape occurs on a molecule by molecule basis similar to evaporation, a process referred to as Jeans escape (e.g., Chamberlian and Hunten, 1987; Jeans, 1916). In this approximation it is assumed the speed distribution at the exobase is Maxwellian, so that the molecular loss rate is $\varphi_{\mathrm{J}}=\pi r_{x}^{2} n_{x}\left\langle v_{\mathrm{th}, x}\right\rangle\left(1+\lambda_{x}\right) \exp \left(-\lambda_{x}\right)$, where $\left\langle v_{\mathrm{th}, x}\right\rangle=\left(8 \mathrm{k} T_{x} / \pi \mathrm{m}\right)^{1 / 2}$ is the mean thermal speed of molecules at the exobase. The concomitant cooling rate, total energy of molecules escaping the atmosphere per unit time, is $\langle E \varphi\rangle_{\mathrm{J}}=\left(k T_{x}\right)(2+1 /$ $\left.\left(1+\lambda_{x}\right)\right) \varphi_{\mathrm{J}}$. Modified Jeans escape rates, accounting for the non-zero gas velocity at the exobase, have also been proposed (e. g. Chamberlain, 1961; Yelle, 2004; Volkov et al., 2011a,b).

At small Jeans parameters the thermal energy of molecules is comparable to or larger than the gravitational binding energy of the planet at the exobase, so the bulk atmosphere can escape as a hydrodynamic outflow (e.g., Öpik, 1963; Hunten, 1973; Volkov et al., 2011a; Gruzinov, 2011). This is often referred to as blow off resulting in escape rates much larger than the Jeans rate. Blow off has been suggested to occur when $\lambda_{x} \lesssim 2$ at the exobase altitude or below (Hunten, 1973; Watson et al., 1981).

The slow hydrodynamic escape (SHE) model, considered intermediate to Jeans and hydrodynamic escape regimes, has been suggested to be applicable to a dense tightly bound atmosphere for which the Jeans parameter, $\lambda\left(r_{0}\right)$ is larger than 10 , estimated at a radial distance, $r_{0}$, considered to be in approximate thermal and radiative equilibrium (e.g., Parker, 1964b; Watson et al., 1981). The flow is referred to as slow because near $r_{0}$ the gravitational energy $\Phi_{\mathrm{g}}$ dominates the thermal energy $\left(C_{\mathrm{p}} T\right)$, which also dominates the flow energy $\left(1 / 2 m u^{2}\right)$ where $C_{\mathrm{p}}$ is the heat capacity per molecule and $u(r)$ is the flow speed. However the flow eventually reaches supersonic speeds above the exobase resulting in escape rates much larger than the Jeans rates. Below we provide a more detailed discussion on the application of hydrodynamic models to the slow hydrodynamic escape regime.

In the 1D steady-state hydrodynamic model the continuity equation leads to a constant molecular flow, given here as a flow rate, $\varphi$, versus radial distance:

$\varphi=4 \pi r^{2} n(r) u(r)=4 \pi r_{0}^{2} n\left(r_{0}\right) u\left(r_{0}\right)$

The radial momentum equation, in which the viscous term is dropped, is:

$\frac{d p}{d r}=n\left(\frac{d \Phi_{g}}{d r}-\frac{d}{d r}\left(\frac{1}{2} m u^{2}\right)\right)$

with the gas pressure $p=n k T$. Finally, the corresponding energy equation is:

$\frac{d}{d r}\left\{\varphi\left(\frac{1}{2} m u^{2}+C_{\mathrm{p}} T-\Phi_{\mathrm{g}}\right)-4 \pi r^{2} \kappa(T) \frac{d T}{d r}\right\}=4 \pi r^{2} Q(r)$

where $\kappa(T)$, is the thermal conductivity, $C_{\mathrm{p}}$ is the heat capacity per molecule and $Q(r)$ accounts for the solar heating and IR cooling rates. Knowing the number density, $n_{0}$, and temperature, $T_{0}$, at the lower boundary, i.e. $n\left(r_{0}\right)=n_{0}, T\left(r_{0}\right)=T_{0}$, Eqs. (1b) and (1c) are solved. A unique solution requires two additional parameters at the lower boundary, $u_{0}$ (or $\varphi$ ) and $(d T / d r)_{0}$. Unfortunately, in order to find $u_{0}$ and $(d T / d r)_{0}$, one needs to impose assumptions about the solution behavior at $r \rightarrow \infty$.

Parker (1958) used the hydrodynamic equations to model the thermal expansion of the solar wind in the vicinity of $\lambda\left(r_{0}\right) \sim 2$. He subsequently extended that model to describe the expansion of a stellar wind from a star with a tightly bound corona with $\lambda\left(r_{0}\right) \gtrsim 10$ for which no or very little heat is deposited above $r_{0}$ (Parker, 1964a,b). In this formulation, escape is powered by the heat flow from below $r_{0}$ and the conditions imposed are $T, n \rightarrow 0$ as $r \rightarrow \infty$. It was then shown that the dense atmosphere must expand according to a critical solution, where the flow velocity, $u$, gradually increases above the isothermal speed of sound. Purely subsonic solutions were not permitted because they resulted in a finite pressure at infinity.

Chamberlain $(1960,1961)$ re-considered the expansion of the solar wind for subsonic velocities with the condition $T \rightarrow 0$ as $r \rightarrow \infty$. He deemed this approach to be a slow hydrodynamic expansion of the solar wind, and showed it is possible to obtain a 
subsonic solution with the hydrodynamic equations if the energy flux at infinity is 0 . In this formulation the number density $n$ approaches a constant as $r \rightarrow \infty$. Later, Parker (1964b) acknowledged this result as a limiting case to supersonic expansion. He determined that this approximation would only occur in the limit that the density at the lower boundary, $n_{0}$, goes to infinity. He showed for sufficiently large densities at $r_{0}$, the energy flux carried to infinity is non-zero for the condition $T \rightarrow 0$ and, hence, the expansion can proceed supersonically. This is the typical approach used in applying the SHE model to planetary atmospheres (e.g., Krasnopolsky, 1999; Strobel, 2008a,b; Watson et al., 1981). Since the flow is slow, the standard procedure is to integrate Eqs. (1b) and (1c) neglecting the $u^{2}$ terms (Parker, 1964b). Therefore, although $\varphi \neq 0$ in Eq. (1a), $u^{2}$ is set equal to 0 in Eqs. (1b) and (1c) below an upper boundary where $1 / 2 m u^{2} \ll C_{\mathrm{p}} T$, and $T$ and $n$ are only regarded as valid out to an $r$ where the $u$ is a small fraction of the local sound speed (McNutt, 1989; Krasnopolsky, 1999; Strobel, 2008a,b).

The SHE model has been subsequently applied to the thermal expansion of planetary atmospheres in which solar EUV and UV heating powers escape above $r_{0}$ (Watson et al., 1981). Particular emphasis has been placed on Pluto's atmosphere which is widely thought to be escaping hydro-dynamically (e.g., McNutt, 1989; Krasnopolsky, 1999; Strobel, 2008a; Tian and Toon, 2005). For example McNutt (1989) solved the hydrodynamic equations neglecting the term $C_{\mathrm{p}} T$ in Eq. (1c) to obtain an analytical solution for $n, T$, and the escape rate $\varphi$, assuming the solar heating occurred in a narrow region of the atmosphere. Krasnopolsky (1999) retained the $C_{\mathrm{p}} T$ term, using numerical methods to solve the hydrodynamic equations.

More recently Strobel (2008a,b) applied the SHE model to the atmospheres of Titan and Pluto using more realistic lower boundary conditions at a radial distance where the atmosphere is in approximate radiative equilibrium. He iteratively solved Eqs. (1b) and (1c) using assumed values of $\varphi$ and $(d T / d r)_{0}$, to find a solution with the right asymptotic behavior and zero total energy flux at $r \rightarrow \infty$ for Pluto (Strobel, 2008a) and matched to available density data for Titan (Strobel, 2008b). Calculated $\mathrm{N}_{2}$ escape rates from Pluto, $\varphi \sim 9.4 \times 10^{26} \mathrm{~s}^{-1}$, and Titan, $\varphi \sim 1.5 \times 10^{27} \mathrm{~s}^{-1}$, were several orders of magnitude larger than the Jeans escape rates calculated using the corresponding SHE model exobase densities and temperatures. For example, the most recent SHE model estimate for escape from Pluto's atmosphere is $\sim 10^{3}$ times the Jeans rate for the suggested atmospheric structure at solar minimum conditions (Strobel, 2008a).

Tucker and Johnson (2009) tested the results for Titan using a kinetic approach and did not obtain large escape rates. In fact, when the temperature in Titan's upper atmosphere was artificially increased so that $\lambda_{x} \sim 11$, similar to that at Pluto, the escape rate obtained was enhanced over the Jeans rate but only by a factor of 1.5. Using a kinetic model, Volkov et al. (2011a,b) showed that thermal escape rate from both monatomic and diatomic atmospheres, for which most of the heating occurs below $r_{0}$, differs from the Jeans rate by less than a factor of 2 if $\lambda\left(r_{0}\right) \gtrsim 6$. Such a drastic difference between fluid and kinetic simulations in the domain of the slow hydrodynamic escape, $\lambda\left(r_{0}\right) \gtrsim 10$, is due to the incorrect treatment of the rarefied region of the atmosphere in the hydrodynamic approximation.

The discrepancy between results obtained from solving the hydrodynamic equations and kinetic simulations can be resolved by using a combined fluid and kinetic approach (e.g., Marconi et al., 1996). A stand-alone kinetic simulation is computationally infeasible at $K n\left(r_{0}\right) \sim 10^{-6}$ characteristic of the density at the lower boundary typically used in modeling escape from Pluto's atmosphere. Therefore, a computationally efficient model can be constructed by coupling the hydrodynamic equations for the dense atmosphere with kinetic simulations for the exosphere region.

\section{Combined fluid/kinetic model of thermal escape}

A fluid/kinetic model is applied from a lower boundary $r_{0}$ in the atmosphere, considered to be in approximate local thermodynamic equilibrium, to a top boundary $r_{1}$ where the atmospheric flow is essentially free of collisions. We divide the atmosphere into two regions, a fluid region where the hydrodynamic equations are applicable from $r_{0}$ where $K n \ll 1$ to an intermediate boundary $r_{\text {od }}$ chosen to correspond to $K n \sim 0.1$, and a kinetic region where kinetic simulations are performed by means of the direct simulation Monte Carlo (DSMC) method (Bird, 1994), from $r_{\text {od }}$ to $r_{1}$ where $K n \gg 1$.

When solving the fluid equations we make no assumptions about the $n$ and $T$ at infinity. Consistent with the SHE model we drop the $u(r)^{2}$ terms. It is possible to include such terms in both the SHE and fluid/kinetic approaches. However, for a dense gravitationally bound atmosphere $u(r)^{2}$ can be safely neglected below the exobase (Parker, 1964b). The lower boundary conditions in the fluid region are $n_{0}$ and $T_{0}$, while the parameters $\varphi$ and $\langle E \varphi\rangle_{r_{0}}$, the particle and energy flow across $r_{0}$, in Eq. (2b) are determined by the DSMC part of the model. The pressure and the heat flow, given by Eqs. (2a) and (2b), are determined from the integration of Eqs. (1b) and (1c) using the total heating rate $\beta(r)=r_{0}^{-2}\left[\int_{\mathrm{r} 0} r^{2} Q(r) d r\right]$ with $\beta \rightarrow \beta_{0}$ as $r \rightarrow \infty$, as defined in Strobel (2008a), and $\langle E \varphi\rangle_{r_{0}}$ is obtained as the constant of integration:

$$
\begin{aligned}
& p=p_{0} \exp \left[-\int \frac{\lambda(r)}{r} d r\right] \\
& \varphi\left(C_{\mathrm{p}} T-\Phi_{\mathrm{g}}\right)-4 \pi r^{2} \kappa(T) \frac{d T}{d r}=\langle E \varphi\rangle_{r_{0}}+4 \pi r_{0}^{2} \beta(r)
\end{aligned}
$$

Since the fluid model requires initial values for $\varphi$ and $\langle E \varphi\rangle_{\mathrm{r}_{0}}$, for the $\beta_{0}=0$ case we began by assuming an isothermal, hydrostatic atmosphere, $d\left(n k T_{0}\right) / d r=n\left[d \Phi_{g} / d r\right]$, and used a DSMC simulation for such an atmosphere starting at $K n\left(r_{\text {od }}\right) \sim 0.1$ to obtain the initial estimates. Therefore unlike the SHE model we do not assume that the energy flow at infinity is zero. In a steady state atmosphere energy conservation requires that the energy carried off by escaping molecules is replaced by a flow of energy into the lower boundary, $\langle E \varphi\rangle_{r_{0}}$, with $\langle E \varphi\rangle=\langle E \varphi\rangle_{r_{0}}+4 \pi r_{0}^{2} \beta_{0}$. With such starting conditions, the fluid/kinetic model typically obtained a converged solution in four iterations. That is, the temperatures agree within $<3 \%$ and densities agree within $<2 \%$ in the region where $0.1<K n<1$.

As schematically presented in Fig. 1 we solve Eqs. (2a) and (2b) for the density and temperature up to the exobase and iteratively obtain $\varphi$ and $\langle E \varphi\rangle_{r_{0}}$. From that solution the resulting $n_{\text {od }}$ and $T_{\text {od }}$ at a radius $r_{\text {od }}$ where $K n \sim 0.1$, which is about two scale heights below the nominal exobase, are used in the DSMC simulation up to an altitude many scale heights above the exobase, $K n \gg 1$. The DSMC method tracks a representative sample of atmospheric molecules which are under the gravitational influence of Pluto and subjected to mutual collisions. At the upper boundary of the kinetic domain we obtain the particle escape rate, $\varphi$, and the energy flow through the 1D system, $\langle E \varphi\rangle$. Values of $\langle E \varphi\rangle$ and $\varphi$ from the upper boundary are used to update corresponding values in the fluid part of the model and then used to solve Eqs. (2a) and (2b) up to $K n=1$ during the next iteration. Likewise the results from the new simulation of the hydrodynamic equations provide updated $n_{\text {od }}$ and $T_{\text {od }}$ at $K n\left(r_{\text {od }}\right) \sim 0.1$ for the kinetic model, which are then used to obtain new values of $\varphi$ and $\langle E \varphi\rangle$. This procedure is repeated until we reach consistent densities, temperatures and flow velocities in the region where the fluid and kinetic model overlap. That is, for $K n \leqslant 1$ we numerically solve Eqs. (2a) and (2b) to obtain $n(r)$ and $T(r)$. The flow velocity, $u(r)$, is given by Eq. (1a) for the set of escape parameters $\varphi$ and $\langle E \varphi\rangle$ obtained from the DSMC simulations. We consider a converged solution acceptable when the temperatures 


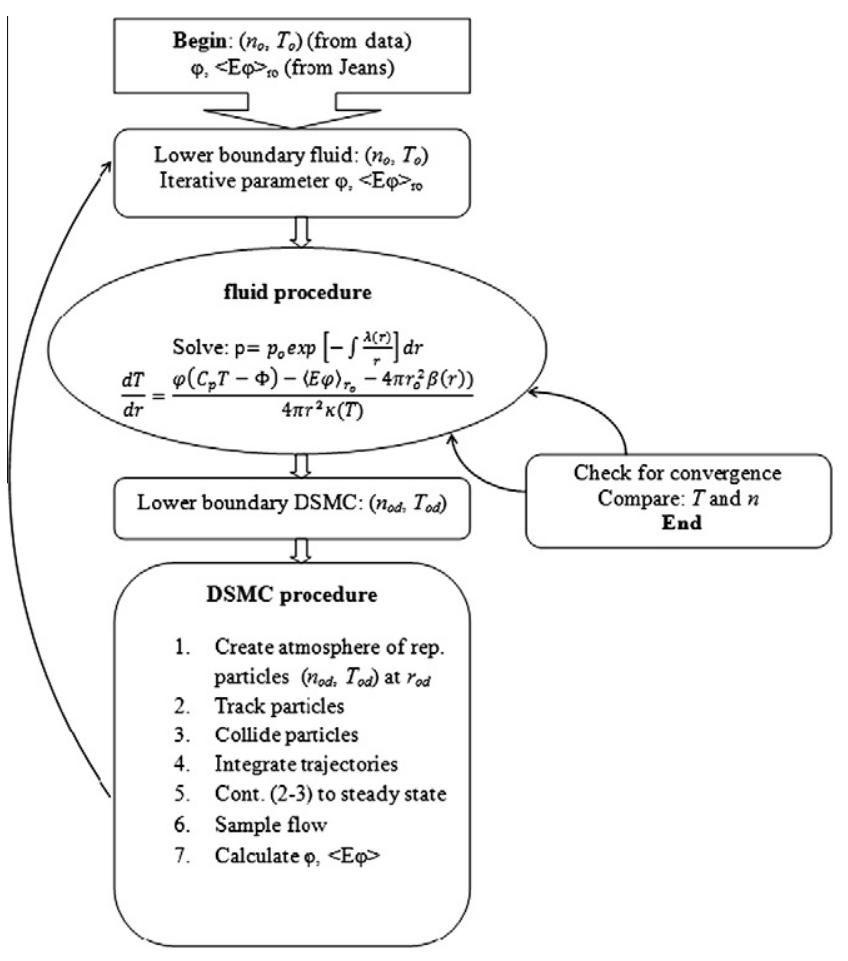

Fig. 1. Schematic of the numerical implementation of the fluid/kinetic model. To obtain solutions of the hydrodynamic equations at $r_{0}$ where $K n<0.1, n_{0}$ and $T_{0}$ are given, and $\varphi$ and $\langle E \varphi\rangle_{r_{0}}$ are obtained iteratively using the DSMC method. An initial guess of the energy flow into the lower boundary $\langle E \varphi\rangle_{r_{0}}$ is used to solve Eqs. (2a) and ( $2 \mathrm{~b}$ ) up to $K n \sim 1$. From the fluid solution $n_{\text {od }}$ and $T_{\text {od }}$ calculated at $K n_{\text {od }} \sim 0.1$ are used in the DSMC simulation up to $K n \gg 0.1$ to obtain updated $\varphi$ and $\langle E \varphi\rangle_{\text {r }}$. The iterations are continued until temperature, density and heat flux are consistent between the fluid and DSMC solutions in the overlap region of $0.1<K n<1$.

and densities agree within $₹ 3 \%$ in the overlap region between our fluid and kinetic models where $0.1<K n<1$.

In the low Knudsen number regime Eqs. (2a) and (2b) were solved simultaneously using a 4th order Runge-Kutta method with the adaptive radial step-size Runge-Kutta-Fehlberg method (Burden and Faires, 2005), to ensure a relative tolerance of $10^{-8}$ for $n(r)$ and $T(r)$. The integration steps were between 0.1 and $2 \mathrm{~km}$ with the finer resolution necessary to resolve the faster change in temperature near the lower boundary and the narrow heating peak. The heating/cooling models described in Strobel (2008a) are used for the solar heating due to $\mathrm{N}_{2}$ and $\mathrm{CH}_{4}$ absorption bands (including UV, EUV, and near-IR) and CO radiative cooling. Since the heating and cooling rates depend on the temperature and column of gas above a given radial position in the atmosphere, the spatial distribution in the net heating rate is recalculated using the new density profile. These iterations are performed until energy conservation is achieved between $\langle E \varphi\rangle_{r_{0}}, \beta_{0}$, and $\langle E \varphi\rangle$.

The DSMC method applied in the kinetic region $K n>0.1$ in effect solves the Boltzmann kinetic equation by the directly modeling the stochastic nature of the molecular motion in the gas flow using Monte Carlo techniques (Bird, 1994). The DSMC method uses a set of modeling molecules in order to calculate the gas properties of the atmosphere at a molecular level. Collisions between molecules are calculated in discrete radial cells based on the local values of the relative speed, cross section and density. Therefore, the DSMC method is a direct approach for describing the transition in an atmosphere from collisional to collisionless flow. In such a model the conductive heat transfer is represented microscopically.

At the lower boundary of the DSMC domain, $r_{\text {od }}$, the density $n_{\text {od }}$ and temperature $T_{\text {od }}$ are taken from the solutions of the fluid equations as discussed. Although in the DSMC simulations molecular motion and collisions are tracked in 3D, in this paper, Pluto's atmosphere is assumed to be spherically symmetric and so the resulting properties depend only on $r$, consistent with previous models for Pluto's atmosphere (Krasnopolsky, 1999; McNutt, 1989; Strobel, 2008a). Therefore, in the simulation domain when evaluating the collision probabilities the molecular velocities and positions are rotated to a common radial axis. When molecules traverse the DSMC upper boundary, those with velocities greater than the escape velocity and directed outward are assumed to escape and the others are specularly reflected. The reflected molecules represent molecules with trajectories that would eventually return to the simulation domain.

In the DSMC method the time step is chosen to be much smaller than the mean collision time and the cell widths in the flow direction are kept much smaller than $l_{c}$ and $H$ following the general recommendations in Bird (1994). We use variable cell widths in the radial direction which were approximately $1 / 3 \mathrm{rd}$ of the local mean free path and capped at $10 \%$ of the local scale height for mean free paths larger than the local atmospheric e-folding. A time step of $\sim 1-2.5 \mathrm{~s}$ provided energy conservation and ensured that every molecule would have no more than 1 collision over a time step on average. After $\sim 5 \times 10^{6} \mathrm{~s}$ the macroscopic properties of the flow were sampled for an additional $5 \times 10^{7} \mathrm{~s}$. The number of representative molecules was chosen to ensure a sufficient number of molecules $(>200)$ in the upper most cell, typically we used several $10^{3}-10^{5}$ representative molecules to describe the flow in the kinetic region. The upper boundary location was increased until the escape rate varied by less than $5 \%$ with increasing upper boundary. Likewise, when using the converged fluid solution with a DSMC lower boundary deeper in the atmosphere, i.e., choosing a point from the fluid solution between $0.01<K n\left(r_{\text {od }}\right)<0.1$, did not significantly affect the results.

Collisions between atmospheric molecules were computed using both the hard sphere (HS) model and the variable hard sphere (VHS) model (Bird, 1994). To ensure consistency between the fluid and kinetic models, we also used the Larsen-Borgnakke (LB) approximation for internal energy and the VHS cross section is parameterized to the temperature dependent thermal conductivity $\kappa(T)=\kappa_{0} T^{\omega}$ for the Maxwell gas, $\omega=1$. The VHS cross section, relevant for low speed molecular collisions, depends on the relative collision speed $v_{r}, \sigma=\sigma_{0}\left(\left\langle v_{r 0}\right\rangle /\left\langle v_{r}\right\rangle\right)$ : where $\sigma_{0}$ is a reference cross section determined from the thermal conductivity and $\left\langle v_{r 0}\right\rangle$ is the average relative velocity with both values obtained for $T_{0}$ assuming the Maxwellian speed distribution. At temperatures characteristic for Pluto's upper atmosphere, the $\mathrm{N}_{2}$ vibrational modes are assumed not to be excited so that the LB model is used only for two rotational degrees of freedom. The initial internal energy for each molecule is set at the lower boundary of the DSMC regime, $r_{\text {od }}$, based on a Maxwell-Boltzmann energy distribution and neglecting changes in rotational levels due to IR cooling between collisions.

\section{Results for Pluto's atmosphere}

The 1D radial fluid/kinetic model is applied to a region in Pluto's atmosphere from $r_{0}=1450 \mathrm{~km}$ up to $r_{1}=30,000 \mathrm{~km}$ for $n_{0}=4 \times 10^{12} \mathrm{~cm}^{-3}\left(K n_{0} \sim 10^{-6}\right)$ and $T_{0}=88.2 \mathrm{~K}$ consistent with Strobel (2008a). Pluto's orbital axis is nearly parallel to its orbital plane which will result in the structure of the atmosphere being non-isotropic over the globe, and the amount of solar heating is also variable dependent upon the relative abundances of $\mathrm{CH}_{4}$ and $\mathrm{CO}$ present in the atmosphere. However, for the purpose of this study we assume a globally averaged atmosphere and adopted solar minimum heating rates from Strobel (2008a) to compare with the SHE model results. Further studies should be done using a 3D model as the amounts of $\mathrm{CH}_{4}$ and $\mathrm{CO}$ in the atmosphere are better constrained. 
We first obtained a converged solution for density and temperature versus radial distance with the fluid/kinetic approach using the HS collision model with the DSMC simulation. To compare the effects of using the HS, HS-LB, VHS and VHS-LB collision models on $\varphi, n(r), T(r)$ and $u(r)$ within the DSMC model, the following lower boundary conditions were adopted from the converged fluid/kinetic (HS) model $r_{\text {od }}=2836 \mathrm{~km}$ where $\lambda_{\text {od }}=12, n_{\text {od }}=2.9 \times 10^{7} \mathrm{~cm}^{3}$ and $T_{\text {od }}=85.5 \mathrm{~K}$. Using the conductivity for the Maxwell molecules at $T_{0}$ (Strobel, 2008a),we obtained a reference value for the HS cross section of $\sigma_{0}=9.0 \times 10^{-15} \mathrm{~cm}^{2}$, see Table 1 for the model parameters. The mean free paths at the lower boundary for a collision for HS and VHS molecules are $l_{c}=\left(\sqrt{ } 2 n \sigma_{0}\right)^{-1}$ and $l_{c}=\left(\left\langle v_{\mathrm{th}}\right\rangle / n \sigma_{0}\left\langle v_{\mathrm{r} 0}\right\rangle\right)$ respectively (Bird, 1994), but the values of $l_{c}$ and $K n\left(r_{\text {od }}\right)$ for this particular case are similar: $\sim 27 \mathrm{~km}$ and $\sim 0.1$ respectively. While the resulting density profiles did not significantly depend on the choice of the collision model for the parameters $n_{\text {od }}$ and $\lambda_{\text {od }}$ given above (e.g. for all results $r_{x} \sim 3900 \mathrm{~km}$ ), the resulting temperature profiles and escape rates were slightly different: e.g., the escape rates are 4.4, 5.1, 4.3 and $4.8 \times 10^{25} \mathrm{~s}^{-1}$ for the HS, HS-LB, VHS and VHS-LB models respectively. Above the exobase, as it is seen in Fig. 2a the translational temperature decreases faster than the rotational temperature, and the perpendicular temperature decreases faster than the radial temperature. At distances increasingly above the exobase the atmosphere cools approximately adiabatically as collisions become increasingly infrequent.

In the transition region of the atmosphere there is an altitude where it is most efficient for molecules to acquire escape trajectories. Below this altitude collisions inhibit escape and above there are too few collisions to produce escape trajectories. In the kinetic region we calculated the average number of escaping molecules produced in each radial cell, $d \varphi / d r_{\text {esc }}$, by noting the altitude at which molecules that eventually traverse the top of the simulation domain, $r_{1}=30,000 \mathrm{~km}$, first attained an escape velocity. Molecules that later lose their escape velocity are dropped from the inventory, so the total escape rate is given by $\varphi=\int_{r_{0}}^{r_{1}}(d \varphi / d r)_{e s c} d r$. The peak in the escape rate production, Fig. $2 \mathrm{~b}$, occurs at the same altitude for the HS $(r \sim 4090 \mathrm{~km})$ and VHS $(r \sim 3680 \mathrm{~km})$ models with and without the internal degrees of freedom. The difference in the peak altitude is determined by the conductivity which differs between the VHS $(\kappa \propto T)$ and HS models $\left(\kappa \propto T^{1 / 2}\right)$. For the fluid/kinetic results discussed further below we used the VHS-LB model in order to have both $\kappa(T)$ and $C_{\mathrm{p}}$ consistent with the fluid model, and to allow for rotational/ translation energy exchange.

Results are given in Table 2 from two cases obtained using the combined fluid/kinetic simulation with the VHS-LB collision model. In the first case no solar heating occurs in the simulation region: i.e., $Q(r)=0$ above $r_{0}$ so that $\beta_{0}=0$. Next we assume approximate solar minimum conditions where the net heating/cooling above $r_{0}$ is such that $\beta_{0}=1.5 \times 10^{-3} \mathrm{erg} \mathrm{cm}^{-2} \mathrm{~s}^{-1}$ which is similar to the value used in the SHE model $\beta_{0}=1.7 \times 10^{-3} \mathrm{erg} \mathrm{cm}^{-2} \mathrm{~s}^{-1}$ (Strobel, 2008a). The value of $\beta_{0}$ was obtained by using a fixed solar UV and EUV heating efficiency, $\varepsilon \sim 0.25$, with a cut-off in the heating at an altitude where the heat deposited was less than $1 \%$ of $\beta_{0}$ (Strobel, 2008a).

Table 1

Parameters for fluid/kinetic model.

\begin{tabular}{lll}
\hline Parameter & HS model & VHS model \\
\hline Heat capacity/molecule: $C_{\mathrm{p}}$ & $(3 / 2) k$ & $(5 / 2) k$ \\
Viscosity exponent: $\omega\left(\kappa(T)=\kappa_{0} T^{\omega}\right)$ & $1 / 2$ & $1^{\mathrm{a}}$ \\
Collision cross section: $\sigma\left(\times 10^{-15}\right) \mathrm{cm}^{-2}$ & $\sigma_{0}=9.0$ & $\sigma=\sigma_{0}\left(\left\langle v_{r 0}\right\rangle / v_{r}\right)^{\mathrm{b}}$ \\
\hline
\end{tabular}

Parameters for $\mathrm{N}_{2}$ used in the fluid/kinetic. The lower boundary radial distance is $r_{0}=1450 \mathrm{~km}$ where $n\left(r_{0}\right)=4 \times 10^{12} \mathrm{~cm}^{-3}\left(K n_{0} \sim 10^{-6}\right), T\left(r_{0}\right)=88.2 \mathrm{~K}$.

a The viscosity exponent for the VHS model and $\kappa_{0}=9.37 \mathrm{erg} \mathrm{cm}^{-1} \mathrm{~s}^{-2} \mathrm{~K}^{-2}$ are taken from Strobel (2008a).

b The average relative velocity at $r_{0}$ is defined by $\left\langle v_{r 0}\right\rangle=\left(16 k T_{0} / \pi m\right)^{1 / 2}$.
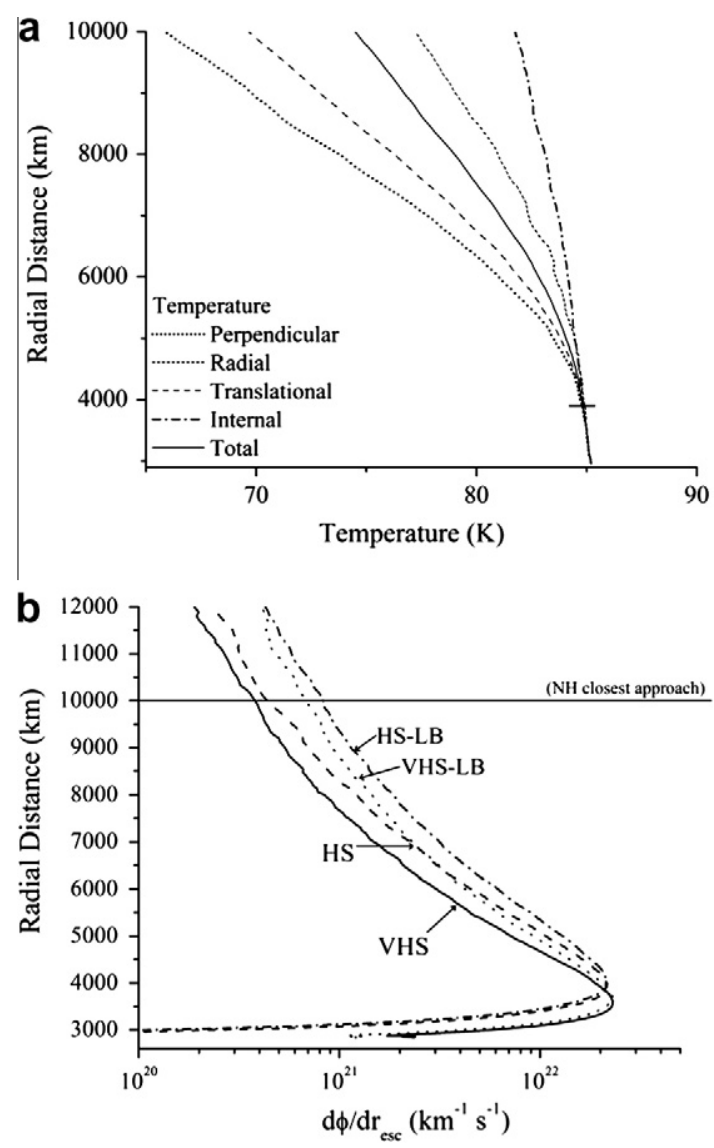

Fig. 2. Results of test simulation described in text $K n\left(r_{\text {od }}\right)=0.1$ and $\beta(r)=0$ above lower boundary: exobase altitude is $3900 \mathrm{~km}$ indicated by short horizontal curve in Fig. 2a: (a) $T$ (K) in VHS-LB model: short dashed curve perpendicular temperature, $T_{\text {perp }}$, dotted curve radial temperature, $T_{\mathrm{r}}$, dashed dotted curve rotational temperature, $T_{\text {rot }}$, dashed curve translational temperature, $T_{\text {trans }}=\left(T_{\mathrm{r}}+2 T_{\text {perp }}\right) / 3$, solid curve total temperature, $T=\left(3 T_{\text {trans }}+2 T_{\text {rot }}\right) / 5$. (b) Production of escaping molecules, $d \varphi /$ $d r_{\text {esc }}\left(\mathrm{km}^{-1} \mathrm{~s}^{-1}\right)$ versus $r$ : HS (dashed curve), VHS (solid curve), HS-LB (dashed dotted curve), VHS-LB (dotted curve). The New Horizons spacecraft distance of closest approach to Pluto will be $10,000 \mathrm{~km}$.

Table 2

SHE versus fluid/kinetic.

\begin{tabular}{|c|c|c|c|c|}
\hline \multirow[t]{2}{*}{$\beta_{0}\left(10^{-3} \mathrm{erg} \mathrm{cm}^{-2} \mathrm{~s}^{-1}\right)$} & \multicolumn{2}{|c|}{ No heating } & \multirow{2}{*}{$\begin{array}{l}1.7 \\
\text { SHE }\end{array}$} & \multirow{2}{*}{$\begin{array}{l}1.5 \\
\text { Fluid/kinetic }\end{array}$} \\
\hline & $\mathrm{SHE}^{*}$ & Fluid/kinetic ${ }^{\mathrm{a}}$ & & \\
\hline$r_{x}(\mathrm{~km})$ & 2700 & 3900 & 3530 & 6200 \\
\hline$n_{x}\left(\times 10^{5} \mathrm{~cm}^{-3}\right)$ & 53 & 17 & 53 & 6.7 \\
\hline$T_{X}(\mathrm{~K})\left[H_{x}(100 \mathrm{~km})\right]$ & $48[1.2]$ & $85[4.5]$ & $65[2.6]$ & $87[12]$ \\
\hline$u_{x}\left(\mathrm{~m} \mathrm{~s}^{-1}\right)$ & 1 & $5 \times 10^{-4}$ & 2 & 4 \\
\hline$\lambda_{x}$ & 23 & 8.8 & 13 & 5.4 \\
\hline$\hat{\varphi}\left(10^{25} \mathrm{~s}^{-1}\right)$ & 54 & 4.8 & 180 & 120 \\
\hline$\varphi / \varphi_{\mathrm{J}}$ & $\sim 10^{7}$ & 1.6 & $\sim 10^{3}$ & 2.0 \\
\hline$\langle E \varphi\rangle / \mathrm{kT}_{0} \varphi$ & 0 & 1.8 & 0 & 1.8 \\
\hline$\langle E \varphi\rangle_{\mathrm{J}} / \mathrm{kT}_{0} \varphi_{\mathrm{J}}$ & $\sim 1.11$ & 2.02 & $\sim 1.53$ & 2.12 \\
\hline
\end{tabular}

Exobase values for the density, temperature, bulk velocity, escape rate $\varphi$ and average energy carried off $\langle E \varphi\rangle$ including the corresponding values for the theoretical Jeans escape rate and energy flow rate $\left(\varphi_{\mathrm{J}},\langle E \varphi\rangle_{\mathrm{J}}\right)$ evaluated at the corresponding exobase distances: simulations performed for $\beta_{0}=0$ and solar minimum conditions for the SHE model and the fluid/kinetic (VHS-LB) results shown in Fig. 3. The lower boundary radial distance is $r_{0}=1450 \mathrm{~km}$ where $n\left(r_{0}\right)=4 \times 10^{12} \mathrm{~cm}^{-3}$ $\left(K n_{0} \sim 10^{-6}\right), T\left(r_{0}\right)=88.2 \mathrm{~K}, \lambda\left(r_{0}\right)=23$ and $c_{0}=191 \mathrm{~m} / \mathrm{s}$ (sound speed).

* The exobase values for the SHE model are taken from Strobel (2008a).

a The exobase altitude $r_{x}$ is determined where $K n=l_{c} / H=1, l_{c}=\left\langle v_{\mathrm{th}}\right\rangle /\left(n \sigma_{0}\left\langle v_{r 0}\right\rangle\right)$.

As seen in Table 2, the escape rate obtained for the $\beta_{0}=0$ case, $4.8 \times 10^{25} \mathrm{~s}^{-1}$, is $\sim 1.6 \times \varphi_{\mathrm{J}}$, where $\varphi_{\mathrm{J}}$ is the Jeans rate for $T_{x}=85 \mathrm{~K}$ and the heat flow out is $\sim 1.4 \times\langle E \varphi\rangle_{\mathrm{J}}$ both evaluated at the 
exobase, $r_{x}=3900 \mathrm{~km}$ where $K n=1$. These results differ significantly from those obtained in the SHE model (e.g., $r_{x}=2700 \mathrm{~km}$, $n_{x} \sim 5.3 \times 10^{6} \mathrm{~cm}^{-3}, T_{x}=48 \mathrm{~K}$ and $\lambda_{x} \sim 23$; Strobel, 2008a) indicative of the very different atmospheric profiles as seen in Fig. 3a. The change in temperature with increasing $r$ is seen to fall off much faster in the SHE solution consistent with an overestimate in the adiabatic cooling due to the overestimate in the escape rate. Even though the SHE model has a much larger Jeans parameter at the exobase, the escape rate is an order of magnitude larger than that obtained with the fluid/kinetic model.

Numerically solving the fluid equations for $\beta_{0} \neq 0$ is very sensitive to the choice of the input escape parameters, especially the energy carried away by escape, $\langle E \varphi\rangle$. Therefore, an initial solution was achieved by incrementally adding in a small fraction of the heating rate and solving the fluid equations iteratively, but assuming Jeans escape at the exobase, $K n=1$. Having achieved a converged solution in this manner, we used the calculated $n\left(r_{\text {od }}\right)$ and $T\left(r_{\text {od }}\right)$ evaluated at $K n\left(r_{\text {od }}\right)=0.1$ as the starting point for the first DSMC iteration. When the solar minimum heating is included the Jeans parameter at the lower boundary for the kinetic model for the converged result was $\lambda\left(r_{\mathrm{od}}\right) \sim 9$. The SHE result taken from Strobel (2008a) is obtained by solving Eqs. (1b) and (1c) using assumed values of $\varphi$ and $(d T / d r)_{0}$, to find a solution with $n, T \rightarrow 0$ and zero total energy flux as $r \rightarrow \infty$.
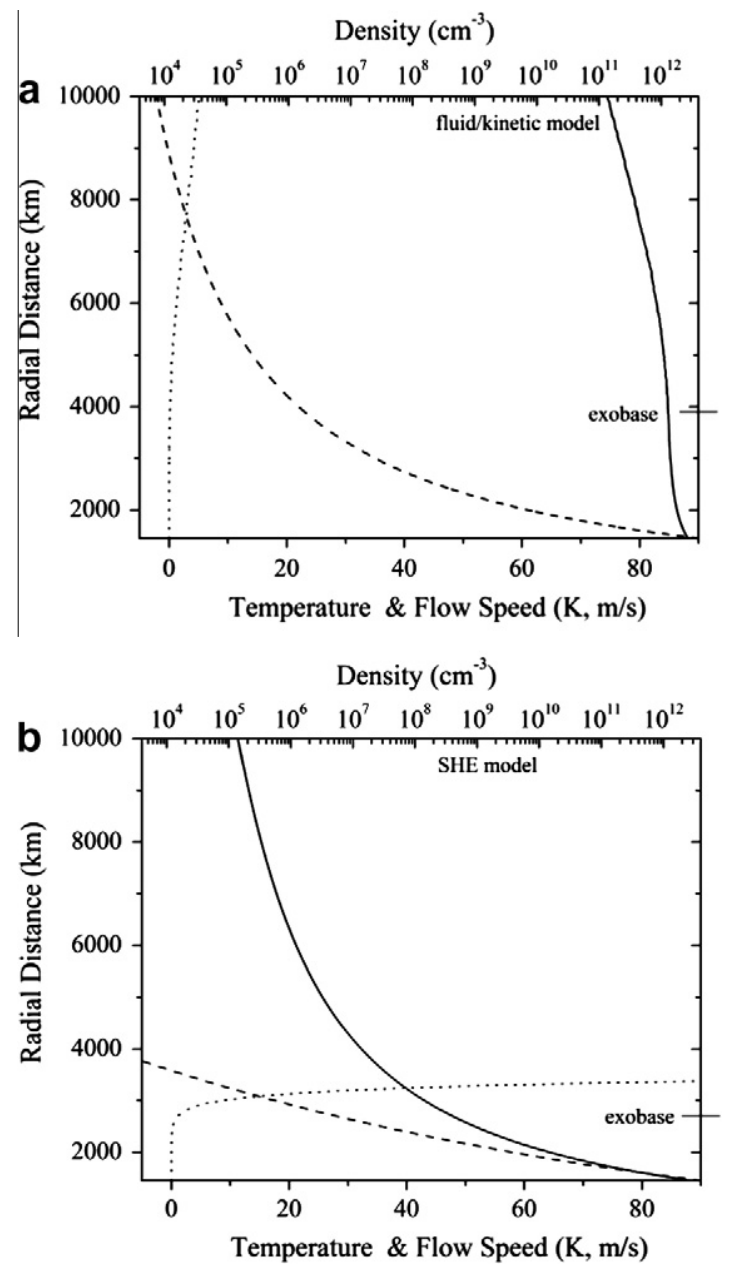

Fig. 3. $n\left(\mathrm{~cm}^{-3}\right)$ (top axis), $T(\mathrm{~K})$ and $u(\mathrm{~m} / \mathrm{s})$ (bottom axis) versus radial distance: Comparison of fluid/kinetic (a) $n$ (dashed curves), $T$ (solid curves) and $u$ (dotted curves) to SHE model results (b) from Strobel (2008a) for no heating above $r_{0}$ $\left(\beta_{0}=0\right)$. The exobase distance is indicated by the solid curve on right axis: $3900 \mathrm{~km}$ fluid/kinetic model and $2700 \mathrm{~km}$ SHE model. The New Horizons spacecraft distance of closest approach to Pluto will be $10,000 \mathrm{~km}$.
As seen in Table 2, the resulting escape rate for the solar minimum case was $1.2 \times 10^{27} \mathrm{~s}^{-1}$ with $r_{x} \sim 6200 \mathrm{~km}, n_{x} \sim 7 \times 10^{5} \mathrm{~cm}^{-3}$, $T_{x} \sim 87 \mathrm{~K}$ and $\lambda_{x} \sim 5$. Although, the escape rate is fortuitously close to the SHE result, $1.8 \times 10^{27} \mathrm{~s}^{-1}$, the structure of the exobase region for the SHE model is very different: $r_{x} \sim 3530 \mathrm{~km}, n_{x} \sim 5.3 \times 10^{6} \mathrm{~cm}^{-3}$, $T_{x} \sim 65 \mathrm{~K}$ and $\lambda_{x} \sim 13$. Therefore, although the SHE escape rate was suggested to be $>10^{3} \times \varphi_{\mathrm{J}}$, based on the temperature and density at the exobase obtained here the escape rate is $2.0 \times \varphi_{\mathrm{J}}$ and the energy flux rate is $1.7 \times\langle E \varphi\rangle_{\mathrm{J}}$. The size of this enhancement to the Jeans rate is similar to that found earlier (Tucker and Johnson, 2009). Ignoring the effect on Charon, this rate is also 84\% of the energylimited escape rate, $\left(4 \pi r_{0}^{2} \beta_{0}\right) /\left(\lambda_{0} k T_{0}\right)$, often used in exoplanet studies (Lammer et al., 2009).

\section{Effect of Charon on escape}

We examine here whether or not these results have implications for Charon, which has $1 / 2$ the diameter and 1/10 mass of Pluto. At an orbital distance of $19,500 \mathrm{~km}$ the Hill sphere radius about Charon is at a radial distance of $12,700 \mathrm{~km}$ from Pluto, where the atmospheric density is $\sim 10^{5} \mathrm{~cm}^{-3}$ for the solar minimum case, Fig. 4a. Ignoring here any tidal effect on Pluto's lower atmosphere we estimated whether the effect of Charon's gravity on the molecular trajectories
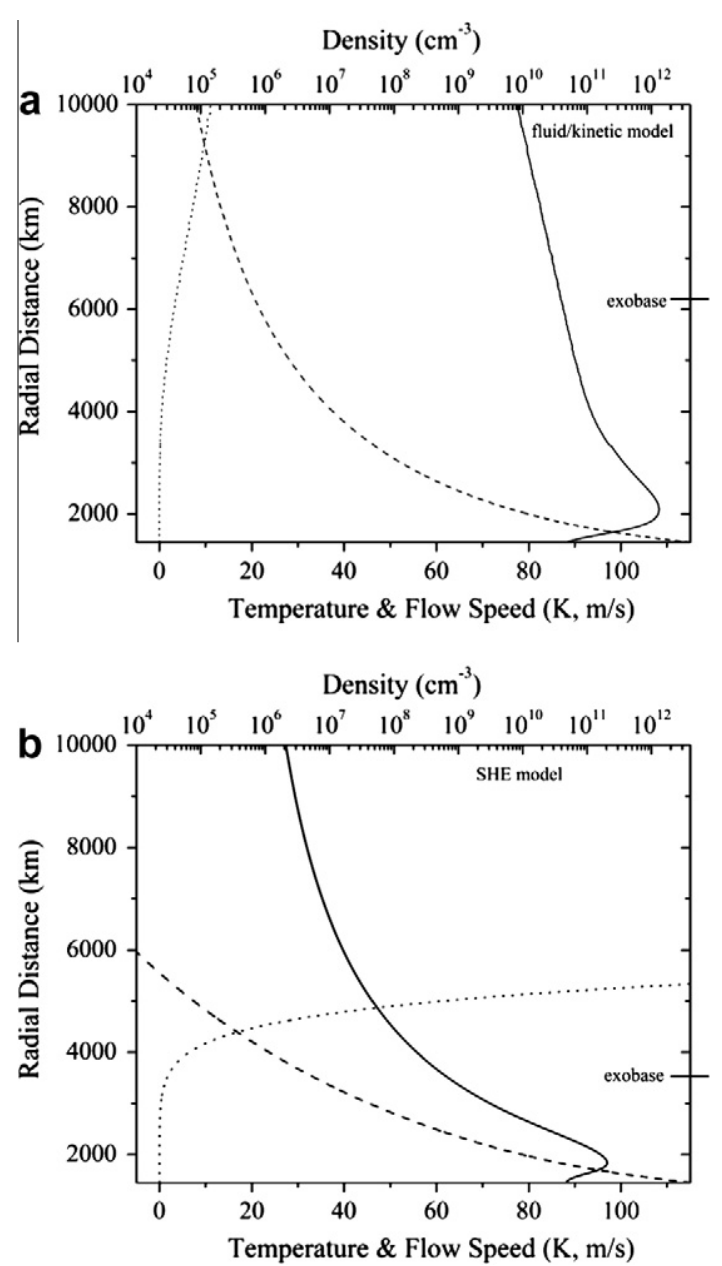

Fig. 4. $n\left(\mathrm{~cm}^{-3}\right)$ (top axis), $T(\mathrm{~K})$ and $u(\mathrm{~m} / \mathrm{s})$ (bottom axis) versus radial distance: Comparison of fluid/kinetic (a) $n$ (dashed curves), $T$ (solid curves) and $u$ (dotted curves) to SHE model results (b) from Strobel (2008a) for solar minimum heating conditions above $r_{0}$. The exobase distance is indicated by the solid curve on right axis: $6200 \mathrm{~km}$ fluid/kinetic model and $3530 \mathrm{~km}$ SHE model. The New Horizons spacecraft distance of closest approach to Pluto will be $10,000 \mathrm{~km}$. 

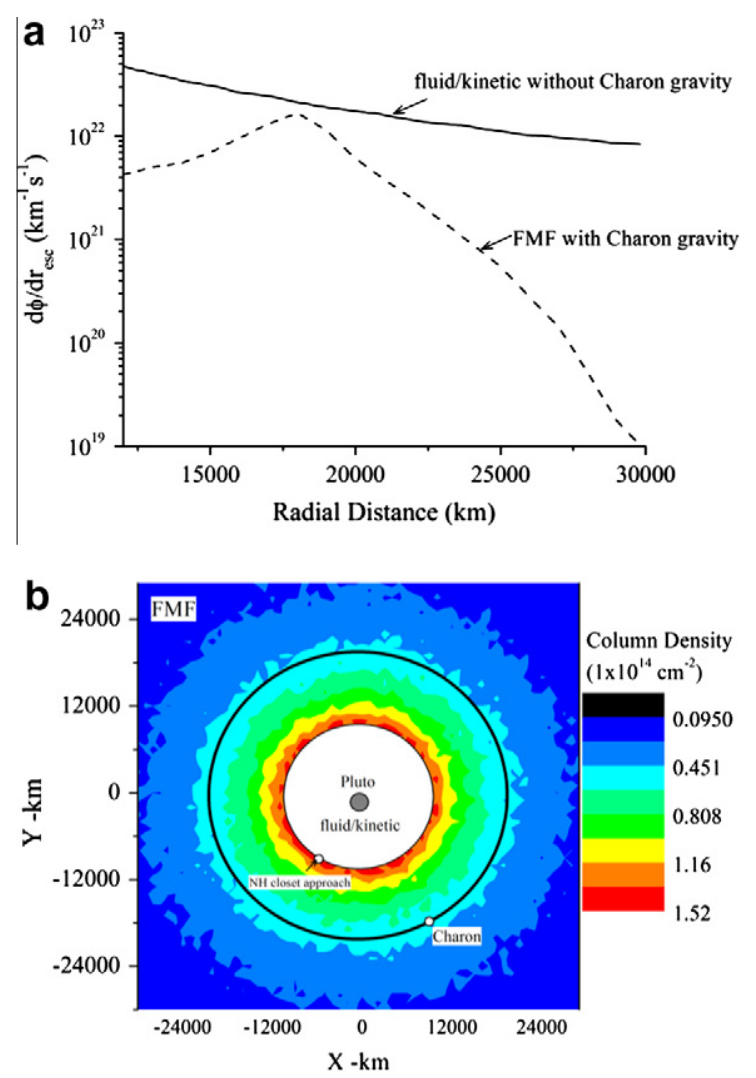

Fig. 5. (a) Production of escaping molecules $d \varphi / d r_{\mathrm{esc}}\left(\mathrm{km}^{-1} \mathrm{~s}^{-1}\right)$ versus $r$. DSMC results for solar minimum heating conditions without including Charon's gravitation influence (solid curve), as in Fig. 2b, compared to the fluid/kinetic result coupled to a FMF model that includes Charon's gravitational effect (dashed curve). (b) Contour plot of column density in the $z$ plane Charon's northern and southern hemispheres in FMF simulations including Charon's gravitational influence: black curve represents Charon's orbit. Particles are emitted radially with corresponding speeds according to the local distribution at $10,000 \mathrm{~km}$. The New Horizons $(\mathrm{NH})$ spacecraft distance of closest approach to Pluto will be $10,000 \mathrm{~km}$.

would significantly affect the escape rate. Therefore, we used the DSMC results for solar minimum conditions to perform free molecular flow (FMF) simulations in which molecules move under the influence of gravity from both Pluto and Charon but without intermolecular collisions. Charon is assumed to have a circular orbit about Pluto and the molecular trajectories are tracked in a 3D region from $10,000 \mathrm{~km}$ to $30,000 \mathrm{~km}$ about Pluto. The FMF simulation is begun by emitting molecules at $r=10,000 \mathrm{~km}$ with radial velocities obtained from the fluid/kinetic model for solar minimum heating conditions. Escaping molecules are either emitted initially with a speed above the escape speed or they gain an escape speed under the gravitational influence of Charon. After several Charon orbits we obtained a steady-state morphology of the gas density in the Pluto-Charon system and the integrated escape rate produced versus radial distance, as shown in Fig. 5a and b.

For solar minimum heating conditions we estimated that molecules from Pluto's atmosphere impinge upon Charon at a rate of $10^{25} \mathrm{~s}^{-1}$. With Charon's surface temperature of $\sim 50 \mathrm{~K}$ this would be equivalent to the deposition of a monolayer of molecules over 4 Charon orbits or $8 \times 10^{-3} \mu \mathrm{m} / \mathrm{yr}$. We also found that Charon has only a small effect on the escape rate from Pluto's atmosphere. Charon does not trap many molecules from Pluto's expanded atmosphere, but rather perturbs the molecules trajectories accelerating them to or decelerating them from escaping the system. The above conclusions were determined by performing FMF simulations with and without the gravitational influence of Charon in which we found that the escape rate decreased by $3 \%$ in simula- tions that included Charon. This is opposite to the change in the escape rate that would have occurred had we used the energy to reach the Hill sphere of Charon as the escape criterion in the fluid/kinetic simulations. A contour plot of the averaged total column densities over the north and south hemispheres when including Charon's gravitational influence is shown in Fig. 5b. The relevance of Charon is likely more significant at solar maximum heating conditions and close to perihelion.

\section{Conclusions}

Hydrodynamic models have often been applied to atmospheres in the Solar System and to exoplanet atmospheres in order to estimate escape and the concomitant adiabatic cooling of the upper atmosphere (e.g., McNutt, 1989; Krasnopolsky, 1999; Tian and Toon, 2005; Strobel, 2008a,b; Yelle, 2004; Tian, 2009; Murray-Clay et al., 2009). Unless the Jeans parameter is $<2$ well below the exobase $(K n \ll 1)$, this procedure can give incorrect atmospheric properties as compared to the fluid/kinetic combined approach described here. The difficulties with using a continuum model of thermal escape are twofold; how to, without prior knowledge, define density, temperature and energy flow at infinity, and how to properly define thermal conduction in the exosphere. The Fourier heat flux used to solve Eq. (2b) becomes invalid near the exobase as discussed earlier (e.g., Johnson, 2010; Volkov et al., 2011a,b). Here we use a combined fluid/kinetic model that explicitly incorporates how heat conduction powers escape without requiring any assumptions about the macroscopic properties of the atmosphere at infinity. The hydrodynamic equations are solved below the exobase, and the kinetic model is continued above where the flow is essentially non-equilibrium. Such a procedure is relevant not only to Pluto but to the evolution of atmospheres on terrestrial bodies including recently discovered hot, rocky exoplanet atmospheres.

For over a few decades, hydrodynamic models have been used to conclude that Pluto's atmosphere is lost by a process called slow hydrodynamic escape (e.g., Krasnopolsky, 1999; McNutt, 1989; Strobel, 2008a,b). We reconsidered escape from Pluto using the fluid/kinetic model and found that for the two cases considered, thermally-driven escape occurs at a rate within a factor of two of the Jeans rate for the temperature determined in the combined model. That is, for a lower boundary, $r_{0}$, in Pluto's atmosphere where $\lambda\left(r_{0}\right) \sim 23$ and $K n\left(r_{0}\right) \sim 10^{-6}$, and with all of the heat deposited below $r_{0}$ (i.e., $\beta_{0}=0$ ), we obtain an escape rate $\varphi \sim 4.8 \times 10^{25} \mathrm{~N}_{2} \mathrm{~s}^{-1}$. For the derived exobase temperature, $T_{x}=85 \mathrm{~K}$, this is $\sim 1.6$ times the Jeans rate $\left(\varphi_{\mathrm{J}} \sim 3.0 \times 10^{25} \mathrm{~N}_{2} \mathrm{~s}^{-1}\right)$ and 1.4 times the Jeans energy flux $\left(\langle E \varphi\rangle_{\mathrm{J}} \sim 7.40 \times 10^{11} \mathrm{ergs} \mathrm{s}^{-1}\right)$. Furthermore we find that each escaping molecule carries off an energy $\sim 2 k T_{0}$ as seen in Table 2 , and not 0 as assumed in the SHE model. It is interesting to note that for the same lower boundary conditions, if one assumed the atmosphere was hydrostatic, the Jeans rate would be $\varphi_{\mathrm{J}} \sim 5.6 \times$ $10^{25} \mathrm{~N}_{2} \mathrm{~s}^{-1}$ and $\langle E \varphi\rangle_{\mathrm{J}} \sim 1.4 \times 10^{12} \mathrm{ergs} \mathrm{s}^{-1}$. Therefore, these simulations indicate escape is similar in nature to Jeans escape, but to get the correct exobase temperature and density needed to make a Jeans estimate, a kinetic model should be applied in the non-equilibrium region of the atmosphere.

We also simulated Pluto's atmosphere for solar minimum conditions above $r_{0}$. For the same density and temperature at $r_{0}$, with a similar solar minimum heating rate to that used in Strobel (2008a), we obtain $\varphi \sim 1.2 \times 10^{27} \mathrm{~N}_{2} \mathrm{~s}^{-1}$. At the derived $T_{x}=87 \mathrm{~K}$ from the model this is $\sim 2.0$ times the Jeans rate $\left(\varphi_{\mathrm{J}} \sim 6.0 \times 10^{26} \mathrm{~N}_{2} \mathrm{~s}^{-1}\right)$ and $\sim 1.7$ times the Jeans energy flux $\left(\langle E \varphi\rangle_{\mathrm{J}} \sim 1.6 \times 10^{13}\right.$ ergs s$\left.^{-1}\right)$. As seen in Table 2 , the escape rate for solar minimum conditions is fortuitously close to that obtained in Strobel (2008a) of $1.8 \times 10^{27} \mathrm{~N}_{2} \mathrm{~s}^{-1}$, but the total energy flux into the lower boundary leads to a very different atmospheric structure in the exobase 
region. Although this energy flux is a small fraction of the energy flux added above $r_{0}$ due to solar heating, $\langle E \varphi\rangle_{r_{0}} /\left(4 \pi r_{0}^{2} \beta_{0}\right)=6.8 \times 10^{-2}$, it influences density and temperature gradients below the heating peak where $\beta(r) \rightarrow 0$ as $r \rightarrow r_{0}$. Furthermore the flow fields are radically different. This may be illustrated by comparing the relative magnitudes of the static, $p$, and dynamic, $1 / 2 m u^{2}$, pressures of each model. For the radial distance examined, $1450-10,000 \mathrm{~km}$, we find the total pressure profile of the fluid/kinetic model monotonically decreases and is dominated by the static pressure. On the other hand, the SHE model experiences a minimum in the dynamic pressure at $5700 \mathrm{~km}$ with the region below dominated by the static pressure and the region above dominated by the dynamic pressure. The sum of the dynamic and static pressures in the SHE model also exceeds that of the fluid/kinetic model above $8200 \mathrm{~km}$, suggesting that despite their similar flux rates the two models may be observationally distinguishable in their determination of Pluto's interaction with the solar wind.

We show here that starting at a small $K n\left(r_{0}\right)$ in Pluto's atmosphere, a combined fluid/kinetic model can lead to reliable energy and molecule escape rates both for no heating and solar minimum heating conditions in the region above $1450 \mathrm{~km}$. It is also clear from the fluid and DSMC results in the overlap region, that for this range of Jeans parameters a fluid model can obtain accurate temperatures densities and gas velocities with similar heat fluxes up to the exobase. But this is the case only if the $\varphi$ and $\langle E \varphi\rangle_{r_{0}}$ used are equal to that obtained from a kinetic simulation of the exobase region. In fact, the total energy flux through the system cannot be determined independently for finite $K n\left(r_{0}\right)$ using a fluid calculation, because it depends on the flow in the non-equilibrium region of the exosphere. Numerical methods have been used to solve the hydrodynamic model using a Jeans type escape and energy flux at or near the exobase (Chamberlain, 1961; Yelle, 2004; Gruzinov, 2011). However, these models require assumed values for $n$ and $T$ at the upper boundary.

We have treated Pluto's atmosphere using a single species, $\mathrm{N}_{2}$, throughout the simulation region and have found an enhanced Jeans rate like that found earlier (Tucker and Johnson, 2009). Although minor species, with very different masses, will separate from the $\mathrm{N}_{2}$ profile in the region of escape (e.g., Tucker and Johnson, 2009), CO should roughly track the $\mathrm{N}_{2}$ profile described here. Since the solar activity during the observations of Greaves et al. (2011) in 2009/2010 was close to that used for our assumed solar minimum conditions, the discovery of CO at altitudes $\sim 4500 \mathrm{~km}$ might not be surprising based on the atmospheric structure found here and does not require understanding the interaction of the extended atmosphere with the solar wind. Based on an assumed mixing ratio of $\sim 0.05 \%$ the $\mathrm{CO}$ tangential column density at $4500 \mathrm{~km}$ would be $\sim 6 \times 10^{11} \mathrm{CO} \mathrm{cm}^{-2}$, but the temperature at this altitude is $91 \mathrm{~K}$ as opposed to $50 \mathrm{~K}$ suggested by the observations.

Solar maximum conditions are expected to occur in 2013, so that the New Horizon encounter with Pluto and Charon in 2015 will occur somewhere between solar maximum and minimum conditions. At a distance from the Sun of $33 \mathrm{AU}$ and assuming the same heating efficiency and cooling process, this results in $\beta_{0} \sim 1.7$ times that used here $\left(\sim 2.5 \times 10^{-3} \mathrm{erg} \mathrm{cm}^{-2} \mathrm{~s}^{-1}\right)$. Therefore, accurate simulations of the atmospheric density at the encounter distance $10,000 \mathrm{~km}$ from Pluto, and the atmospheric structure and the escape rates expected during the encounter will require the use of a fluid/kinetic model such as that described here. Such calculations are in progress for a multispecies atmosphere.

\section{Acknowledgments}

We thank D. Strobel for the solar minimum heating rates, L. Young for information on Pluto's atmosphere, and R. Yelle and A.
Gruzinov for helpful comments on atmospheric escape. We also note the loss of two pioneers in the field whose papers we heavily relied upon, D. Hunten and J. Elliot. This research was supported by the NASA Planetary Atmospheres Program and the NSF Astronomy Program.

\section{References}

Bird, G.A., 1994. Molecular Gas Dynamics and the Direct Simulation of Gas Flows. Clarendon Press, Oxford, New York.

Burden, R.L., Faires, J.D., 2005. Numerical Analysis, eighth ed. Thomson Brooks/Cole, Belmont, CA, USA, pp. 285, 343.

Chamberlain, J.W., 1960. Interplanetary gas. II. Expansion of a model of the solar corona. Astrophys. J. 131, 47-56.

Chamberlain, J.W., 1961. Interplanetary gas. III. Hydrodynamic model of the corona. Astrophys. J. 133, 675-687.

Chamberlain, J.W., Hunten, D., 1987. Theory of Planetary Atmospheres. Academic Press, New York.

Chapman, S., Cowling, T.G., 1970. The Mathematical Theory of Non-uniform Gases, third ed. Cambridge University Press, New York (Chapter 10).

Elliot, J.L. et al., 2007. Changes in Pluto's atmosphere: 1988-2006. Astrophys. J. 134, $1-13$

Greaves, J.S., Helling, Ch., Friberg, P., 2011. Discovery of carbon monoxide in the upper atmosphere of Pluto. Mon. Not. R. Astron. Soc. 000, 1-6.

Gruzinov, A., 2011. The Rate of Thermal Atmospheric Escape. arXiv:1101.1103v1 [astro-ph.EP] 5 January 2011.

Hubbard, W.B., Yelle, R.V., Lunine, J.I., 1990. Nonisothermal Pluto atmosphere models. Icarus 84, 1-11.

Hunten, D.M., 1973. The escape of light gases from planetary atmospheres. J. Atmos. Sci. 30, 1481-1494.

Hunten, D.M., Watson, A.J., 1982. Stability of Pluto's atmosphere. Icarus 51, 665667.

Jeans, J.H., 1916. The Dynamical Theory of Gases: The Outer Atmosphere. Cambridge University Press, pp. 351-363.

Johnson, R.E., 2010. Thermally driven atmospheric escape. Astrophys. J. 716, 15731578.

Krasnopolsky, V.A., 1999. Hydrodynamic flow of $\mathrm{N}_{2}$ from Pluto. J. Geophys. Res. 104 5955-5962.

Lammer, H. et al., 2009. Determining the mass loss limit for close-in exoplanets: what can we learn from transit observations? Astron. Astrophys. 506, 399410.

Marconi, M.L., Dagum, L., Smyth, W.H., 1996. Hybrid fluid/kinetic approach to planetary atmospheres: An example of an intermediate mass body. Astrophys. J. 469, 393-401.

McNutt, R.L., 1989. Models of Pluto's upper atmosphere. Geophys. Res. Lett. 16, 1225-1228.

Murray-Clay, R.A., Chiang, E.I., Murray, N., 2009. Atmospheric escape from hot Jupiters. Astrophys. J. 693, 23-42.

Öpik, E.J., 1963. Selective escape of gases. Geophys. J. Roy. Astron. Soc. 7, 490-509.

Parker, E.N., 1958. Dynamics of the interplanetary gas and magnetic fields. Astrophys. J. 128, 664-676.

Parker, E.N., 1964a. Dynamical properties of stellar coronas and stellar winds. I. Integration of the momentum equation. Am. Astron. Soc. 139, 72-92.

Parker, E.N., 1964b. Dynamical properties of stellar coronas and stellar winds. II. Integration of the heat-flow equation. Am. Astron. Soc. 139, 93-122.

Strobel, D.F., 2008a. $\mathrm{N}_{2}$ escape rates from Pluto's atmosphere. Icarus 193, 612619.

Strobel, D.F., 2008b. Titan's hydrodynamically escaping atmosphere. Icarus 193, 588-594.

Tian, F., 2009. Thermal escape from super Earth atmosphere in the habitable zone of M stars. Astrophys. J. 703, 905-909.

Tian, F., Toon, O.B., 2005. Hydrodynamic escape of nitrogen from Pluto. J. Geophys. Res., L18201-L18205.

Trafton, L., 1980. Does Pluto have a substantial atmosphere? Icarus 44, 53-61.

Tucker, O.J., Johnson, R.E., 2009. Thermally driven atmospheric escape: Monte Carlo simulations for Titan's atmosphere. Planet. Space Sci. 57, 1889-1894.

Tucker, O.J., Erwin, J.T., Volkov, A.N., Cassidy, T.A., Johnson, R.E., 2011. Escape from Pluto's atmosphere: Fluid/DSMC hybrid simulation. In: Proc. 27th International Symposium on Rarefied Gas Dynamics, Pacific Grove, USA, 2010, AIP Conf. Proc. 1333, pp. 1145-1150.

Volkov, A.N., Johnson, R.E., Tucker, O.J., Erwin, J.T., 2011a. Thermally-driven atmospheric escape: Transition from hydrodynamic to Jeans escape. Astrophys. J. 729, L24, 1-5.

Volkov, A.N., Tucker, O.J., Erwin, J.T., Johnson, R.E., 2011b. Kinetic simulations of thermal escape from a single component atmosphere. Phys. Fluid 23, 066601 $1-16$.

Watson, A.J., Donahue, T.M., Walker, J.C.G., 1981. The dynamics of a rapidly escaping atmosphere: Applications to the evolution of Earth and Venus. Icarus 48, 150166.

Yelle, R.V., 2004. Aeronomy of extra-solar giant planets at small orbital distances. Icarus 170, 167-179.

Young, E.F. et al., 2008. Vertical structure in Pluto's atmosphere from the 2006 June 12 stellar occultation. Astrophys. J. 136, 1757-1769. 


\title{
MOLECULAR-KINETIC SIMULATIONS OF ESCAPE FROM THE EX-PLANET AND EXOPLANETS: CRITERION FOR TRANSONIC FLOW
}

\author{
Robert E. Johnson ${ }^{1,2}$, Alexey N. Volkov ${ }^{1}$, And Justin T. Erwin ${ }^{1}$ \\ ${ }^{1}$ Engineering Physics, University of Virginia, Charlottesville, VA 22904-4745, USA \\ 2 Physics Department, New York University, NY 10003-6621, USA \\ Received 2013 March 1; accepted 2013 March 19; published 2013 April 12
}

\begin{abstract}
The equations of gas dynamics are extensively used to describe atmospheric loss from solar system bodies and exoplanets even though the boundary conditions at infinity are not uniquely defined. Using molecular-kinetic simulations that correctly treat the transition from the continuum to the rarefied region, we confirm that the energylimited escape approximation is valid when adiabatic expansion is the dominant cooling process. However, this does not imply that the outflow goes sonic. Rather large escape rates and concomitant adiabatic cooling can produce atmospheres with subsonic flow that are highly extended. Since this affects the heating rate of the upper atmosphere and the interaction with external fields and plasmas, we give a criterion for estimating when the outflow goes transonic in the continuum region. This is applied to early terrestrial atmospheres, exoplanet atmospheres, and the atmosphere of the ex-planet, Pluto, all of which have large escape rates.
\end{abstract}

Key words: hydrodynamics - molecular processes - planets and satellites: atmospheres

Online-only material: color figures

\section{INTRODUCTION}

Rapid atmospheric escape is often described as a gas that goes sonic, sometimes called blow-off (Hunten 1982), a process that accounts for certain isotope ratios on terrestrial planets. Transonic models have also been used to describe rapid escape from exoplanets (e.g., Murray-Clay et al. 2009) and from Pluto (e.g., Strobel 2008). However, we recently showed that this model for Pluto gave an incorrect upper atmospheric structure (Tucker et al. 2012; Erwin et al. 2013).

In simulating rapid escape using continuum gas dynamics, the Jeans expressions at the exobase (Chamberlain \& Hunten 1987) have been applied for the uncertain boundary conditions at infinity (e.g., Tian et al. 2008). More often, a sonic point is assumed to occur at some altitude, above which the density and temperature dependence can be simply characterized (Parker 1964a, 1964b). The so-called energy-limited escape rate, extensively applied to exoplanet atmospheres (e.g., Lammer et al. 2009), is often assumed to imply that sonic boundary conditions are applicable (e.g., Erkaev et al. 2012). Here we use molecularkinetic simulations to show that is not the case.

We briefly review the continuum and molecular-kinetic models, and then present results of our simulations. These test the applicability of the energy-limited escape rate and our proposed criterion for determining whether sonic or kinetic upper boundary conditions are applicable. The results are applied to escape from Pluto, early terrestrial planets, and exoplanet atmospheres.

\section{MODELS}

We describe escape from a one-dimensional (1D), steadystate, single-component atmosphere as illustrative, leaving out thermal transport by horizontal flow. For radial distance $r$, flow speed $u$, number density $n$, temperature $T$, pressure $p=n k T$, and escape rate $\Phi=4 \pi r^{2} n u=$ constant, the momentum and energy equations are often used ignoring viscosity:

$$
n \frac{d}{d r}\left(m u^{2} / 2-U\right)=-\frac{d p}{d r}
$$

$$
\frac{d}{d r}\left[\Phi\left(m u^{2} / 2+C_{p} k T-U\right)-4 \pi r^{2} \kappa \frac{d T}{d r}\right]=4 \pi r^{2} n q_{a}(r) .
$$

Here $k$ is the Boltzmann constant, $\kappa=\kappa(T)$ is the thermal conductivity, $C_{P}$ is the heat capacity at constant pressure, $m$ is the molecular mass, $U=U(r)=G M m / r$ is the gravitational energy ( $G$ is the gravitational constant and $M$ is the planet's mass), and $q_{a}(r)$ is the net heating rate per molecule produced by incident photons or plasma particles, in which we include radiative cooling. Knowing the density, $n_{0}$, and temperature, $T_{0}$, at a lower boundary, $r=r_{0}$, a unique solution requires two other conditions, typically at the upper boundary. The gravitational energy is characterized by the Jeans parameter, $\lambda(r)=U / k T$, and the rarefaction by the Knudsen number, $K n(r)=l_{c} / H$, the ratio of the mean free path of gas molecules, $l_{c}$, to the scale height, $H=-n /(d n / d r)$. For an escaping gas at large distances from the source, where free molecular flow occurs, $H \rightarrow r / 2$; in the hydrostatic regime $H \rightarrow r / \lambda(r)$.

The Jeans expressions for the number, $\Phi_{J}$, and thermal, $\langle E \Phi\rangle_{J}$, escape rates have been used as upper boundary conditions for Equations (1) and (2):

$$
\begin{gathered}
\Phi_{J}=4 \pi r_{x}^{2} n_{x} \sqrt{\frac{k T_{x}}{2 \pi m}}\left(1+\lambda_{x}\right) \exp \left(-\lambda_{x}\right) \\
\langle E \Phi\rangle_{J}=k T_{x} \Phi_{J}\left(\frac{1}{1+\lambda_{x}}+C_{p}-\frac{3}{2}\right)
\end{gathered}
$$

The subscript " $x$ " indicates quantities evaluated at the nominal exobase, $r=r_{x}$, where $K n\left(r_{x}\right) \approx 1$, often assumed to be the upper boundary of the continuum region. When the upper atmosphere heating rate is large, the equations are more often solved through a sonic point, $r=r_{*}$, where $u_{*}=c$ (with $c=\sqrt{\gamma k T / m}$ being the sound speed; $\gamma=C_{p} / C_{V}, C_{V}$ being the heat capacity at constant volume). For $r \gg r_{*}$ then $n$ and $T$ decay as power laws (Parker 1964a, 1964b). Unfortunately, those continuum solutions for which Jeans escape is applicable and those for which a sonic point is reached in the continuum 
region do not simply track from one to the other as the heating rate increases.

Kinetic models can simulate both continuum $\left(l_{c} \ll H\right)$ and non-continuum (transitional, $l_{c} \sim H$, and free molecular, $\left.l_{c} \gg H\right)$ gas flows and can therefore describe the change from Jeans-like to transonic escape. Since such simulations track particles in the potential well of the body (or bodies) of interest, escape is a natural outcome. We numerically implement a kinetic description of rarefied gas flow in an upper atmosphere based on the Boltzmann kinetic equation using the direct simulation Monte Carlo (DSMC) method (Bird 1994). In this method, the gas flow is represented by a large set of representative atoms or molecules that are tracked subject to binary collisions and gravity (Volkov 2011a, 2011b). Heating of the atmosphere is implemented by scaling the thermal velocities of the representative molecules according to the local energy deposition rate.

The lower boundary of the simulation region, $r=r_{0}$, is below the depth at which the UV/EUV or plasma energy deposition occurs and $K n_{0} \ll 1$. Because the density drops rapidly with increasing $r$, but escape occurs at large $r$ where the density is low, DSMC simulations starting at small $K n_{0}$ can require an enormous number of particles to accurately describe escape. Therefore, we also use a hybrid continuum/ kinetic model (Tucker et al. 2012) in which Equations (1) and (2) are solved at $K n<\sim 0.1-0.01$, where the gas is collisionally dominated, and the velocity and internal energy distributions are reasonably well represented by Boltzmann distributions, and then iteratively couple it to a DSMC simulation in the rarefied region.

\section{HEATING}

Parker (1964a, 1964b) used Equations (1) and (2) to describe escape when the dominant heat source is internal, as it is for expansion of the solar corona: i.e., $q_{a}(r)=0$ for $r>r_{0}$. This model was subsequently applied to planetary atmospheres primarily heated at $r<r_{0}$. For Jeans parameters at $r=r_{0}$ as large as $\lambda_{0} \sim 40$, such models were assumed to produce a transonic expansion, often referred to as "slow hydrodynamic escape" (e.g., Strobel 2008). Although rapid escape can occur for relatively large $\lambda_{0}$ and $K n_{0} \ll 1$, for $\lambda_{0}>\sim\left(C_{p}+\gamma / 2\right)$ the gas does not go sonic in the collision-dominated region and the escape rate is a factor of a few larger than the Jeans rate (Volkov et al. 2011a, 2011b). Ignoring the thermal conductivity in Equation (2), this corresponds to when the enthalpy of fluid particles becomes sufficient for a transonic, isentropic expansion starting at $r_{0}$. For smaller $\lambda_{0}$ a non-equilibrium region, a Knudsen layer, forms above $r=r_{0}$ and there is a steep transition with decreasing $\lambda_{0}$ to supersonic escape at $\lambda_{0} \sim 2.1$ and $\sim 2.8-3.5$ for monatomic and diatomic gases, respectively (Volkov \& Johnson 2013).

In an upper atmosphere heated by short wavelength radiation, or by incident plasma particles, escape is driven by the energy absorbed. Energy absorbed below $K n \sim 0.1$ is typically converted to heat using an efficiency, $\varepsilon$, that depends on the radiation type and atmospheric composition. The heating rate is either directly calculated or a value of $\varepsilon$ is estimated: often a constant $(\sim 0.15-0.4)$ up to the exobase where it goes to zero. Typically the gas-dynamic equations for exoplanets or early terrestrial atmospheres are then solved with Jeans-like or sonic upper boundary conditions. As discussed below, we used a hybrid continuum/DSMC model to describe escape from Pluto heated by the solar UV/EUV and a DSMC model to describe escape from an atmosphere in which the heating is assumed to occur in a narrow layer.

\section{ENERGY-LIMITED ESCAPE}

Because thermal conduction in the upper atmosphere is inefficient, adiabatic cooling by escape or horizontal transport often dominates (e.g., Erwin et al. 2013). For a globally averaged heating rate and adiabatic cooling, the integration of Equation (2) gives a rough upper bound to the escape rate, $\Phi_{E L}$ (e.g., Lammer et al. 2009):

$$
\Phi_{E L} \approx Q_{\text {net }} /\left.\left(U-C_{p} k T-m u^{2} / 2\right)\right|_{r=r_{0}} .
$$

Here $r_{0}$ is below the heated region, above which adiabatic cooling dominates; $Q_{\text {net }}=4 \pi \int_{r_{0}}^{\infty} r^{2} q_{a}(r) d r$ is the integrated heating+radiative cooling rate. This expression is often referred to as the energy-limited rate, although Watson et al. (1981) discussed a related quantity. For $r_{0}$ deep in the gravitational well and $\lambda_{0} \gg C_{p}$, Equation (4) is often approximated as

$$
\Phi_{E L} \approx Q_{\mathrm{net}} / U\left(r_{0}\right)
$$

Assuming only a very small fraction of $Q_{\text {net }}$ is deposited at $K n>\sim 0.1$, so non-thermal escape processes (Johnson et al. 2008) can be ignored, we showed that UV/EUV heating of Pluto's atmosphere resulted in an escape rate very close to that in Equation (5), but the gas did not go sonic below the exobase. Rather, a large expansion of the upper atmosphere occurred (Tucker et al. 2012; Erwin et al. 2013). Similarly, Tian et al. (2008) found that above a heating threshold, the atmosphere rapidly expanded and the escape rate increased with increasing EUV heating, consistent with energy-limited escape, even though the gas remained subsonic. Therefore, the energylimited escape rate is not contingent on the flow going sonic below the exobase. If the heating rate is increased to the point where the atmosphere does go sonic in the continuum region, energy-limited escape can still apply if one accounts for the large $u$, enhanced radiative cooling, recombination in an ionized atmosphere, etc. (e.g., Murray-Clay et al. 2009).

\section{CRITERION FOR TRANSONIC SOLUTIONS}

Since the isentropic approximation and energy-limited escape are applicable to both subsonic and transonic rapid outflows, we use the Mach number, $M a=u / \sqrt{\gamma k T / m}$, and rewrite Equation (5):

$$
Q_{\text {net }} \approx 4 \pi r^{2} n M a \sqrt{\frac{\gamma}{\lambda} \frac{U(r)}{m}} U\left(r_{0}\right) .
$$

Because the boundary conditions for subsonic and transonic solutions differ, it is important to be able to estimate the minimum value of $Q_{\text {net }}$ required to apply sonic boundary conditions, which we will call $Q_{c}$. Assuming the sonic point, $r=r_{*}$, occurs in the continuum region, the flow can be effectively approximated by the isentropic model:

$$
2 m c^{2}\left(r_{*}\right)=U\left(r_{*}\right)+(\gamma-1) \frac{r_{*} q\left(r_{*}\right)}{u\left(r_{*}\right)} .
$$

(e.g., Murray-Clay et al. 2009). For $q\left(r_{*}\right) \approx 0$ Equation (7) reduces to $\lambda_{*} \approx 2 \gamma$ and transonic escape occurs when $M a>1$ 
in Equation (6) giving

$$
Q_{\text {net }}>Q_{c} \approx 4 \pi r_{*}^{2} n_{*} \sqrt{\frac{U\left(r_{*}\right)}{2 m}} U\left(r_{0}\right),
$$

Below we estimate $Q_{c}$ and test it.

We first consider a narrow heating layer located at $r=r_{a}>r_{0}$ with $K n\left(r_{a}\right) \ll 1$, as in Watson et al. (1981) and McNutt (1989), and give an approximate analytic solution to Equations (1) and (2) in the Appendix. For this case, we performed DSMC simulations for a monatomic gas of hard spheres with $\lambda_{0}=10$, $K n_{0}=10^{-3}, r_{a} / r_{0}=1.1$. For $Q_{\text {net }}=0$, we showed earlier that these conditions correspond to enhanced Jeans-like escape with $\Phi \approx 1.6 \Phi_{J}$ (Volkov et al. 2011a, 2011b). Increasing $Q_{\text {net }}$ to find when the outflow goes sonic in the continuum regime, it is seen in Figure 1 that for $Q_{\text {net }} \gg 0$ a non-equilibrium layer forms near $r_{a}$ in which the parallel and perpendicular components of temperature differ. For small $K n\left(r_{a}\right)$ the flow properties in this layer are analogous to those in the Knudsen layer discussed above for heating below $r_{0}$. Near $r_{a}$ the density and temperature change dramatically but the pressure from $r_{0}$ to $\sim r_{a}$ can be estimated from the hydrostatic approximation. When the outflow goes sonic, a pressure drop occurs from $\sim r_{a}$ to $r_{*}$ : $P_{c}=p_{*} / p_{a}$ (Volkov \& Johnson 2013). Since the transition layer is narrow, $r_{*} \approx r_{a}$, we rewrite Equation (8) using values at $r_{0}$ :

$$
Q_{\mathrm{net}}>Q_{c} \approx\langle E \Phi\rangle_{0}\left[2 \gamma \sqrt{\pi \lambda_{0}}\left(\frac{r_{a}}{r_{0}}\right)^{\frac{5}{2}} P_{c} \frac{p_{a}}{p_{0}}\right]
$$

Here $\langle E \Phi\rangle_{0}=4 \pi r_{0}^{2} n_{0} k T_{0} \sqrt{k T_{0} /(2 \pi m)}$ is the upward Maxwellian energy flux of molecules leaving the source at $r=r_{0}$. When the effects of thermal conduction are small relative to adiabatic cooling, then Equation (9) can be derived from the analytic expression in the Appendix. The barometric equation gives $p_{a} / p_{0} \approx \exp \left[\lambda_{0}\left(r_{0} / r_{a}-1\right)\right]$ with $P_{c}$ depending on the number of degrees of freedom: $\sim 0.4$ for a monatomic gas. From Figure 1, the transition to supersonic flow occurs for $0.46<Q_{\text {net }} / Q_{c}<0.67$. Due to the above approximations, Equation (9) overestimates $Q_{c}$ by about a factor of two. It is also seen in Figure 1(c) that $n(r)$ for the subsonic solution increases slowly for $r \gg r_{a}$, resulting in a significantly expanded atmosphere, but $n(r)$ for the transonic solution in Figure 1(e) roughly decreases as a power law consistent with transonic escape.

As important, the escape rate increases dramatically above $Q_{\text {net }}=0$ and becomes close to $\Phi_{E L}$ in Equation (5) in the subsonic regime as seen in Figure 2. Although $\Phi$ does not change significantly in the transition to transonic escape, a steep increase is seen in the thermal+flow energy removed, $\langle E \Phi\rangle$. It is also seen that additional heating primarily increases the average kinetic energy of escaping molecules so that Equation (5) becomes a poor approximation if $Q_{\text {net }} \gg Q_{c}$.

We now consider more realistic heating profiles. In order to apply sonic boundary conditions in Equations (1) and (2), $K n\left(r_{*}\right)$ must be in the continuum region below some maximum, $K n_{m}$ : i.e., $K n\left(r_{*}\right)=l_{c *} / H_{*}<K n_{m}$. Using $H_{*} \sim r_{*} / \lambda_{*}$ and $l_{c *}=1 /\left(c_{c} \sigma_{c} n_{*}\right)$, where $c_{c}$ is determined by the energy dependence of the total collision cross section $\sigma_{c}$ (e.g., $c_{c}=\sqrt{2}$, $\sigma_{c}=\pi d^{2}$ for the gas of hard sphere molecules of diameter $d$ ), then $2 \gamma /\left(c_{c} r_{*} \sigma_{c} n_{*}\right)<K n_{m}$. From Equation (8), we estimate
$Q_{c}$ when $r_{*}$ occurs in the continuum domain:

$$
Q_{\text {net }}>Q_{c} \approx 4 \pi r_{*} \frac{\gamma}{c_{c} \sigma_{c} K n_{m}} \sqrt{\frac{2 U\left(r_{*}\right)}{m}} U\left(r_{0}\right),
$$

where $r_{0}<r_{*}<r_{\mathrm{x}}$. When there is a sharp change in the gas properties, as for the heated layers discussed above, then $K n_{m}<\sim 0.1$ (Volkov \& Johnson 2013). However, if the heat is primarily absorbed over a broad range of $r$ below $r_{x}$, then $K n_{m} \sim 1$ is sufficient.

It is seen in Equation (10) that $Q_{c}$ does not explicitly depend on $T_{0}$, consistent with simulations when $\Phi$ is large. Because $Q_{c}$ depends on the sonic point only via $\left(r_{*}\right)^{1 / 2}$, a rough lower bound can be obtained by replacing $r_{*}$ with the mean energy absorption depth, $r_{a}$ estimated from the absorption cross section, $\sigma_{a}$. More accurately, at threshold the sonic point approaches $r_{x}$ so that $r_{*} \sim r_{a}\left[1+\left(\sigma_{a} / c_{c} \sigma_{c}\right) \lambda_{\text {ave }}\right]$ where $\lambda_{\text {ave }} \sim\left(\lambda_{a}+2 \gamma\right) / 2$ slightly increasing $Q_{c}$. For a close-in exoplanet, tidal heating can be included in $U(r)$ and ion escape can dominate so that $\sigma_{c}$ becomes large due to ion-neutral or ion-ion collisions reducing $Q_{c}$.

For solar minimum, medium, and maximum conditions we simulated Pluto's upper atmosphere at the New Horizons encounter using our hybrid fluid/kinetic model ignoring the interaction with the solar wind and Charon, as well as nonthermal escape (Erwin et al. 2013). For all three cases $\left(Q_{\text {net }}=\right.$ $\left.0.38,0.78, \sim 1.6 \times 10^{8} \mathrm{~W}\right)$ the atmosphere became highly extended, but the flow remained subsonic contrary to all earlier models (e.g., Strobel 2008) with the escape rate close to the energy-limited estimate in Equation (4). It is seen in Figure 3 that at solar medium $r_{x}$ is more than twice that obtained when sonic boundary conditions are applied and, although the escape rate is large, using the Jeans boundary conditions results a much better approximation to the upper atmosphere structure.

For UV/EUV absorption at $r_{a} \sim 1.5$ times Pluto's radius, $r_{p}$, using $K n_{m} \sim 1$ and $r_{*} \sim r_{a} \sim r_{0}$, Equation (10) gives $Q_{c}>\sim 10 \times 10^{8}$. This is well above the largest heating rate $\left(\sim 1.6 \times 10^{8} \mathrm{~W}\right)$ in Erwin et al. (2013). Therefore, Pluto's atmosphere at the New Horizons encounter will be highly extended with an escape rate very close to the energy-limited rate, but the flow in the continuum region will be subsonic.

In order to further test Equation (10), we performed DSMC simulations with a distributed heating model in which, for simplicity, we used Beer's law along the radial direction (MurrayClay et al. 2009): $q_{a}(r)=\varepsilon \sigma_{a} n(r) F_{\mathrm{UV} / \mathrm{EUv}} \exp \left(-\sigma_{a} N(r)\right)$, where $\sigma_{a}, \varepsilon$, and $F_{\mathrm{UV} / \text { EUV }}$ are the absorption cross section, the heating efficiency, and solar energy flux at the upper boundary of our domain, $r=r_{u}$, and $N(r)=\int_{r}^{r_{u}} n(r) d r$, with constant $\varepsilon$ out to $r_{u}$. The transition to a transonic solution with $r_{*}$ in the continuum region of the atmosphere was found to occur at $0.69<Q_{\text {net }} / Q_{c}<1.6$, in agreement with our criterion $Q_{c}$ calculated from Equation (10).

The threshold for transonic flow can be related to the absorbed energy converted to heat, $Q_{a}$, by accounting for the non-adiabatic cooling processes, $Q_{\text {cool }}: Q_{\text {net }} \sim Q_{a}-Q_{\text {cool }}$ (Erwin et al. 2013, Figure 4(b)). If $Q_{\text {cool }}$ is small, writing $Q_{a} \sim \varepsilon \pi r_{a}^{2} F_{\mathrm{UV} / \mathrm{EUV}}$, then $\Phi_{E L}$ in Equation (5) can be roughly scaled by the luminosity if the solutions are subsonic or do not significantly exceed $Q_{c}$. Because the atmospheric expansion affects $r_{a}$, iterative solutions can improve estimates of $Q_{a}$. Ignoring this, we note that for an early Earth-like upper atmosphere dominated by $\mathrm{N}$ or $\mathrm{O}$, a solar EUV flux more than 100 times the present would be required for the escaping gas to 

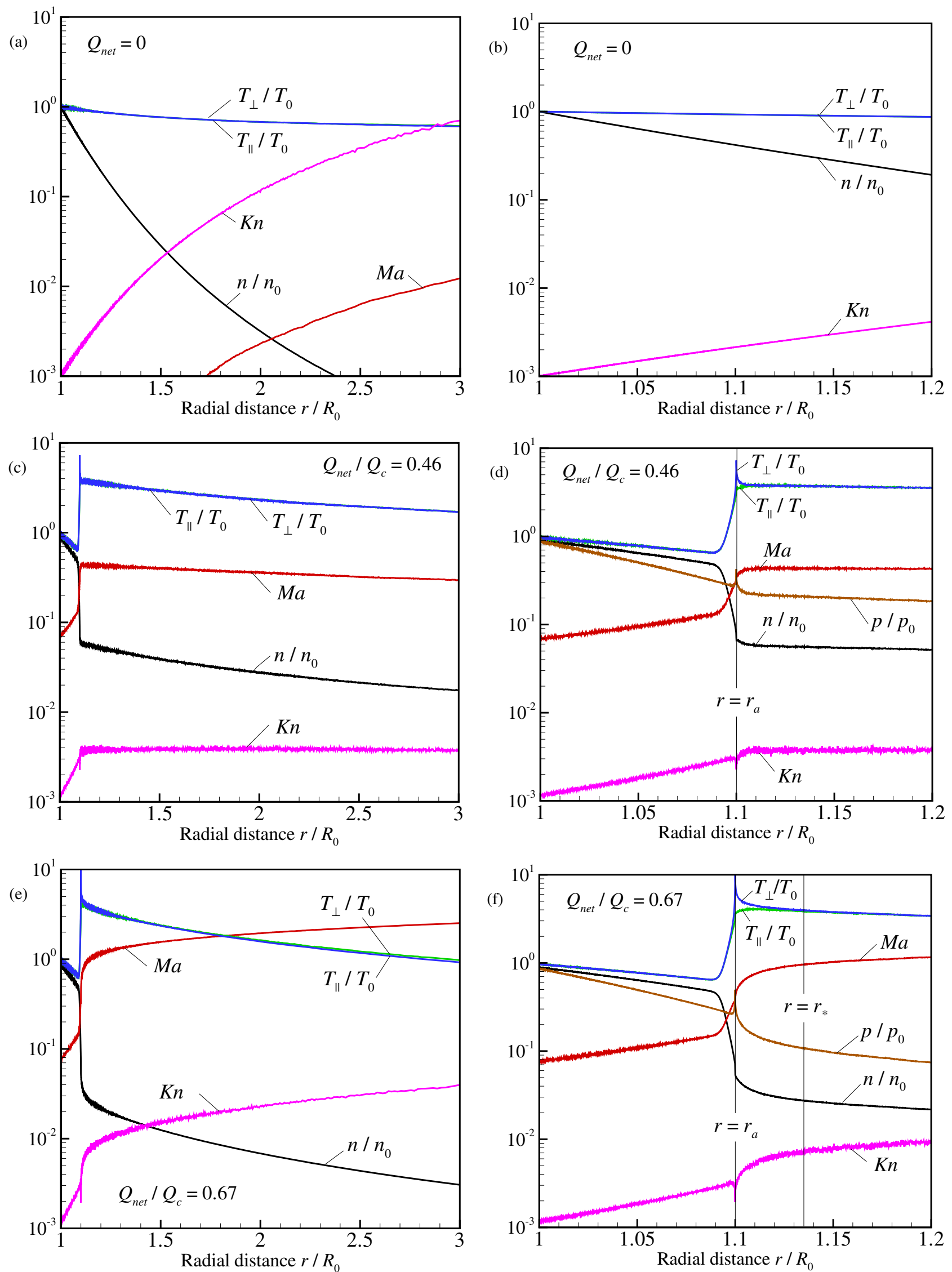

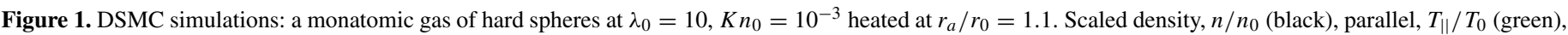

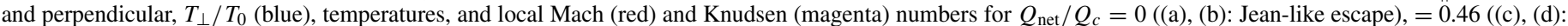
subsonic; $\left.\Phi / \Phi_{E L}=0.87\right)$, and $=0.67\left((\mathrm{e}),(\mathrm{f})\right.$ : transonic at $\left.r_{a} / r_{0}=1.13 ; \Phi / \Phi_{E L}=0.65\right)$. Lines: heated and sonic surfaces.

(A color version of this figure is available in the online journal.) 


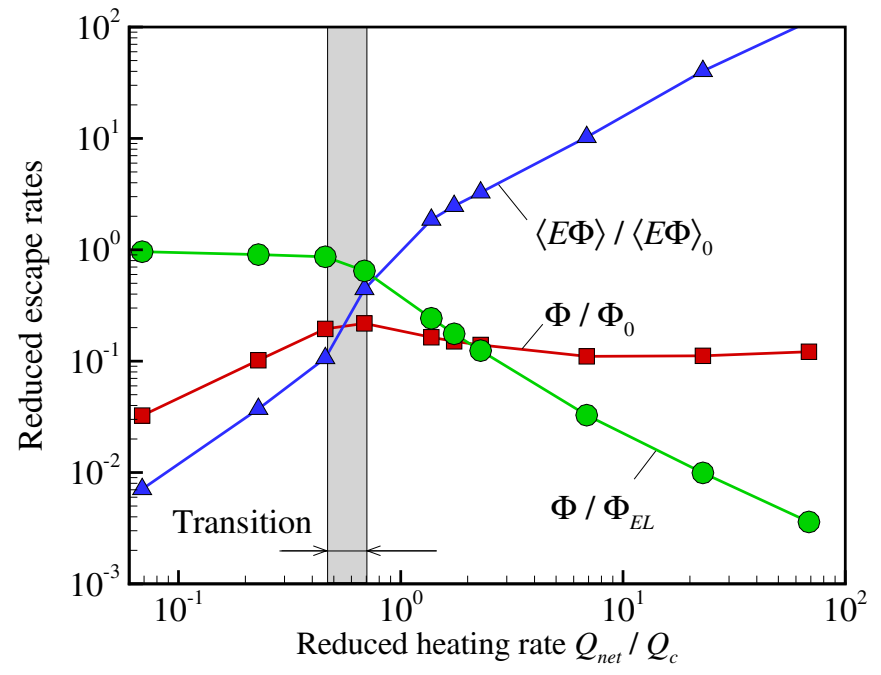

Figure 2. Number $\Phi$ (greencircles, red squares) and energy $\langle E \Phi\rangle$ (blue triangles) escape rates vs. $Q_{\text {net }} / Q_{c}$ calculated as for Figure 1: scaled to $\Phi_{E L}$ from Equation (5), $\Phi_{0}$ from Equation (3a) evaluated at $r_{0}$ instead of at $r_{x}$, and the energy flux of molecules across $r_{0},\langle E \Phi\rangle_{0}=4 \pi r_{0}^{2} n_{0} k T_{0} \sqrt{k T_{0} /(2 \pi m)}$. The rectangle indicates transition from subsonic to supersonic flow below the exobase; for smaller $Q_{\text {net }} / Q_{c}, \Phi \sim \Phi_{E L}$; for larger $Q_{\text {net }} / Q_{c}$, additional heating increases average energy of escaping molecules $\langle E \Phi\rangle / \Phi$.

(A color version of this figure is available in the online journal.)

go sonic in the continuum regime using data from Tian et al. (2008) in Equation (10). It is therefore unlikely that escape from such an atmosphere on a super-Earth in the habitable zone would have a sonic point in the continuum regime.

Lammer et al. (2013) calculated the escape rate from a hydrogen atom thermosphere due to XUV radiation on superEarths observed orbiting close to their star. They used Parker's upper boundary conditions to solve the continuum equations and then decided that blow-off occurred if $\lambda_{x}<3 / 2$ (Öpik 1963). Rather than solving these equations and then deciding whether the sonic or Jeans conditions should have been used, Equation (10) can be used to estimate whether a sonic point might occur in the continuum regime for a given $Q_{\text {net }}$.

For example, using $K n_{m} \sim 1$ and $r_{*} \sim r_{0}$ we find that $Q_{c} \sim 0.6 \times 10^{13} \mathrm{~W}$ and $2.5 \times 10^{13} \mathrm{~W}$ for Kepler11b and $11 \mathrm{c}$, respectively, using data in Table 1 of Lammer et al. (2013). These values can be compared to their heating rates obtained using $\varepsilon=0.15: Q_{\text {net }} \sim 1.2$ and $0.3 \times 10^{13} \mathrm{~W}$, respectively. For this $\varepsilon$, Kepler11b has a sonic point $\left(Q_{\text {net }}>Q_{c}\right)$ in the continuum region, whereas Kepler11c does not $\left(Q_{\text {net }}<Q_{c}\right)$. Therefore, $\Phi_{E L}$ in Equation (5) is most applicable to Kepler11c which requires kinetic, not sonic, boundary conditions to obtain for an accurate description of its upper atmosphere. Of course, increasing $\varepsilon$ or reducing the gravitational energy due to the tides can change this.

\section{SUMMARY}

We have used results from DSMC simulations to show that the oft-used energy-limited rate in Equation (5) for an isentropic expansion of a heated upper atmosphere is most reasonable for a subsonic expansion with a large escape rate. The accuracy depends on how well one estimates $Q_{\text {net }}$. Conversely, agreement with the energy-limited escape rate does not imply that sonic boundary conditions are applicable to continuum models of thermal escape. In fact, the simple approximation in Equation (5) becomes worse with increasing $Q_{\text {net }}$ above $Q_{c}$ as seen in Figure 2. Although the size of the escape rate might not be strongly dependent on whether sonic, Jeans, or kinetic boundary conditions are used, the upper atmosphere can be significantly affected as seen in Figure 3. Therefore, past applications of Parker's (1964a, 1964b) model have led to incorrect descriptions of the upper atmosphere when rapid escape occurs (Tucker \& Johnson 2009; Tucker et al. 2012; Erwin et al 2013). Since the upper atmosphere structure affects the interaction of the escaping gas with the ambient plasma and with neighboring bodies, we have given an expression in Equation (10) to

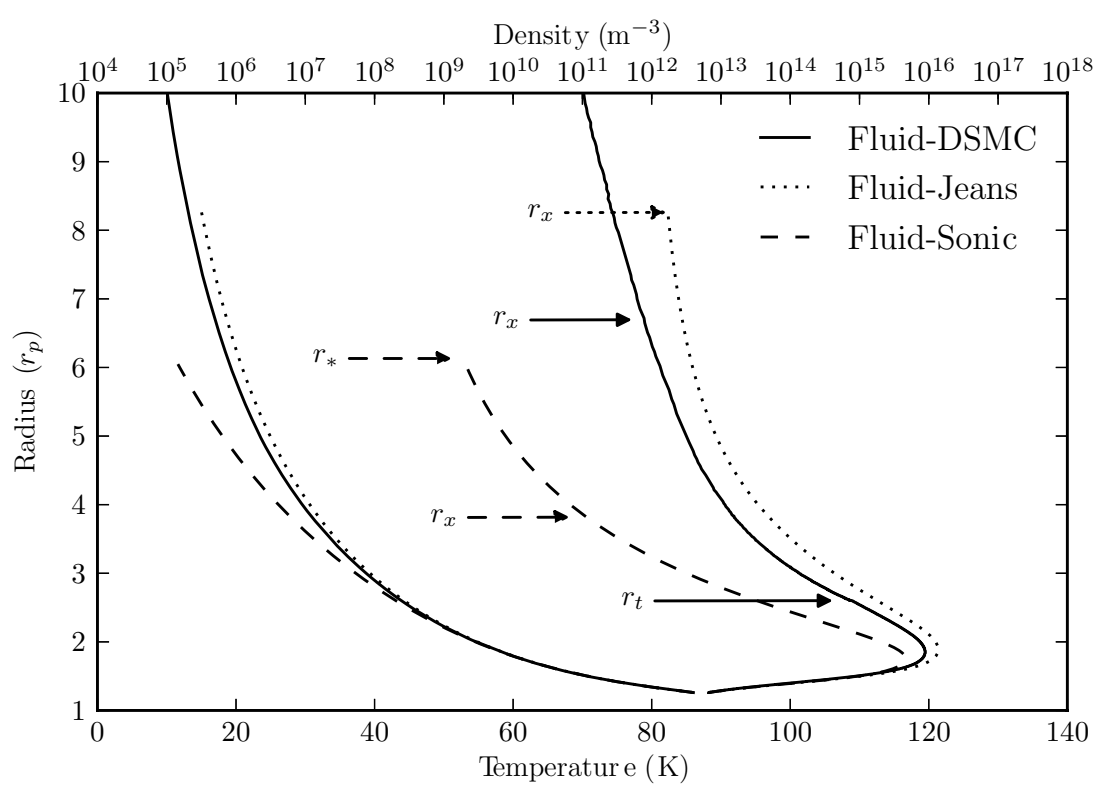

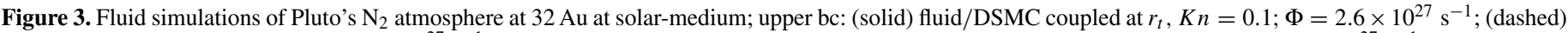

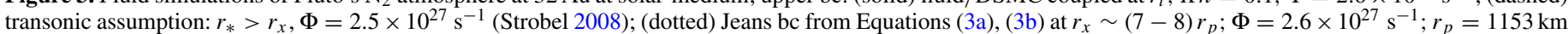

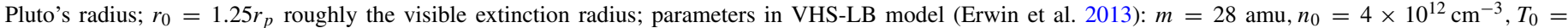
$88.2 \mathrm{~K} ; U\left(r_{0}\right)=2.8 \times 10^{-13} \mathrm{erg} \mathrm{s} ; \lambda_{0}=23 ; \gamma=7 / 5 ; \sigma_{\mathrm{c}} \sim 9 \times 10^{-15} \mathrm{~cm}^{2} ; K n_{0} \sim 10^{-6}$. 
estimate when sonic boundary conditions are likely to be applicable in calculating the escape from and the expansion of the upper atmosphere of a planetary body.

We acknowledge support from NASA's Planetary Atmospheres Program.

\section{APPENDIX}

When $Q_{\text {net }}$ is absorbed in a layer at $r_{a}$ and $u(r)$ is small below $r_{a}$, Equations (1) and (2) can be integrated using $\kappa=\kappa_{0}\left(T / T_{0}\right)^{\omega}$ and assuming zero gas velocity below the heated layer:

$$
\begin{aligned}
Q_{\text {net }}= & E^{+}+\frac{4 \pi \kappa_{0} T_{0} r_{0}}{\omega+1} \frac{r_{a} / r_{0}}{r_{a} / r_{0}-1}\left[\left(\frac{\lambda_{0} r_{0}}{\lambda_{a} r_{a}}\right)^{\omega+1}-1\right] \\
& +4 \pi r_{0}^{2} n_{a} \sqrt{\frac{\gamma}{m \lambda_{a}}} M a_{a}\left[\frac{r_{a}}{r_{0}} U\left(r_{0}\right)\right]^{3 / 2} \frac{r_{0} / r_{a}+1}{2}
\end{aligned}
$$

where $Q_{\text {net }}$ is lost by energy carried off by escaping molecules, $E^{+}$, by downward thermal conduction (second term), and by adiabatic cooling (third term). Simulations having significant escape rates indicate that the last term dominates; assuming $r_{a} / r_{0}-1 \ll 1$ we obtain Equation (9).

\section{REFERENCES}

Bird, G. A. 1994, Molecular Gas Dynamics and the Direct Simulation of Gas Flows (Oxford: Clarendon)

Chamberlain, J. W., \& Hunten, D. M. 1987, Theory of Planetary Atmospheres (New York: Academic)

Erkaev, N. V, Lammer, H., Odert, P., et al. 2012, arXiv:1212.4982

Erwin, J., Tucker, O. J., \& Johnson, R. E. 2013, Icar, submitted

Hunten, D. M. 1982, P\&SS, 30, 773

Hunten, D. M., \& Watson, A. J. 1982, Icar, 51, 665

Johnson, R. E., Combi, M. R., Fox, J. L., et al. 2008, SSRv, 139, 355

Lammer, H., Erkaev, N. V., Odert, P., et al. 2013, MNRAS, 430, 1247

Lammer, H., Odert, P., Leitzinger, M., et al. 2009, A\&A, 506, 399

McNutt, R. L. 1989, GRL, 16, 1225

Murray-Clay, R. A., Chiang, E. I., \& Murray, N. 2009, ApJ, 693, 23

Öpik, E. J. 1963, GJI, 7, 490

Parker, E. N. 1964a, ApJ, 139, 72

Parker, E. N. 1964b, ApJ, 139, 93

Strobel, D. F. 2008, Icar, 193, 612

Tian, F., \& Kasting, J. 2008, JGR, 113, E05008

Tucker, O. J., Erwin, J. T., Deighan, J. I., Volkov, A. N., \& Johnson, R. E. 2012, Icar, 217, 408

Tucker, O. J., \& Johnson, R. E. 2009, P\&SS, 57, 1889

Volkov, A. N., \& Johnson, R. E. 2013, ApJ, 765, 90

Volkov, A. N., Johnson, R. E., Tucker, O. J., \& Erwin, J. T. 2011a, ApJL, 729, L24

Volkov, A. N., Johnson, R. E., Tucker, O. J., \& Erwin, J. T. 2011b, PhFl, 23, 066601

Watson, A. J., Donahue, T. M., \& Walker, J. C. G. 1981, Icar, 48, 150 